\title{
Lewis Base-Catalyzed Ring Opening of Aziridines with Silylated Nucleophiles
}

\section{Supporting Information}

\author{
Satoshi Minakata, * Yuriko Okada, Yoji Oderaotoshi, and Mitsuo Komatsu* \\ Department of Applied Chemistry, Graduate School of Engineering, Osaka University, \\ Yamadaoka 2-1, Suita, Osaka 565-0871, Japan
}

\section{Table of Contents}

General

Typical Procedure for Formation of Aziridines

Spectral Data of Aziridines

General Procedure for Ring Opening of Aziridines 


\section{General.}

Melting points were determined on a Yanagimoto micro melting point apparatus and are uncorrected. IR spectra were obtained on a Jasco FT/IR-410 infrared spectrophotometer. ${ }^{1} \mathrm{H}$ and ${ }^{13} \mathrm{C}-\mathrm{NMR}$ spectra were recorded on a JEOL FT-NMR JNM EX 270 spectrometer ( $\left.{ }^{1} \mathrm{H}-\mathrm{NMR}, 270 \mathrm{MHz} ;{ }^{13} \mathrm{C}-\mathrm{NMR}, 68 \mathrm{MHz}\right)$ using tetramethylsilane as the internal standard. Mass spectra were obtained on a JEOL JMS-DX303HF mass spectrometer. High-resolution mass spectral data were obtained on a JEOL DX-303 mass spectrometer. Elemental analyses were performed at the Analytical Center, Faculty of Engineering, Osaka University. Flash column chromatography (FCC) was performed using silica gel BW-300 (Fuji Silysia Chemical Co.). Preparative gel permeation liquid chromatography (GPLC) was performed on a JAI (Japan Analytical Industry) LC-908 instrument with JAIGEL $1 \mathrm{H}-2 \mathrm{H}$ columns and chloroform as an eluent. Analytical thin layer chromatography was performed using EM reagent $0.25 \mathrm{~mm}$ silica gel $60-\mathrm{F}$ plates. Visualization was accomplished with UV light and ethanolic phosphomolybdic acid followed by heating.

Materials. (a general procedure for preparation of aziridines according to our method ${ }^{1}$ )

The alkenes $(2.0 \mathrm{mmol})$ were added to a solution of iodine $(0.1 \mathrm{mmol}, 10$ mol\%), Chloramine-T $(1.0 \mathrm{mmol})$ in distilled $\mathrm{MeCN}(3.0 \mathrm{~mL})$. The solution was stirred at room temperature under a nitrogen atmosphere. After the addition of $\mathrm{CH}_{2} \mathrm{Cl}_{2}(40 \mathrm{~mL})$ and water $(20 \mathrm{~mL})$, the phases were separated and aqueous phase was extracted with $\mathrm{CH}_{2} \mathrm{Cl}_{2}(20$ $\mathrm{mL})$. The combined organic extracts were washed with water $(20 \mathrm{~mL} \times 3)$ and brine $(20 \mathrm{~mL})$, dried over $\mathrm{K}_{2} \mathrm{CO}_{3}$ and concentrated to give the crude product. Purification by flash column chromatography (silica gel, EtOAc in hexane) gave the aziridines. Further purification was performed by recycling preparative HPLC (GPC column, chloroform), if necessary. 
$\boldsymbol{N}$-(p-Toluenesulfonyl)-2-n-hexylaziridine (1a) $)^{2}$ : Colorless oil; ${ }^{1} \mathrm{H}$ NMR $\left(270 \mathrm{MHz}, \mathrm{CDCl}_{3}\right) \delta=$ 0.86 (t, $3 \mathrm{H}, n-\mathrm{C}_{5} \mathrm{H}_{10} \underline{\mathrm{C}}_{3}, J=7.3 \mathrm{~Hz}$ ), 1.20-1.33 (m, 10H, $\left.n-\mathrm{C}_{5} \underline{\mathrm{H}}_{10} \mathrm{CH}_{3}\right), 2.05$ (d, $1 \mathrm{H}, \mathrm{H}-3, J=$ $4.6 \mathrm{~Hz}), 2.44$ (s, $\left.3 \mathrm{H}, \mathrm{Ar}-\mathrm{C}_{3}\right), 2.62-2.74(\mathrm{~m}, 2 \mathrm{H}, \mathrm{H}-2$ and $\mathrm{H}-3), 7.32(\mathrm{~d}, 2 \mathrm{H}, m-\mathrm{H}, J=8.1 \mathrm{~Hz})$, $7.82(\mathrm{~d}, 2 \mathrm{H}, o-\mathrm{H}, J=8.1 \mathrm{~Hz})$

$\boldsymbol{N}$-(p-Toluenesulfonyl)-2-benzylaziridine (1b) ${ }^{3}$ : White solid; Mp: $94-95{ }^{\circ} \mathrm{C} ;{ }^{1} \mathrm{H}$ NMR $(270$ $\left.\mathrm{MHz}, \mathrm{CDCl}_{3}\right) \delta=2.16(\mathrm{~d}, 1 \mathrm{H}, \mathrm{H}-3, J=4.3 \mathrm{~Hz}), 2.42\left(\mathrm{~s}, 3 \mathrm{H}, \mathrm{Ar}-\underline{\mathrm{C}}_{3}\right), 2.64-2.80\left(\mathrm{~m}, 2 \mathrm{H}, \mathrm{C}_{2}-\mathrm{Ph}\right)$, 2.82-2.99 (m, 2H, H-2 and H-3), 7.01-7.22 (m, 7H, Ph and $m-\mathrm{H}), 7.66(\mathrm{~d}, 2 \mathrm{H}, o-\mathrm{H}, J=8.4 \mathrm{~Hz})$

$\mathrm{N}$-(p-Toluenesulfonyl)-2-sec-butylaziridine (1c) as a mixture of diastereomers: Colorless oil; ${ }^{1} \mathrm{H}$ NMR $\left(270 \mathrm{MHz}, \mathrm{CDCl}_{3}\right) \delta=0.75-1.40\left(\mathrm{~m}, 9 \mathrm{H}, \mathrm{C}_{4} \underline{\mathrm{H}}_{9}\right), 2.09$ (dd, $\left.1 \mathrm{H}, \mathrm{H}-3, J=3.2,13.2 \mathrm{~Hz}\right)$, 2.45-2.67 (m, 5H, H-2, H-3 and Ar-C $\left.\underline{H}_{3}\right), 7.32(\mathrm{~d}, 2 \mathrm{H}, m-\mathrm{H}, J=8.1 \mathrm{~Hz}), 7.81(\mathrm{~d}, 2 \mathrm{H}, o-\mathrm{H}, J=$ $8.1 \mathrm{~Hz}) ;{ }^{13} \mathrm{C}$ NMR $\left(\mathrm{CDCl}_{3}\right) \delta=15.6,21.8,27.2,32.8,36.7,37.1,45.3,128.0,129.5,135.0$, 144.3; MS (CI, isobutane) $\mathrm{m} / z$ (relative intensity, \%) 254 ([M+1 ] , 100); HRMS (CI, isobutane) $m / z$ calcd for $\mathrm{C}_{13} \mathrm{H}_{20} \mathrm{NO}_{2} \mathrm{~S}(\mathrm{M}+\mathrm{H})$ 254.1215, found 254.1228; Anal. Calcd for $\mathrm{C}_{13} \mathrm{H}_{19} \mathrm{NO}_{2} \mathrm{~S}: \mathrm{C}$, $61.63 ; \mathrm{H}, 7.56 ; \mathrm{N}, 5.53$. Found: C, 61.48; H, 7.58; N, 5.44.

$\boldsymbol{N}$-(p-Toluenesulfonyl)-2-phenylaziridine (1d) ${ }^{4}$ : White solid; Mp: $92-93{ }^{\circ} \mathrm{C} ;{ }^{1} \mathrm{H}$ NMR $(270$ $\left.\mathrm{MHz}, \mathrm{CDCl}_{3}\right) \delta=2.38(\mathrm{~d}, 1 \mathrm{H}, \mathrm{H}-3, J=4.3 \mathrm{~Hz}), 2.44$ (s, 3H, Ar-C $\left.\underline{H}_{3}\right), 2.97$ (d, 1H, H-2, $J=$ $7.3 \mathrm{~Hz}$ ), $3.75(\mathrm{dd}, 1 \mathrm{H}, \mathrm{H}-3, J=4.3,7.3 \mathrm{~Hz}), 7.19-7.34(\mathrm{~m}, 7 \mathrm{H}, \mathrm{Ph}$ and $m-\mathrm{H}), 7.85(\mathrm{~d}, 2 \mathrm{H}, o-\mathrm{H}, J$ $=8.4 \mathrm{~Hz})$

$\boldsymbol{N}$-(p-Toluenesulfonyl)-2-hydroxymethylaziridine (1e): Colorless $\quad$ oil; ${ }^{1} \mathrm{H} \quad \mathrm{NMR} \quad(270$ $\left.\mathrm{MHz}, \mathrm{CDCl}_{3}\right) \delta=2.00$ (br s, $1 \mathrm{H}, \mathrm{CH}_{2} \mathrm{OH}, \mathrm{D}_{2} \mathrm{O}$ exchangeable), 2.31 (d, $1 \mathrm{H}, \mathrm{H}-3, J=4.6 \mathrm{~Hz}$ ), 2.45 (s, 3H, Ar- $\left.\underline{\mathrm{H}}_{3}\right), 2.61$ (d, $1 \mathrm{H}, \mathrm{C}_{2} \mathrm{OH}, J=7.0 \mathrm{~Hz}$ ), 2.98-3.06 (m, $\left.1 \mathrm{H}, \mathrm{H}-3\right), 3.50-3.59$ (m, $1 \mathrm{H}, \mathrm{H}-2), 3.80-3.87\left(\mathrm{~m}, 1 \mathrm{H}, \underline{\mathrm{C}}_{2} \mathrm{OH}\right), 7.33(\mathrm{~d}, 2 \mathrm{H}, m-\mathrm{H}, J=8.4 \mathrm{~Hz}), 7.81(\mathrm{~d}, 2 \mathrm{H}, o-\mathrm{H}, J=$ $8.4 \mathrm{~Hz}$ ); MS (CI, methane) $\mathrm{m} / z$ (relative intensity, \%) 228 ([M+1 $\left.]^{+}, 100\right)$; HRMS (CI, methane) $m / z$ Calcd for $\mathrm{C}_{10} \mathrm{H}_{14} \mathrm{NO}_{3} \mathrm{~S}(\mathrm{M}+\mathrm{H}) 228.0695$, found 228.0689 .

1,2-[N-(p-Toluenesulfonyl)imino]cyclopentane $(\mathbf{1 f})^{5}$ : White solid; Mp: $61-62{ }^{\circ} \mathrm{C} ;{ }^{1} \mathrm{H}$ NMR $(270$ $\left.\mathrm{MHz}, \mathrm{CDCl}_{3}\right) \delta=1.37-2.16\left(\mathrm{~m}, 6 \mathrm{H},-\left(\mathrm{C}_{2}\right)_{3}-\right), 2.44\left(\mathrm{~s}, 3 \mathrm{H}, \mathrm{Ar}-\underline{\mathrm{C}}_{3}\right), 3.33(\mathrm{~s}, 2 \mathrm{H}), 7.33(\mathrm{~d}, 2 \mathrm{H}$, $m-\mathrm{H}, J=8.4 \mathrm{~Hz}), 7.79(\mathrm{~d}, 2 \mathrm{H}, o-\mathrm{H}, J=8.4 \mathrm{~Hz})$

1,2-[N-(p-Toluenesulfonyl)imino]cyclohexane (1g) ${ }^{4}$ : White solid; Mp: $55-56{ }^{\circ} \mathrm{C} ; \quad{ }^{1} \mathrm{H}$ NMR 
$\left(270 \mathrm{MHz}, \mathrm{CDCl}_{3}\right) \delta=1.20-1.81\left(\mathrm{~m}, 8 \mathrm{H},-\left(\mathrm{C}_{2}\right)_{4^{-}}\right), 2.44\left(\mathrm{~s}, 3 \mathrm{H}, \mathrm{Ar}^{-\mathrm{CH}_{3}}\right), 2.97(\mathrm{t}, 2 \mathrm{H}, J=1.4 \mathrm{~Hz})$, $7.30(\mathrm{~d}, 2 \mathrm{H}, m-\mathrm{H}, J=8.1 \mathrm{~Hz}), 7.80(\mathrm{~d}, 2 \mathrm{H}, o-\mathrm{H}, J=8.1 \mathrm{~Hz})$

cis- $N$-(p-Toluenesulfonyl)-2-methyl-3- $\boldsymbol{n}$-pentylaziridine (1h) $)^{1}$ : Colorless oil; ${ }^{1} \mathrm{H}$ NMR (270 $\left.\mathrm{MHz}, \quad \mathrm{CDCl}_{3}\right) \delta=0.83\left(\mathrm{t}, \quad 3 \mathrm{H}, \quad n-\mathrm{C}_{4} \mathrm{H}_{8} \mathrm{C}_{3}, \quad J=6.5 \mathrm{~Hz}\right), \quad 1.19-1.41 \quad(\mathrm{~m}$, $11 \mathrm{H}, n-\mathrm{C}_{4} \underline{\mathrm{H}}_{8} \mathrm{CH}_{3}$ and $\left.\mathrm{CHC} \underline{H}_{3}\right), 2.44$ (s, 3H, Ar- $\left.\underline{\mathrm{C}}_{3}\right), 2.68-2.76(\mathrm{~m}, 1 \mathrm{H}, \mathrm{H}-2$ or H-3), 2.87-2.97 $(\mathrm{m}, 1 \mathrm{H}, \mathrm{H}-2$ or $\mathrm{H}-3), 7.33(\mathrm{~d}, 2 \mathrm{H}, m-\mathrm{H}, J=8.2 \mathrm{~Hz}), 7.81(\mathrm{~d}, 2 \mathrm{H}, o-\mathrm{H}, J=8.2 \mathrm{~Hz})$

trans- $N$-(p-Toluenesulfonyl)-2-methyl-3-n-pentylaziridine (1i) ${ }^{1}$ : Colorless oil; ${ }^{1} \mathrm{H}$ NMR $(270$ $\left.\mathrm{MHz}, \quad \mathrm{CDCl}_{3}\right) \delta=0.83\left(\mathrm{t}, 3 \mathrm{H}, \quad n-\mathrm{C}_{4} \mathrm{H}_{8} \mathrm{C}_{3}, \quad J=6.5 \mathrm{~Hz}\right), 1.21-1.58 \quad(\mathrm{~m}$, $11 \mathrm{H}, n-\mathrm{C}_{4} \underline{\mathrm{H}}_{8} \mathrm{CH}_{3}$ and $\left.\mathrm{CHC} \underline{H}_{3}\right), 2.44$ (s, 3H, Ar- $\left.\underline{\mathrm{H}}_{3}\right), 2.67-2.74(\mathrm{~m}, 2 \mathrm{H}, \mathrm{H}-2$ and $\mathrm{H}-3), 7.32$ (d, $2 \mathrm{H}, m-\mathrm{H}, J=8.1 \mathrm{~Hz}), 7.85(\mathrm{~d}, 2 \mathrm{H}, o-\mathrm{H}, J=8.1 \mathrm{~Hz})$ 


\section{General Procedure for Ring Opening of Aziridines.}

To a solution of $N$-tosylaziridine $(0.3 \mathrm{mmol})$ in acetonitrile $(1.2 \mathrm{~mL})$ were added trimethylsilyl compounds $(0.36 \mathrm{mmol})$ and TMEDA $(9 \mu \mathrm{L}, \quad 0.06$ mmol). The mixture was stirred at the temperature indicated in the text and the progress of the reaction was monitored by TLC. After consumption of the starting material, removal of solvent under reduced pressure gave the crude product, which was purified by silica gel column chromatography using $n$-hexane-AcOEt as an eluent to give the corresponding product.

$\mathrm{N}$-(2-Cyanooctyl)-4-methylbenzenesulfonamide (2a) ${ }^{6}$ : Colorless oil (76.7 mg, 83\%); IR (neat, $\left.\mathrm{cm}^{-1}\right) 2251(\mathrm{CN}) ;{ }^{1} \mathrm{H}$ NMR $\left(270 \mathrm{MHz}, \mathrm{CDCl}_{3}\right) \delta=0.84\left(\mathrm{t}, 3 \mathrm{H}, n-\mathrm{C}_{5} \mathrm{H}_{10} \mathrm{C}_{3}, J=6.8 \mathrm{~Hz}\right)$, 1.07-1.60 (m, $\left.10 \mathrm{H}, n-\mathrm{C}_{5} \underline{\mathrm{H}}_{10} \mathrm{CH}_{3}\right), 2.44$ (s, $\left.3 \mathrm{H}, \mathrm{Ar}_{-} \underline{\mathrm{C}}_{3}\right), 2.50-2.68\left(\mathrm{~m}, 2 \mathrm{H}, \underline{\mathrm{C}}_{2} \mathrm{CN}\right), 3.38-3.46(\mathrm{~m}$,

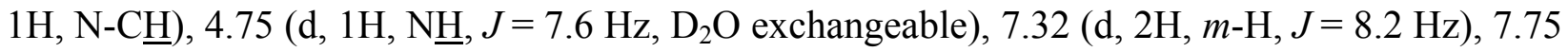
$(\mathrm{d}, 2 \mathrm{H}, o-\mathrm{H}, J=8.2 \mathrm{~Hz}) ;{ }^{13} \mathrm{C} \mathrm{NMR}\left(\mathrm{CDCl}_{3}\right) \delta=14.1,21.6,22.5,25.1,25.3,28.5,31.5,33.9,50.0$, 116.7(CN), 127.0, 129.8, 136.9, 143.8; MS (CI, methane) $\mathrm{m} / \mathrm{z}$ (relative intensity, \%) $309\left([\mathrm{M}+1]^{+}\right.$, 61), 268 ([M-CH $2 \mathrm{CN}]^{+}, 100$ ); HRMS (CI, methane) $m / z$ Calcd for $\mathrm{C}_{16} \mathrm{H}_{25} \mathrm{~N}_{2} \mathrm{O}_{2} \mathrm{~S}(\mathrm{M}+\mathrm{H})$ 309.1637, found 309.1646; Anal. Calcd for $\mathrm{C}_{16} \mathrm{H}_{24} \mathrm{~N}_{2} \mathrm{O}_{2} \mathrm{~S}$ : C, 62.30; H, 7.84; N, 9.08. Found: C, $61.89 ; \mathrm{H}, 7.52 ; \mathrm{N}, 8.92$.

$\mathrm{N}$-(2-Cyano-1-benzylethyl)-4-methylbenzenesulfonamide (2b): White solid (79.1 $\mathrm{mg}, 84 \%)$; Mp: 102-103 ${ }^{\circ} \mathrm{C}$; IR (neat, $\left.\mathrm{cm}^{-1}\right) 2251(\mathrm{CN}) ;{ }^{1} \mathrm{H}$ NMR $\left(270 \mathrm{MHz}, \mathrm{CDCl}_{3}\right) \delta=2.42(\mathrm{~s}$, $\left.3 \mathrm{H}, \mathrm{Ar}-\underline{\mathrm{C}}_{3}\right), 2.51-2.58$ (dd, $1 \mathrm{H}, \mathrm{C}_{2} \mathrm{Ph}, J=3.8,16.7 \mathrm{~Hz}$ ), 2.62-2.70 (dd, $1 \mathrm{H}, \mathrm{C}_{2} \mathrm{Ph}, J=6.5$, $16.7 \mathrm{~Hz}$ ), 2.73-2.81 (dd, $1 \mathrm{H}, \underline{\mathrm{C}}_{2} \mathrm{CN}, J=7.8,13.9 \mathrm{~Hz}$ ), 2.86-2.93 (dd, $1 \mathrm{H}, \underline{\mathrm{C}}_{2} \mathrm{CN}, J=6.8$,

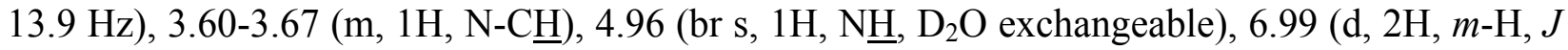
$=8.4 \mathrm{~Hz}), 7.18-7.22(\mathrm{~m}, 5 \mathrm{H}, \mathrm{Ph}), 7.54(\mathrm{~d}, 2 \mathrm{H}, o-\mathrm{H}, J=8.4 \mathrm{~Hz}) ;{ }^{13} \mathrm{C} \mathrm{NMR}\left(\mathrm{CDCl}_{3}\right) \delta=21.6,24.2$, 39.8, 51.4, $116.7(\mathrm{CN}), 126.8,127.2,128.3,128.9,129.7,134.9,136.2,143.7$; MS (CI, methane) $\mathrm{m} / z$ (relative intensity, \%) $315\left([\mathrm{M}+1]^{+}, 100\right), 274\left(\left[\mathrm{M}-\mathrm{CH}_{2} \mathrm{CN}\right]^{+}, 100\right)$; HRMS (CI, methane) $m / z$ Calcd for $\mathrm{C}_{17} \mathrm{H}_{19} \mathrm{~N}_{2} \mathrm{O}_{2} \mathrm{~S}(\mathrm{M}+\mathrm{H}) 315.1168$, found 315.1154. Anal. Calcd for $\mathrm{C}_{17} \mathrm{H}_{18} \mathrm{~N}_{2} \mathrm{O}_{2} \mathrm{~S}: \mathrm{C}$, 64.94; H, 5.77; N, 8.91. Found: C, 64.65; H, 5.76; N, 8.73. 
$\mathrm{N}$-(1-Cyanomethyl-2-methylbutyl)-4-methylbenzenesulfonamide (2c) as a mixture of diastereomers: Colorless oil (78.1 mg, 93\%); IR (neat, $\left.\mathrm{cm}^{-1}\right) 2251(\mathrm{CN}) ;{ }^{1} \mathrm{H}$ NMR (270 $\left.\mathrm{MHz}, \mathrm{CDCl}_{3}\right) \delta=0.72-1.67\left(\mathrm{~m}, 9 \mathrm{H}, \mathrm{C}_{4} \underline{\mathrm{H}}_{9}\right), 2.44$ (s, 3H, $\left.\mathrm{Ar}-\underline{\mathrm{C}}_{3}\right), 2.55-2.63\left(\mathrm{~m}, 2 \mathrm{H}, \underline{\mathrm{C}}_{2} \mathrm{CN}\right)$,

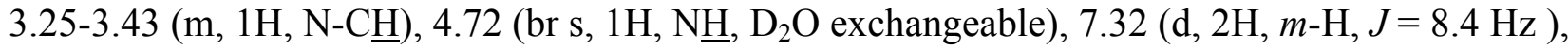
$7.78(\mathrm{~d}, 2 \mathrm{H}, o-\mathrm{H}, J=8.4 \mathrm{~Hz}) ;{ }^{13} \mathrm{C} \mathrm{NMR}\left(\mathrm{CDCl}_{3}\right) \delta=15.3,21.7,22.3,24.5,37.3,37.6,53.4$, 116.7(CN), 127.0, 129.8, 136.9, 144.0; MS (CI, methane) $\mathrm{m} / z$ (relative intensity, \%) $281\left([\mathrm{M}+1]^{+}\right.$, 37), 240 ([M-CH$\left.\left.{ }_{2} \mathrm{CN}\right]^{+}, 100\right)$; HRMS (CI, methane) $m / z$ Calcd for $\mathrm{C}_{14} \mathrm{H}_{21} \mathrm{~N}_{2} \mathrm{O}_{2} \mathrm{~S}(\mathrm{M}+\mathrm{H}$ ) 281.1324, found 281.1318; Anal. Calcd for $\mathrm{C}_{14} \mathrm{H}_{20} \mathrm{~N}_{2} \mathrm{O}_{2} \mathrm{~S}$ : C, 59.97; H, 7.19; N, 9.99. Found: C, 59.69; H, 6.94; N, 9.86.

$N$-(2-Cyano-1-phenylethyl)-4-methylbenzenesulfonamide (2d) ${ }^{6}$ : White solid (42.3 $\mathrm{mg}, 47 \%$ ); Mp: 120-121 ${ }^{\circ} \mathrm{C}$; IR (neat, $\left.\mathrm{cm}^{-1}\right) 2249(\mathrm{CN}) ;{ }^{1} \mathrm{H}$ NMR $\left(270 \mathrm{MHz}, \mathrm{CDCl}_{3}\right) \delta=2.42(\mathrm{~s}$, $\left.3 \mathrm{H}, \mathrm{Ar}-\mathrm{C}_{3}\right), 2.93$ (ddd, 2H, $\left.\underline{\mathrm{H}}_{2} \mathrm{CN}, J=5.4,7.0,16.7 \mathrm{~Hz}\right), 4.54-4.59$ (m, 1H, N-C $\underline{\mathrm{H}}$ ), 5.09 (d, $1 \mathrm{H}, \mathrm{N} \underline{\mathrm{H}}, J=6.8 \mathrm{~Hz}, \mathrm{D}_{2} \mathrm{O}$ exchangeable), $7.12(\mathrm{~d}, 2 \mathrm{H}, m-\mathrm{H}, J=8.2 \mathrm{~Hz}), 7.24-7.31(\mathrm{~m}, 5 \mathrm{H}, \mathrm{Ph})$, $7.68(\mathrm{~d}, 2 \mathrm{H}, o-\mathrm{H}, J=8.2 \mathrm{~Hz}) ;{ }^{13} \mathrm{C} \mathrm{NMR}\left(\mathrm{CDCl}_{3}\right) \delta=21.5,26.3,54.1,116.4(\mathrm{CN}), 126.2,127.1$, 128.9, 129.2, 129.8, 136.4, 137.1, 144.0; MS (CI, methane) $m / z$ (relative intensity, \%) 301 $\left([\mathrm{M}+1]^{+}, 100\right), 260\left(\left[\mathrm{M}-\mathrm{CH}_{2} \mathrm{CN}\right]^{+}, 43\right)$; HRMS (CI, methane) $m / z$ Calcd for $\mathrm{C}_{16} \mathrm{H}_{17} \mathrm{~N}_{2} \mathrm{O}_{2} \mathrm{~S}(\mathrm{M}+\mathrm{H})$ 301.1011, found 301.1008. Anal. Calcd for $\mathrm{C}_{16} \mathrm{H}_{16} \mathrm{~N}_{2} \mathrm{O}_{2} \mathrm{~S}$ : C, 63.98; H, 5.37; N, 9.33. Found: C, 63.70; H, 5.32; N, 9.21.

N-(2-Cyano-1-hydroxymethylethyl)-4-methylbenzenesulfonamide (2e): Colorless oil (30.5 mg, 40\%); IR (neat, $\mathrm{cm}^{-1}$ ) $2252(\mathrm{CN}) ;{ }^{1} \mathrm{H}$ NMR (270 MHz, $\left.\mathrm{CDCl}_{3}\right) \delta=2.44\left(\mathrm{~s}, 3 \mathrm{H}, \mathrm{Ar}^{-\mathrm{CH}_{3}}\right)$, 2.62-2.66 (m, 3H, $\underline{\mathrm{C}}_{2} \mathrm{CN}$ and $\mathrm{O} \underline{\mathrm{H}}, \mathrm{D}_{2} \mathrm{O}$ exchangeable), 3.61-3.67 (m, 3H, N-C태 and $\left.\underline{\mathrm{C}}_{2} \mathrm{OH}\right)$, $5.66\left(\mathrm{~d}, 1 \mathrm{H}, \mathrm{N} \underline{\mathrm{H}}, J=7.6 \mathrm{~Hz}, \mathrm{D}_{2} \mathrm{O}\right.$ exchangeable), $7.32(\mathrm{~d}, 2 \mathrm{H}, m-\mathrm{H}, J=8.4 \mathrm{~Hz}), 7.78(\mathrm{~d}, 2 \mathrm{H}$, $o-\mathrm{H}, J=8.4 \mathrm{~Hz}) ;{ }^{13} \mathrm{C} \mathrm{NMR}\left(\mathrm{CDCl}_{3}\right) \delta=21.0,21.7,51.1,62.6,116.7(\mathrm{CN}), 127.0,129.9,136.6$, 144.1; MS (CI, methane) $m / z$ (relative intensity, \%) $255\left([\mathrm{M}+1]^{+}, 100\right), 223\left(\left[\mathrm{M}-\mathrm{CH}{ }_{2} \mathrm{OH}\right]^{+}\right.$, 25); HRMS (CI, methane) $m / z$ Calcd for $\mathrm{C}_{11} \mathrm{H}_{15} \mathrm{~N}_{2} \mathrm{O}_{3} \mathrm{~S}(\mathrm{M}+\mathrm{H})$ 255.0804, found 255.0806. 
N-[2-Cyano-(1-trimethylsilyloxymethyl)ethyl]-4-methylbenzenesulfonamide: Colorless oil (47.0 mg, 48\%); IR (neat, $\mathrm{cm}^{-1}$ ) $2254(\mathrm{CN}) ;{ }^{1} \mathrm{H}$ NMR (270 MHz, $\mathrm{CDCl}_{3}$ ) $\delta=0.087$ (s, 9H, TMS), $2.44\left(\mathrm{~s}, 3 \mathrm{H}, \mathrm{Ar}-\mathrm{C}_{3}\right), 2.61-2.64\left(\mathrm{~m}, 2 \mathrm{H}, \underline{\mathrm{CH}}_{2} \mathrm{CN}\right), 3.45-3.63\left(\mathrm{~m}, 3 \mathrm{H}, \mathrm{N}-\mathrm{C} \underline{\mathrm{H}}\right.$ and $\left.\mathrm{C}_{2} \mathrm{OTMS}\right), 5.10$ $\left(\mathrm{d}, 1 \mathrm{H}, \mathrm{N} \underline{\mathrm{H}}, J=7.8 \mathrm{~Hz}, \mathrm{D}_{2} \mathrm{O}\right.$ exchangeable), $7.32(\mathrm{~d}, 2 \mathrm{H}, m-\mathrm{H}, J=8.4 \mathrm{~Hz}), 7.78(\mathrm{~d}, 2 \mathrm{H}, o-\mathrm{H}, J=$ $8.4 \mathrm{~Hz}) ;{ }^{13} \mathrm{C} \mathrm{NMR}\left(\mathrm{CDCl}_{3}\right) \delta=-0.7,21.1,21.6,51.1,62.2,116.6(\mathrm{CN}), 127.0,129.9,136.9$, 143.9; MS (CI, methane) $m / z$ (relative intensity, \%) 327 ([M+1] $\left.]^{+}, 100\right)$; HRMS (CI, methane) $m / z$ Calcd for $\mathrm{C}_{14} \mathrm{H}_{23} \mathrm{~N}_{2} \mathrm{O}_{3} \mathrm{SSi}(\mathrm{M}+\mathrm{H}) 327.1200$, found 327.1206 .

N-(2-Cyanocyclopentyl)-4-methylbenzenesulfonamide (2f) ${ }^{6}$ : White solid (55.4 mg, 70\%); Mp: 107-108 ${ }^{\circ} \mathrm{C}$; IR (neat, $\left.\mathrm{cm}^{-1}\right) 2243(\mathrm{CN}) ;{ }^{1} \mathrm{H}$ NMR $\left(270 \mathrm{MHz}, \mathrm{CDCl}_{3}\right) \delta=1.44-2.11(\mathrm{~m}, 6 \mathrm{H}$, $\left.-\left(\mathrm{CH}_{2}\right)_{3}-\right), 2.43$ (s, 3H, Ar- $\left.\underline{\mathrm{H}}_{3}\right), 2.83$ (ddd, 1H, $\left.\mathrm{CHCN}, J=5.9,7.3,10.5 \mathrm{~Hz}\right), 3.74-3.78(\mathrm{~m}, 1 \mathrm{H}$,

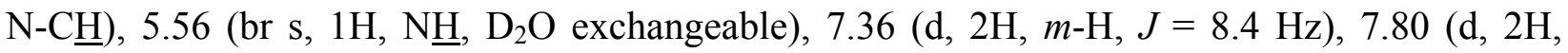
$o-\mathrm{H}, J=8.4 \mathrm{~Hz}) ;{ }^{13} \mathrm{C} \mathrm{NMR}\left(\mathrm{CDCl}_{3}\right) \delta=21.7,22.7,29.0,32.7,35.7,58.6,121.0(\mathrm{CN}), 127.1$, 129.9, 136.3, 144.0; MS (CI, methane) $\mathrm{m} / z$ (relative intensity, \%) $265\left([\mathrm{M}+1]^{+}, 100\right), 172$ ([M-CH $3 \mathrm{Ar}]^{+}$, 22); HRMS (CI, methane) $m / z$ Calcd for $\mathrm{C}_{13} \mathrm{H}_{17} \mathrm{~N}_{2} \mathrm{O}_{2} \mathrm{~S}(\mathrm{M}+\mathrm{H})$ 265.1011, found 265.1004; Anal. Calcd for $\mathrm{C}_{13} \mathrm{H}_{16} \mathrm{~N}_{2} \mathrm{O}_{2} \mathrm{~S}$ : C, 59.07; H, 6.10; N, 10.60. Found: C, 58.80; H, 6.08; N, 10.43 .

N-(2-Cyanocyclohexyl)-4-methylbenzenesulfonamide (2g) ${ }^{6}$ : White solid (80.9 mg, 97\%); Mp: 115-116 ${ }^{\circ} \mathrm{C}$; IR (neat, $\left.\mathrm{cm}^{-1}\right) 2245(\mathrm{CN}) ;{ }^{1} \mathrm{H}$ NMR $\left(270 \mathrm{MHz}, \mathrm{CDCl}_{3}\right) \delta=1.24-2.05(\mathrm{~m}, 8 \mathrm{H}$, $\left.-\left(\mathrm{CH}_{2}\right)_{4}-\right), 2.44$ (s, 3H, Ar- $\left.\underline{H}_{3}\right), 2.65$ (ddd, 1H, $\left.\underline{\mathrm{HCN}}, J=3.8,8.1,8.9 \mathrm{~Hz}\right), 3.35-3.39$ (m, 1H,

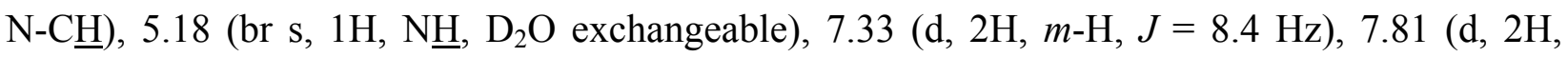
$o-\mathrm{H}, J=8.4 \mathrm{~Hz}) ;{ }^{13} \mathrm{C} \mathrm{NMR}\left(\mathrm{CDCl}_{3}\right) \delta=21.7,22.6,23.0,27.3,31.4,34.6,52.7,120.2(\mathrm{CN})$, 127.1, 129.7, 137.0, 143.8; MS (CI, methane) $\mathrm{m} / z$ (relative intensity, \%) $279\left([\mathrm{M}+1]^{+}, 100\right), 125$ ([M-CH $\left.\mathrm{CHSO}_{2}\right]^{+}$, 29); HRMS (CI, methane) $m / z$ Calcd for $\mathrm{C}_{14} \mathrm{H}_{19} \mathrm{~N}_{2} \mathrm{O}_{2} \mathrm{~S}(\mathrm{M}+\mathrm{H}) 279.1167$, found 279.1159 .

\section{N-(2-Cyano-2-methylheptyl)-4-methylbenzenesulfonamide (2h, syn form):}

Colorless oil (55.7 mg, 60\%); IR (neat, $\mathrm{cm}^{-1}$ ) $2241(\mathrm{CN}) ;{ }^{1} \mathrm{H}$ NMR $\left(270 \mathrm{MHz}, \mathrm{CDCl}_{3}\right) \delta=$ 0.75-1.72 (m, 14H, n- $\mathrm{C}_{5} \underline{\mathrm{H}}_{11}$ and $\left.\mathrm{CHC}_{3}\right), 2.43\left(\mathrm{~s}, 3 \mathrm{H}, \mathrm{Ar}_{-} \underline{\mathrm{H}}_{3}\right), 2.87-2.96(\mathrm{~m}, 1 \mathrm{H}, \mathrm{C} \underline{\mathrm{HCN}})$, 3.28-3.38 (m, 1H, N-C프), 4.83 (br s, $1 \mathrm{H}, \mathrm{N} \underline{\mathrm{H}}, \mathrm{D}_{2} \mathrm{O}$ exchangeable), 7.32 (d, 2H, $m-\mathrm{H}, J=8.2 \mathrm{~Hz}$ ), $7.75(\mathrm{~d}, 2 \mathrm{H}, o-\mathrm{H}, J=8.2 \mathrm{~Hz}) ;{ }^{13} \mathrm{C} \mathrm{NMR}\left(\mathrm{CDCl}_{3}\right) \delta=13.7,13.8,21.5,22.2,25.3,30.9,31.0,32.8$, 
55.3, 120.3(CN), 126.8, 129.6, 137.3, 143.6; HRMS (CI, methane) $\mathrm{m} / z$ Calcd for $\mathrm{C}_{16} \mathrm{H}_{25} \mathrm{~N}_{2} \mathrm{O}_{2} \mathrm{~S}$ $(\mathrm{M}+\mathrm{H})$ 309.1637, found 309.1630.

\section{$\mathrm{N}$-(2-Cyano-1-methylheptyl)-4-methylbenzenesulfonamide (3h, syn form):}

Colorless oil (27.8 mg, 30\%); IR (neat, $\left.\mathrm{cm}^{-1}\right) 2241(\mathrm{CN}) ;{ }^{1} \mathrm{H}$ NMR $\left(270 \mathrm{MHz}, \mathrm{CDCl}_{3}\right) \delta=$ 0.75-1.72 (m, $14 \mathrm{H}, n-\mathrm{C}_{5} \underline{\mathrm{H}}_{11}$ and $\left.\mathrm{CHC}_{3}\right), 2.43\left(\mathrm{~s}, 3 \mathrm{H}, \operatorname{Ar}-\underline{\mathrm{C}}_{3}\right), 2.56-2.61(\mathrm{~m}, 1 \mathrm{H}, \mathrm{C} \underline{H C N})$,

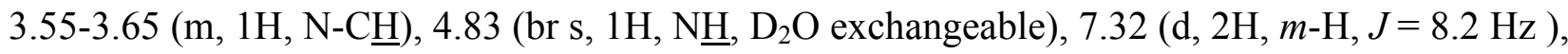
$7.75(\mathrm{~d}, 2 \mathrm{H}, o-\mathrm{H}, J=8.2 \mathrm{~Hz}) ;{ }^{13} \mathrm{C} \mathrm{NMR}\left(\mathrm{CDCl}_{3}\right) \delta=13.9,19.7,22.3,25.3,26.9,28.3,31.1,39.0$, 53.4, 119.5(CN), 126.8, 129.6, 137.3, 143.6; HRMS (CI, methane) $\mathrm{m} / z$ Calcd for $\mathrm{C}_{16} \mathrm{H}_{25} \mathrm{~N}_{2} \mathrm{O}_{2} \mathrm{~S}$ $(\mathrm{M}+\mathrm{H}) 309.1637$, found 309.1630.

\section{$\mathrm{N}$-(2-Cyano-2-methylheputyl)-4-methylbenzenesulfonamide (2i, anti form):}

Colorless oil (59.1 mg, 64\%); IR (neat, $\left.\mathrm{cm}^{-1}\right) 2243(\mathrm{CN}) ;{ }^{1} \mathrm{H}$ NMR $\left(270 \mathrm{MHz}, \mathrm{CDCl}_{3}\right) \delta=$ 0.73-1.82 (m, $14 \mathrm{H}, n-\mathrm{C}_{5} \underline{\mathrm{H}}_{11}$ and $\left.\mathrm{CHC}_{3}\right), 2.43\left(\mathrm{~s}, 3 \mathrm{H}, \mathrm{Ar}-\underline{\mathrm{C}}_{3}\right), 2.98-3.08(\mathrm{~m}, 1 \mathrm{H}, \mathrm{C} \underline{\mathrm{HCN}})$, 3.12-3.22 (m, 1H, N-C $\underline{\mathrm{H}}$ ), 5.23 (br s, $1 \mathrm{H}, \mathrm{N} \underline{\mathrm{H}}, \mathrm{D}_{2} \mathrm{O}$ exchangeable), 7.32 (d, $2 \mathrm{H}, m-\mathrm{H}, J=8.1 \mathrm{~Hz}$ ), $7.77(\mathrm{~d}, 2 \mathrm{H}, o-\mathrm{H}, J=8.1 \mathrm{~Hz}) ;{ }^{13} \mathrm{C} \mathrm{NMR}\left(\mathrm{CDCl}_{3}\right) \delta=13.9,15.2,21.6,22.4,24.9,29.5,31.0,33.0$, 55.7, 120.4(CN), 126.8, 129.7, 137.3, 143.7; HRMS (CI, methane) $m / z$ Calcd for $\mathrm{C}_{16} \mathrm{H}_{25} \mathrm{~N}_{2} \mathrm{O}_{2} \mathrm{~S}$ $(\mathrm{M}+\mathrm{H}) 309.1637$, found 309.1631.

\section{N-(2-Cyano-1-methylheptyl)-4-methylbenzenesulfonamide (3i, anti form):}

Colorless oil (14.8 mg, 16\%); IR (neat, $\left.\mathrm{cm}^{-1}\right) 2241(\mathrm{CN}) ;{ }^{1} \mathrm{H}$ NMR $\left(270 \mathrm{MHz}, \mathrm{CDCl}_{3}\right) \delta=$ 0.73-1.82 (m, $14 \mathrm{H}, n-\mathrm{C}_{5} \underline{\mathrm{H}}_{11}$ and $\left.\mathrm{CHC}_{3}\right), 2.43\left(\mathrm{~s}, 3 \mathrm{H}, \mathrm{Ar}-\underline{\mathrm{C}}_{3}\right), 2.80-2.82(\mathrm{~m}, 1 \mathrm{H}, \mathrm{C} \underline{\mathrm{HCN}})$,

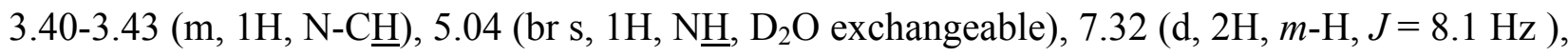
$7.77(\mathrm{~d}, 2 \mathrm{H}, o-\mathrm{H}, J=8.1 \mathrm{~Hz}) ;{ }^{13} \mathrm{C} \mathrm{NMR}\left(\mathrm{CDCl}_{3}\right) \delta=14.0,17.4,22.4,25.4,27.0,30.2,31.1,39.8$, 53.5, 119.5(CN), 126.9, 129.8, 137.4, 143.6; HRMS (CI, methane) $\mathrm{m} / z$ Calcd for $\mathrm{C}_{16} \mathrm{H}_{25} \mathrm{~N}_{2} \mathrm{O}_{2} \mathrm{~S}$ $(\mathrm{M}+\mathrm{H})$ 309.1637, found 309.1631.

$\boldsymbol{N}$-(2-Azidooctyl)-4-methylbenzenesulfonamide (4a) ${ }^{6}$ : Colorless oil (87.5 mg, 90\%); IR (neat, $\left.\mathrm{cm}^{-1}\right) 2104\left(\mathrm{~N}_{3}\right) ;{ }^{1} \mathrm{H}$ NMR $\left(270 \mathrm{MHz}, \mathrm{CDCl}_{3}\right) \delta=0.87\left(\mathrm{t}, 3 \mathrm{H}, n-\mathrm{C}_{5} \mathrm{H}_{10} \mathrm{C}_{3}, J=6.5 \mathrm{~Hz}\right), 1.11-1.49$ (m, $\left.10 \mathrm{H}, n-\mathrm{C}_{5} \underline{\mathrm{H}}_{10} \mathrm{CH}_{3}\right), 2.43$ (s, 3H, Ar- $\left.\underline{\mathrm{C}}_{3}\right), 3.26-3.36\left(\mathrm{~m}, 3 \mathrm{H}, \mathrm{C}_{2} \mathrm{~N}_{3}\right.$ and $\left.\mathrm{N}-\mathrm{C} \underline{\mathrm{H}}\right), 4.57(\mathrm{~d}, 1 \mathrm{H}$, $\mathrm{N} \underline{\mathrm{H}}, J=7.6 \mathrm{~Hz}, \mathrm{D}_{2} \mathrm{O}$ exchangeable), $7.31(\mathrm{~d}, 2 \mathrm{H}, m-\mathrm{H}, J=8.2 \mathrm{~Hz}), 7.76(\mathrm{~d}, 2 \mathrm{H}, o-\mathrm{H}, J=8.2 \mathrm{~Hz})$; ${ }^{13} \mathrm{C} \mathrm{NMR}\left(\mathrm{CDCl}_{3}\right) \delta=14.1,21.6,22.5,25.3,28.7,31.3,32.5,53.2,54.9,126.9,129.6,137.5$, 
143.4; MS (CI, isobutane) $\mathrm{m} / z$ (relative intensity, \%) $325\left([\mathrm{M}+1]^{+}, 58\right), 268\left(\left[\mathrm{M}_{-} \mathrm{CH}_{2} \mathrm{~N}_{3}\right]^{+}\right.$, 100); HRMS (CI, isobutane) $m / z$ Calcd for $\mathrm{C}_{15} \mathrm{H}_{25} \mathrm{~N}_{4} \mathrm{O}_{2} \mathrm{~S}(\mathrm{M}+\mathrm{H}) 3251698$, found 325.1696 .

$\mathrm{N}$-(2-Bromooctyl)-4-methylbenzenesulfonamide (5a): Colorless oil (95.8 mg, 88\%); ${ }^{1} \mathrm{H}$ NMR $\left(270 \mathrm{MHz}, \mathrm{CDCl}_{3}\right) \delta=0.85\left(\mathrm{t}, 3 \mathrm{H}, n-\mathrm{C}_{5} \mathrm{H}_{10} \mathrm{C}_{3}, J=6.8 \mathrm{~Hz}\right), 1.13-1.61\left(\mathrm{~m}, 10 \mathrm{H}, n-\mathrm{C}_{5} \underline{\mathrm{H}}_{10} \mathrm{CH}_{3}\right)$, 2.43 (s, $\left.3 \mathrm{H}, \mathrm{Ar}-\mathrm{C}_{3}\right), 3.30-3.43\left(\mathrm{~m}, 3 \mathrm{H}, \mathrm{C}_{2} \mathrm{Br}\right.$ and $\left.\mathrm{N}-\mathrm{C} \underline{\mathrm{H}}\right), 4.77$ (d, $1 \mathrm{H}, \mathrm{N} \underline{\mathrm{H}}, J=8.4 \mathrm{~Hz}, \mathrm{D}_{2} \mathrm{O}$ exchangeable), $7.31(\mathrm{~d}, 2 \mathrm{H}, m-\mathrm{H}, J=8.6 \mathrm{~Hz}), 7.81(\mathrm{~d}, 2 \mathrm{H}, o-\mathrm{H}, J=8.6 \mathrm{~Hz}) ;{ }^{13} \mathrm{C} \mathrm{NMR}\left(\mathrm{CDCl}_{3}\right) \delta$ $=14.1,21.6,22.5,25.3,28.7,31.6,33.4,38.3,53.2,126.9,129.6,137.6,143.5$; MS (CI, isobutane) $\mathrm{m} / \mathrm{z}$ (relative intensity, \%) $364\left([\mathrm{M}+1]^{+}, 100\right), 362\left([\mathrm{M}+1]^{+}, 97\right), 282\left([\mathrm{M}-\mathrm{Br}]^{+}\right.$, 93); HRMS (CI, isobutane) $m / z$ Calcd for $\mathrm{C}_{15} \mathrm{H}_{25} \mathrm{~B}_{\mathrm{r}} \mathrm{NO}_{2} \mathrm{~S}(\mathrm{M}+\mathrm{H}) 362.0789$, found 362.0786 .

$\mathrm{N}$-(2-Iodooctyl)-4-methylbenzenesulfonamide (6a): Colorless oil (119.0 mg, 97\%); ${ }^{1} \mathrm{H}$ NMR $\left(270 \mathrm{MHz}, \mathrm{CDCl}_{3}\right) \delta=0.85\left(\mathrm{t}, 3 \mathrm{H}, n-\mathrm{C}_{5} \mathrm{H}_{10} \mathrm{C}_{3}, J=6.5 \mathrm{~Hz}\right), 1.13-1.60\left(\mathrm{~m}, 10 \mathrm{H}, n-\mathrm{C}_{5} \underline{\mathrm{H}}_{10} \mathrm{CH}_{3}\right)$, 2.43 (s, 3H, Ar- $\underline{\mathrm{CH}}_{3}$ ), 2.88-2.99 (m, 1H, N-C $\underline{\mathrm{H}}$ ), 3.18 (dd, $1 \mathrm{H}, \mathrm{C}_{2} \mathrm{I}, J=5.4,10.4 \mathrm{~Hz}$ ), 3.25 (dd, $1 \mathrm{H}, \mathrm{C}_{2} \mathrm{I}, J=7.3,10.4 \mathrm{~Hz}$ ), $4.65\left(\mathrm{~d}, 1 \mathrm{H}, \mathrm{N} \underline{\mathrm{H}}, J=8.6 \mathrm{~Hz}, \mathrm{D}_{2} \mathrm{O}\right.$ exchangeable), 7.31 (d, 2H, $m-\mathrm{H}, J=8.3 \mathrm{~Hz}), 7.77(\mathrm{~d}, 2 \mathrm{H}, o-\mathrm{H}, J=8.3 \mathrm{~Hz}) ;{ }^{13} \mathrm{C} \mathrm{NMR}\left(\mathrm{CDCl}_{3}\right) \delta=14.1,15.0,21.6,22.5$, $25.2,28.7, \quad 31.6,35.6, \quad 52.5,126.9,129.6,137.7,143.5$; MS (CI, isobutane) $\mathrm{m} / \mathrm{z}$ (relative intensity, \%) $410\left([\mathrm{M}+1]^{+}, 100\right), 282$ ([M-I $\left.]^{+}, 87\right)$; HRMS (CI, methane) $m / z$ Calcd for $\mathrm{C}_{15} \mathrm{H}_{25} \mathrm{INO}_{2} \mathrm{~S}(\mathrm{M}+\mathrm{H}) 410.0651$, found 410.0638 .

$N$-(2-Azido-1-benzylethyl)-4-methylbenzenesulfonamide (4b): Colorless oil (87.1 $\mathrm{mg}$, 88\%); IR (neat, $\left.\mathrm{cm}^{-1}\right) 2104\left(\mathrm{~N}_{3}\right) ;{ }^{1} \mathrm{H}$ NMR $\left(270 \mathrm{MHz}, \mathrm{CDCl}_{3}\right) \delta=2.42$ (s, $\left.3 \mathrm{H}, \mathrm{Ar}-\mathrm{C}_{3}\right)$, 2.64-2.81 (dd, $2 \mathrm{H}, \mathrm{C}_{2} \mathrm{Ph}, J=7.0,11.0 \mathrm{~Hz}$ ), 3.26-3.38 (dd, $2 \mathrm{H}, \mathrm{C}_{2} \mathrm{~N}_{3}, J=4.3,12.4 \mathrm{~Hz}$ ), 3.46-3.58 (m, 1H, N-C $\underline{\mathrm{H}}), 5.02\left(\mathrm{~d}, 1 \mathrm{H}, \mathrm{N} \underline{\mathrm{H}}, J=7.6 \mathrm{~Hz}, \mathrm{D}_{2} \mathrm{O}\right.$ exchangeable), $6.98(\mathrm{~d}, 2 \mathrm{H}, m-\mathrm{H}, J$ $=8.4 \mathrm{~Hz}), 7.13-7.31(\mathrm{~m}, 5 \mathrm{H}, \mathrm{Ph}), 7.60(\mathrm{~d}, 2 \mathrm{H}, o-\mathrm{H}, J=8.4 \mathrm{~Hz}) ;{ }^{13} \mathrm{C} \mathrm{NMR}\left(\mathrm{CDCl}_{3}\right) \delta=21.6$, 38.5, 53.7, 54.3, 126.8, 127.2, 128.6, 129.0, 129.6, 136.0, 136.8, 143.3; MS (CI, isobutane) $\mathrm{m} / \mathrm{z}$

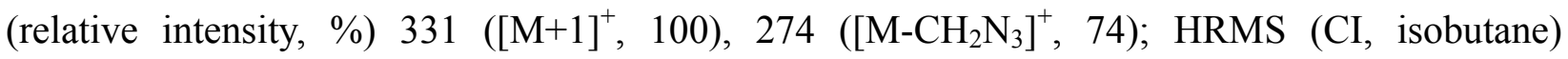
$m / z$ Calcd for $\mathrm{C}_{16} \mathrm{H}_{19} \mathrm{~N}_{4} \mathrm{O}_{2} \mathrm{~S}(\mathrm{M}+\mathrm{H}) 331.1229$, found 331.1221. 
N-(2-Bromo-1-benzylethyl)-4-methylbenzenesulfonamide (5b): Colorless oil (96.9 mg, 88\%); ${ }^{1} \mathrm{H}$ NMR $\left(270 \mathrm{MHz}, \mathrm{CDCl}_{3}\right) \delta=2.41$ (s, 3H, $\left.\mathrm{Ar}-\underline{\mathrm{C}}_{3}\right), 2.63-2.99$ (m, 3H, $\underline{\mathrm{C}}_{2} \mathrm{Ph}$ and $\mathrm{C}_{2} \mathrm{Br}$ ),

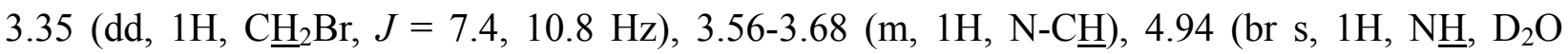
exchangeable), $7.04(\mathrm{~d}, 2 \mathrm{H}, m-\mathrm{H}, J=8.4 \mathrm{~Hz}), 7.13-7.31(\mathrm{~m}, 5 \mathrm{H}, \mathrm{Ph}), 7.65(\mathrm{~d}, 2 \mathrm{H}, o-\mathrm{H}, J=$ 8.4 Hz); ${ }^{13} \mathrm{C} \mathrm{NMR}\left(\mathrm{CDCl}_{3}\right) \delta=21.6,37.0,39.2,54.6,126.8,127.7,128.3,128.5,129.6,136.0$, 137.0, 143.6; MS (CI, isobutane) $m / z$ (relative intensity, \%) $370\left([\mathrm{M}+1]^{+}, 73\right), 368\left([\mathrm{M}+1]^{+}, 71\right)$, 288 ([M-Br $\left.]^{+}, 100\right)$; HRMS (CI, isobutane) $\mathrm{m} / z$ Calcd for $\mathrm{C}_{16} \mathrm{H}_{19} \mathrm{BrNO}_{2} \mathrm{~S}(\mathrm{M}+\mathrm{H}) 368.0320$, found 368.0312 .

$\boldsymbol{N}$-(2-Iodo-1-benzylethyl)-4-methylbenzenesulfonamide (6b): Colorless oil (123.3 mg, 99\%); ${ }^{1} \mathrm{H}$ NMR $\left(270 \mathrm{MHz}, \mathrm{CDCl}_{3}\right) \delta=2.41$ (s, 3H, Ar- $\left.\underline{\mathrm{H}}_{3}\right), 2.68-2.95$ (m, 2H, $\left.\underline{\mathrm{H}}_{2} \mathrm{Ph}\right), 3.14-3.27$ (m, $3 \mathrm{H}, \underline{\mathrm{C}}_{2} \mathrm{I}$ and $\left.\mathrm{N}-\mathrm{C} \underline{\mathrm{H}}\right), 4.90\left(\mathrm{~d}, 1 \mathrm{H}, \mathrm{N} \underline{\mathrm{H}}, J=7.8 \mathrm{~Hz}, \quad \mathrm{D}_{2} \mathrm{O}\right.$ exchangeable $), 7.04(\mathrm{~d}, 2 \mathrm{H}, m-\mathrm{H}, J=$ $8.1 \mathrm{~Hz}), 7.15-7.23(\mathrm{~m}, 5 \mathrm{H}, \mathrm{Ph}), 7.67(\mathrm{~d}, 2 \mathrm{H}, o-\mathrm{H}, J=8.1 \mathrm{~Hz}) ;{ }^{13} \mathrm{C} \mathrm{NMR}\left(\mathrm{CDCl}_{3}\right) \delta=21.6,34.0$, 36.2, 54.1, 126.8, 126.9, 128.6, 129.0, 129.6, 136.0, 137.0, 143.4 ; MS (CI, isobutane) $\mathrm{m} / \mathrm{z}$ (relative intensity, \%) $416\left([\mathrm{M}+1]^{+}, 40\right), 288\left([\mathrm{M}-\mathrm{I}]^{+}, 100\right) ;$ HRMS (CI, isobutane) $m / z$ Calcd for $\mathrm{C}_{16} \mathrm{H}_{19} \mathrm{INO}_{2} \mathrm{~S}(\mathrm{M}+\mathrm{H}) 416.0182$, found 416.0184 .

$\boldsymbol{N}$-(2-Azidocyclopentyl)-4-methylbenzenesulfonamide (4f) ${ }^{6}$ : Colorless oil (81.5 mg, 97\%); IR (neat, $\left.\mathrm{cm}^{-1}\right) 2102\left(\mathrm{~N}_{3}\right) ;{ }^{1} \mathrm{H}$ NMR $\left.\left(270 \mathrm{MHz} \mathrm{CDCl}_{3}\right) \delta=1.23-2.05\left(\mathrm{~m}, 6 \mathrm{H},-\left(\mathrm{C}_{2}\right)_{3}\right)^{-}\right), 2.44(\mathrm{~s}$, $\left.3 \mathrm{H}, \mathrm{Ar}-\underline{\mathrm{C}}_{3}\right), 3.38$ (ddd, $\left.1 \mathrm{H}, \underline{\mathrm{CHN}}_{3}, J=6.5,8.9,12.2 \mathrm{~Hz}\right), \quad 3.71-3.74$ (m, 1H, N-C $\left.\underline{\mathrm{H}}\right), 5.18$ (br s, $1 \mathrm{H}, \mathrm{N} \underline{\mathrm{H}}, \mathrm{D}_{2} \mathrm{O}$ exchangeable), $7.32(\mathrm{~d}, 2 \mathrm{H}, m-\mathrm{H}, J=8.1 \mathrm{~Hz}), 7.79(\mathrm{~d}, 2 \mathrm{H}, o-\mathrm{H}, J=8.1 \mathrm{~Hz}) ;{ }^{13} \mathrm{C}$ NMR $\left(\mathrm{CDCl}_{3}\right) \delta=20.9,21.6,29.0,31.0,59.8,67.0,127.1,129.7,136.9$, 143.6; MS (CI, isobutane) $\mathrm{m} / \mathrm{z}$ (relative intensity, \%) $281\left([\mathrm{M}+1]^{+}, 18\right), 253\left(\left[\mathrm{M}-\mathrm{N}_{2}\right]^{+}, 42\right), 238\left(\left[\mathrm{M}-\mathrm{N}_{3}\right]^{+}\right.$, 10); HRMS (CI, methane) $\mathrm{m} / z$ Calcd for $\mathrm{C}_{12} \mathrm{H}_{17} \mathrm{~N}_{4} \mathrm{O}_{2} \mathrm{~S}(\mathrm{M}+\mathrm{H}) 281.1073$, found 281.1062.

N-(2-Azidocyclohexyl)-4-methylbenzenesulfonamide (4g) ${ }^{6}$ : Colorless oil (86.4 mg, 98\%); IR (neat, $\left.\mathrm{cm}^{-1}\right) 2100\left(\mathrm{~N}_{3}\right) ;{ }^{1} \mathrm{H}$ NMR $\left.\left(270 \mathrm{MHz}, \mathrm{CDCl}_{3}\right) \delta=1.23-2.04\left(\mathrm{~m}, 8 \mathrm{H},-\left(\mathrm{C}_{2}\right)_{4}\right)^{-}\right), 2.43(\mathrm{~s}$, $\left.3 \mathrm{H}, \operatorname{Ar}-\underline{\mathrm{C}}_{3}\right), 2.94-3.10\left(\mathrm{~m}, 2 \mathrm{H}, \mathrm{C}^{-} \mathrm{N}_{3}\right.$ and $\left.\mathrm{N}-\mathrm{C} \underline{\mathrm{H}}\right), 4.75\left(\mathrm{~d}, 1 \mathrm{H}, \mathrm{N} \underline{\mathrm{H}}, J=6.2 \mathrm{~Hz}, \mathrm{D}_{2} \mathrm{O}\right.$ exchangeable), $7.31(\mathrm{~d}, 2 \mathrm{H}, m-\mathrm{H}, J=8.1 \mathrm{~Hz}), 7.80(\mathrm{~d}, 2 \mathrm{H}, o-\mathrm{H}, J=8.1 \mathrm{~Hz}) ;{ }^{13} \mathrm{C} \mathrm{NMR}\left(\mathrm{CDCl}_{3}\right) \delta$ $=21.5,23.6,23.8,30.1,32.5,56.7,63.5,126.9,129.5,137.2,143.3$; MS (CI, isobutane) $\mathrm{m} / \mathrm{z}$

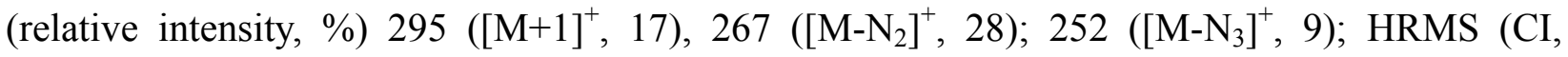
methane) $m / z$ Calcd for $\mathrm{C}_{13} \mathrm{H}_{19} \mathrm{~N}_{4} \mathrm{O}_{2} \mathrm{~S}(\mathrm{M}+\mathrm{H})$ 295.1228, found 295.1230. 


\section{References}

1. Ando, T.; Kano, D.; Minakata, S.; Ryu, I.; Komatsu, M. Tetrahedron 1998, 54, 13485-13494.

2. Toshima, A.; Abe, H.; Hirosawa, C.; Tamao, K. J. Chem. Soc. Perkin Trans. 1, 1994, $3465-3471$.

3. Alonso, D. A.; Andersson, P. G. J. Org. Chem. 1998, 63, 9455-9461.

4. Evans, D. A.; Faul, M. M.; Bilodeau, M. T. J. Am. Chem. Soc. 1994, 116, 2742-2753.

5. Jeong, J. U.; Tao, B.; Sagasser, I.; Henniges, H.; Sharpless, K. B. J. Am. Chem. Soc. 1998, 120, 6844-6845.

6. Wu, J.; Hou, X.-L.; Dai, L.-X. J. Org. Chem. 2000, 65, 1344-1348. 


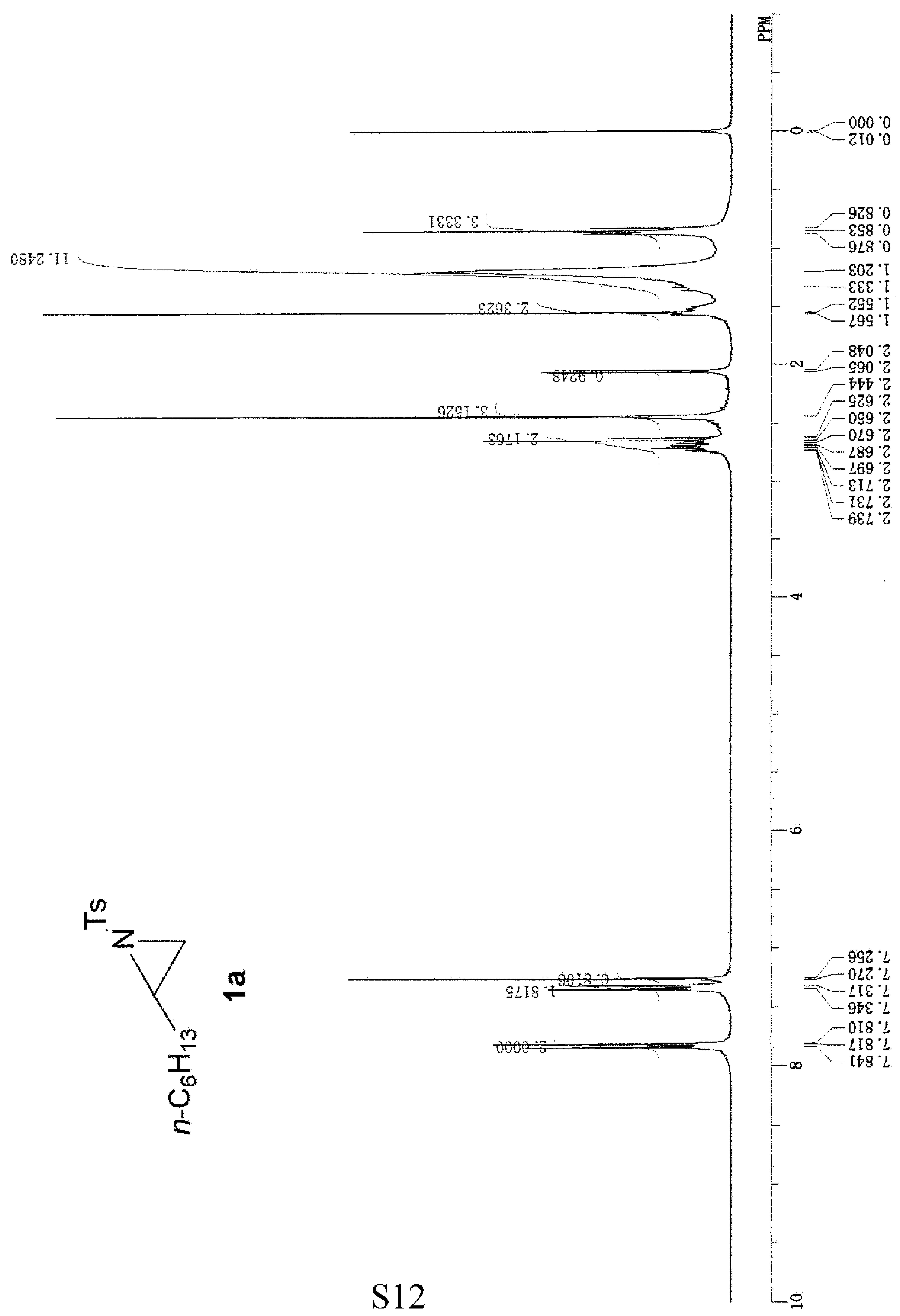




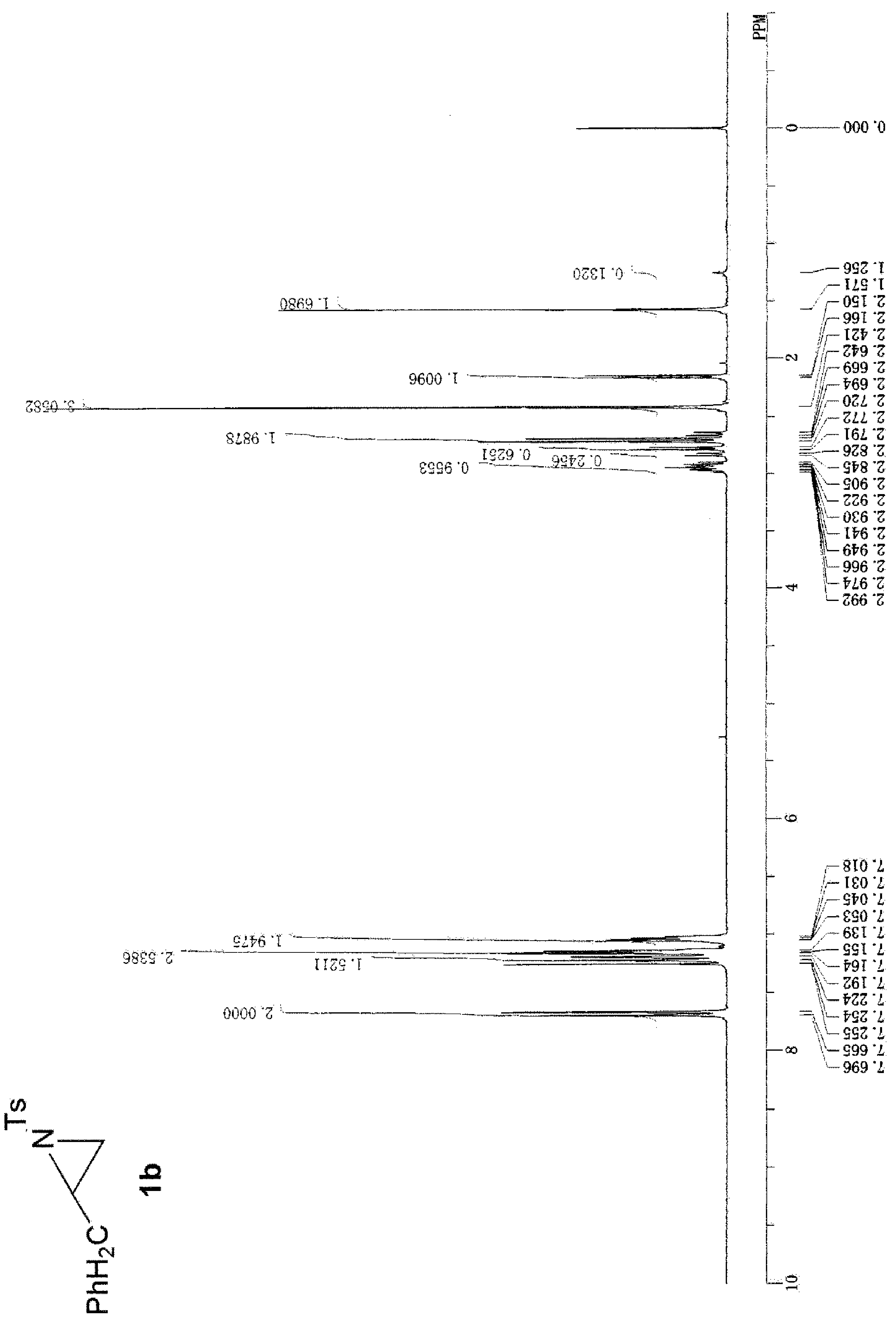




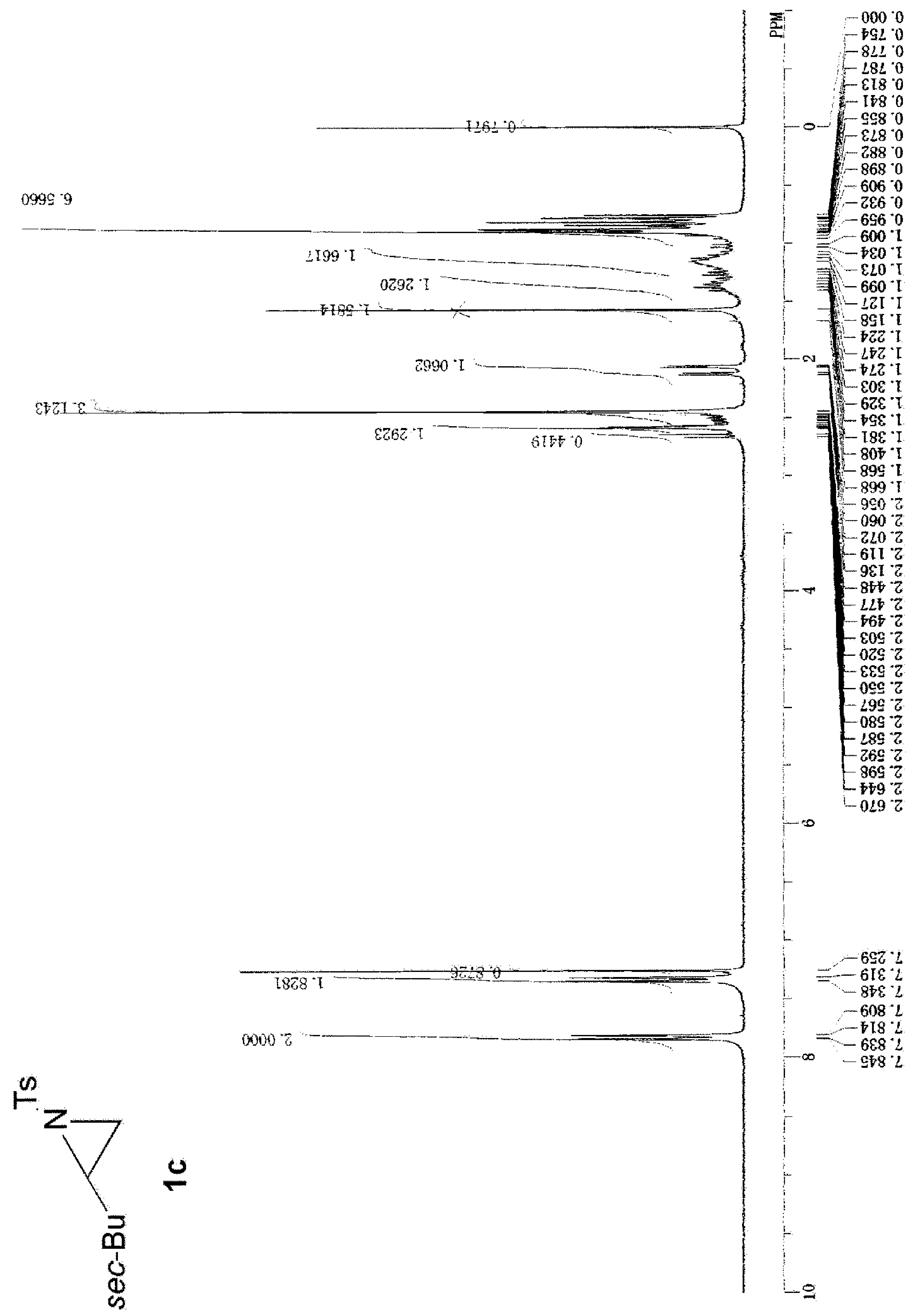




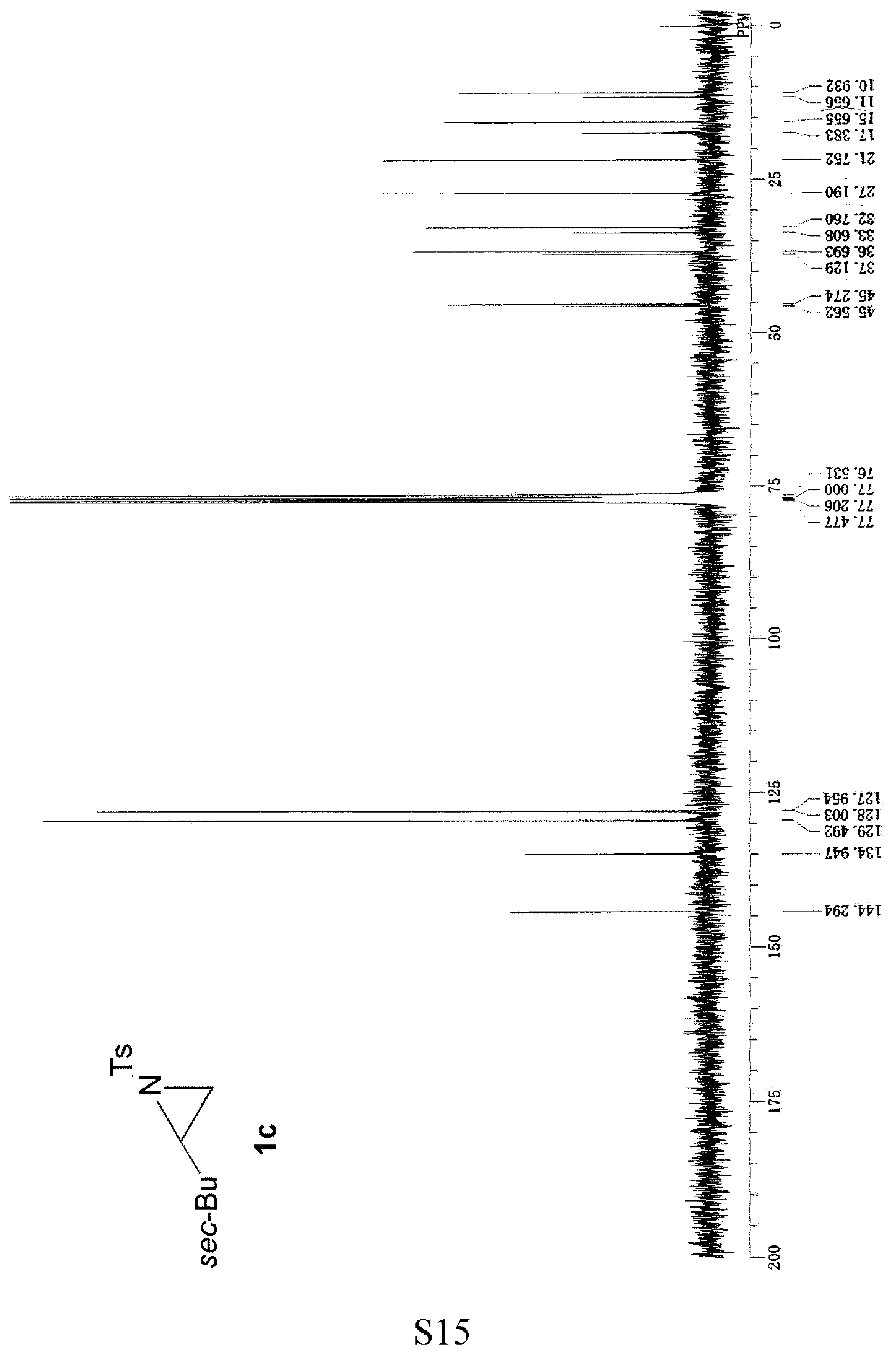




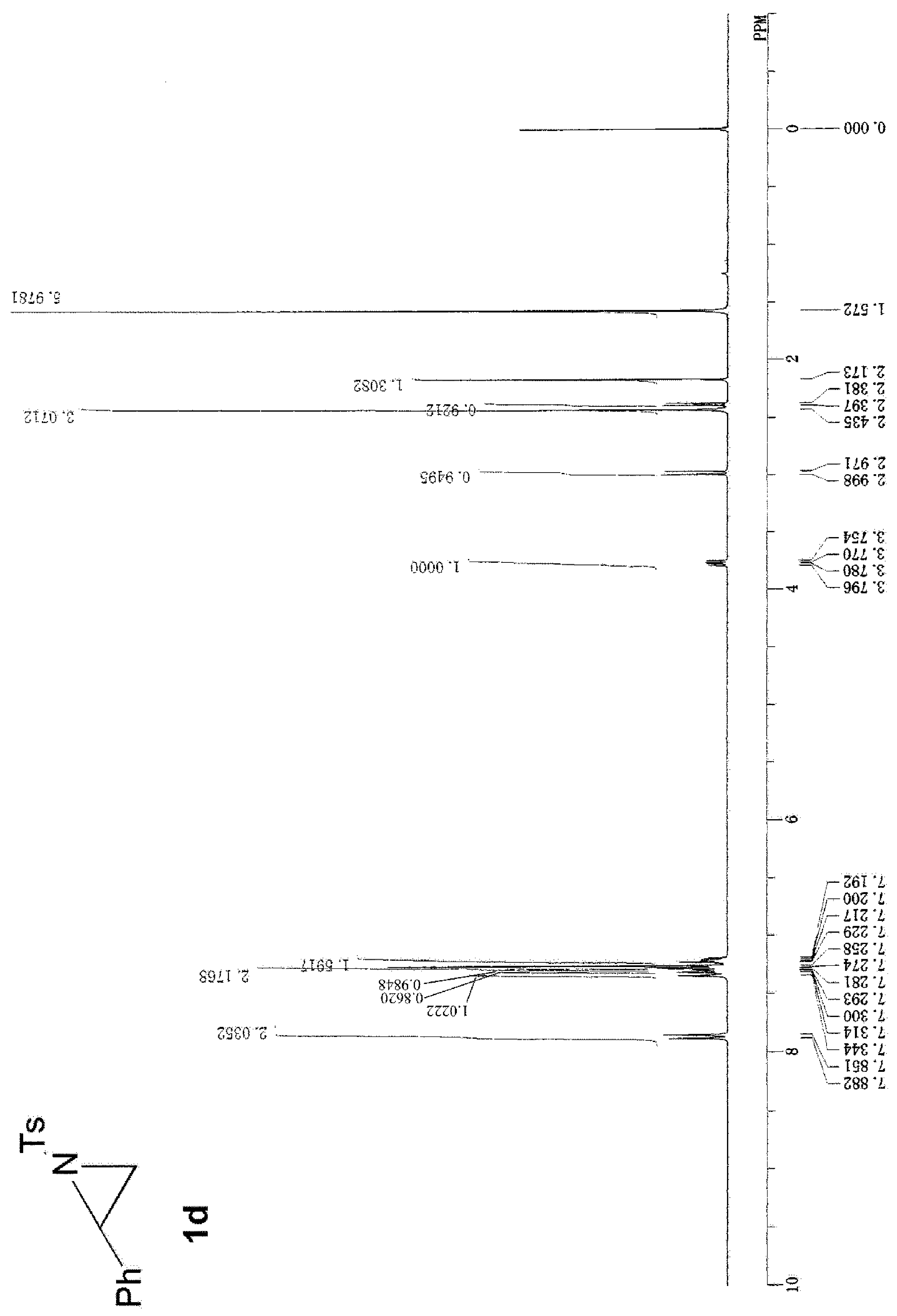




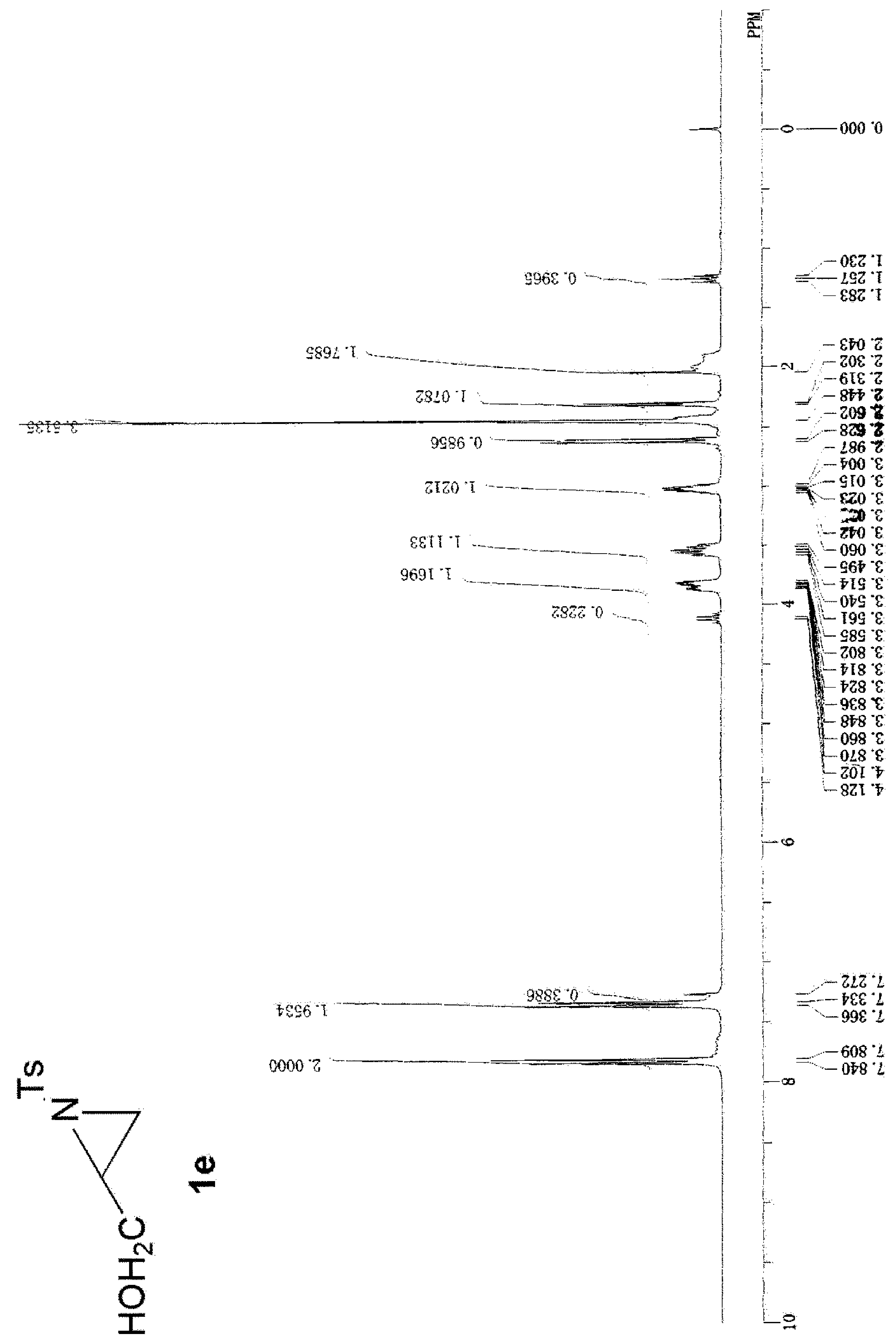




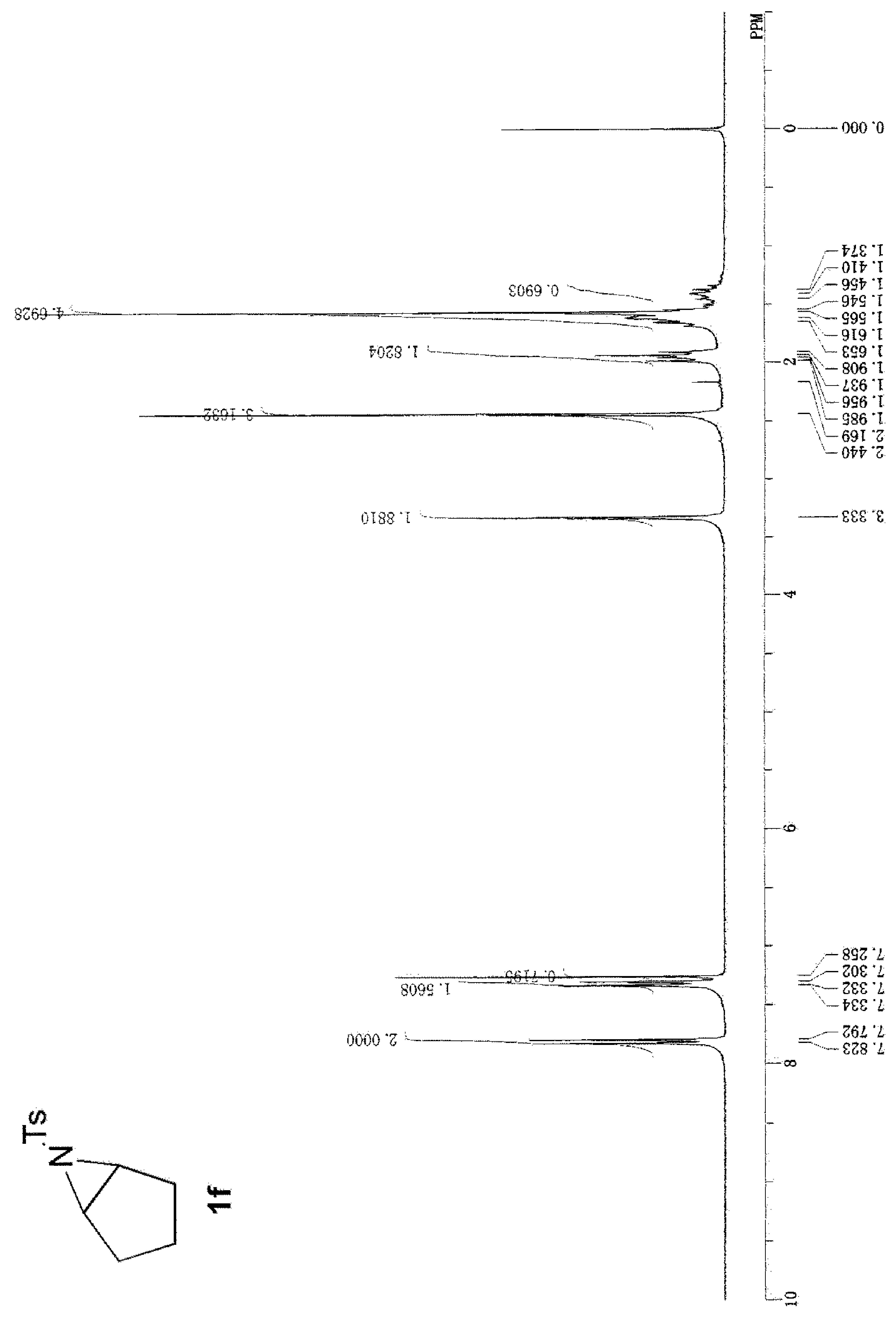

S18 


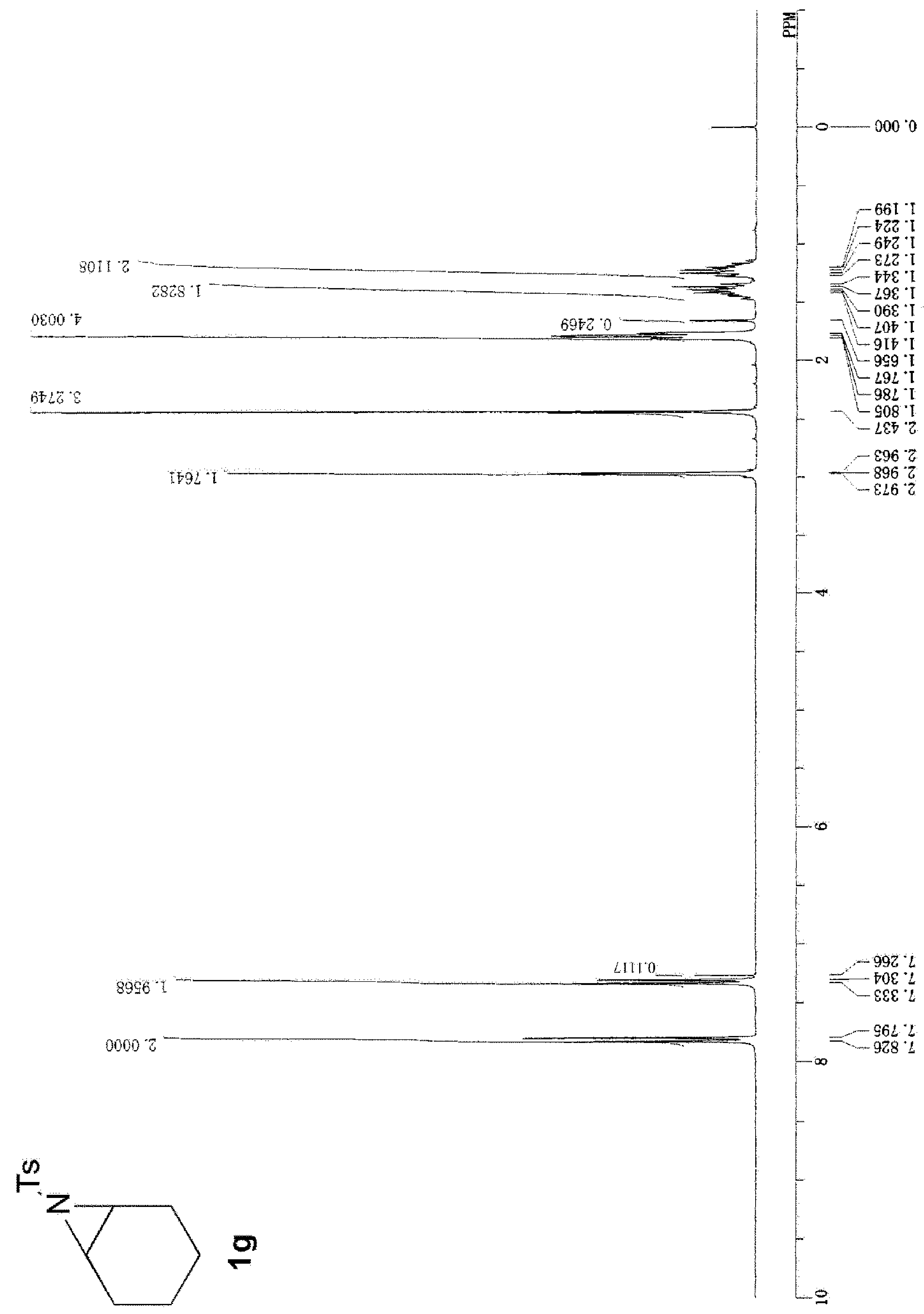




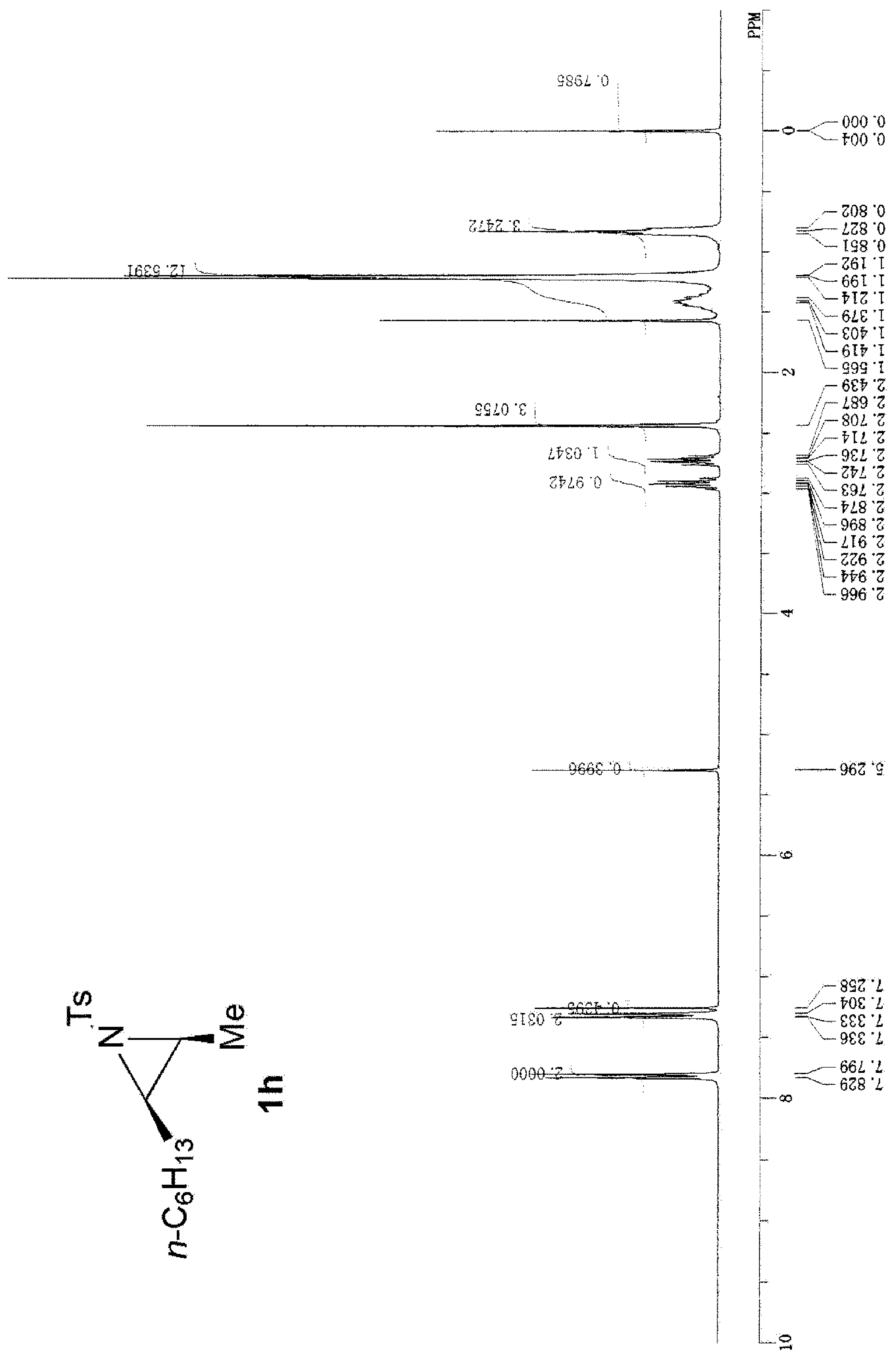




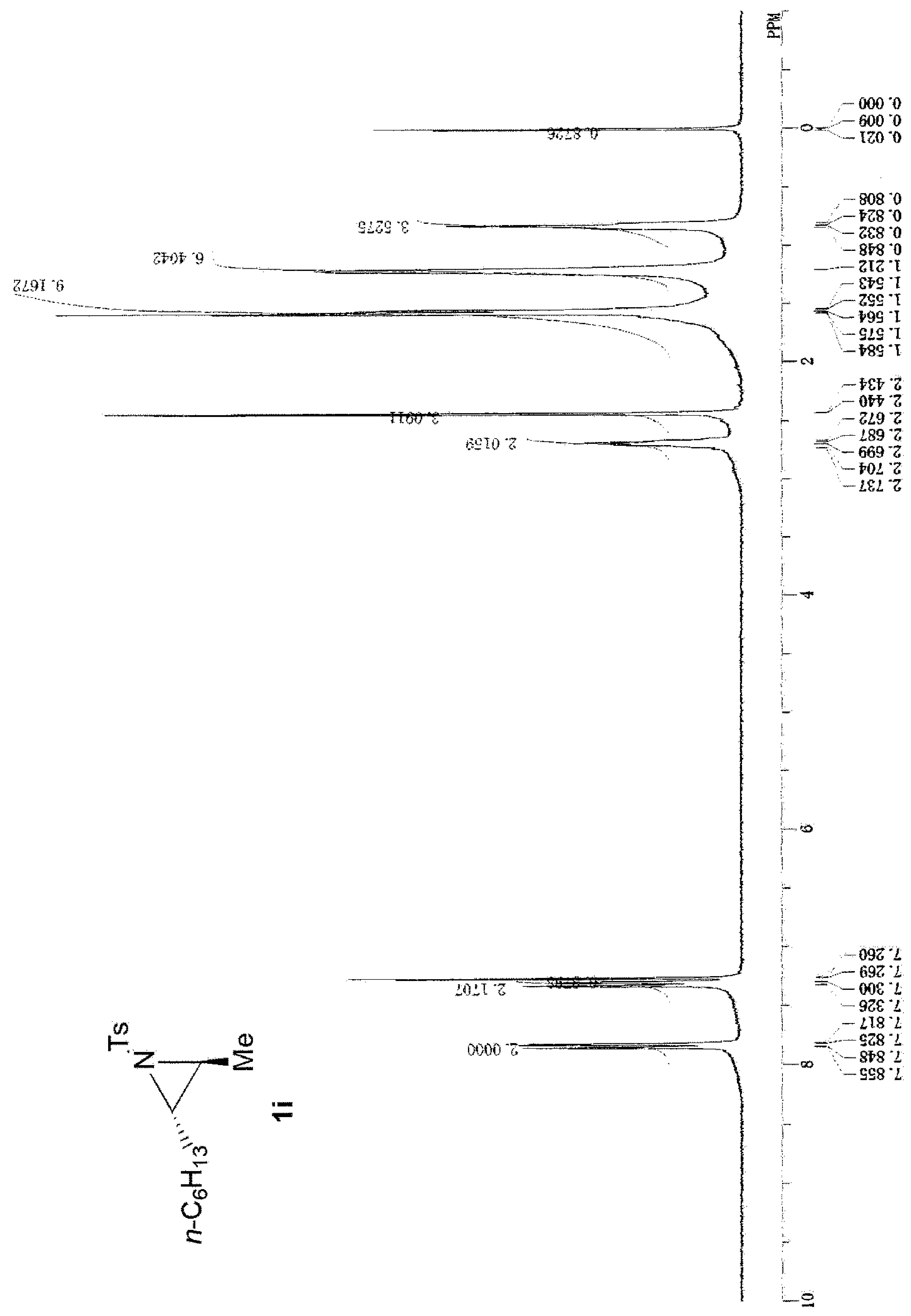

S21 


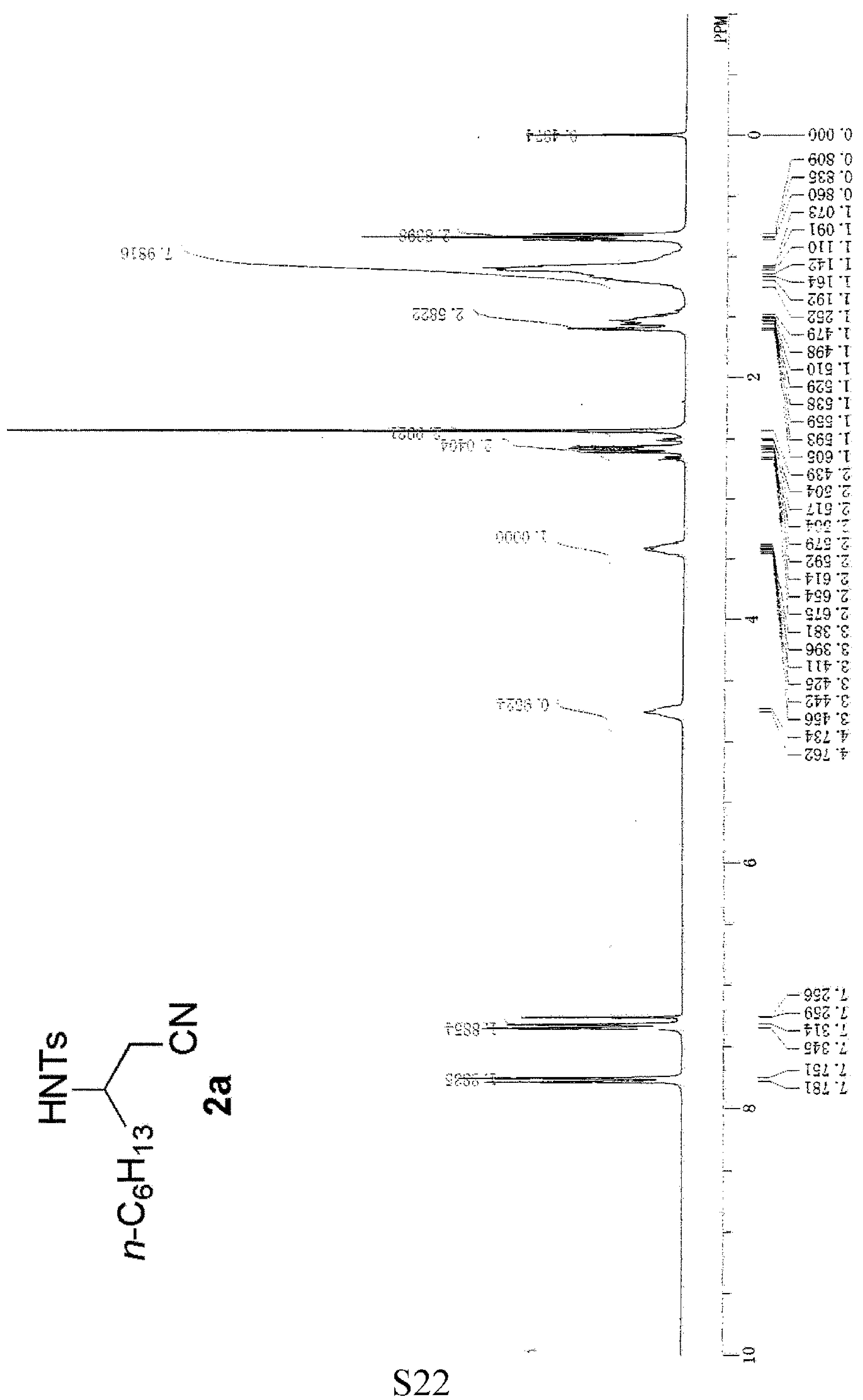




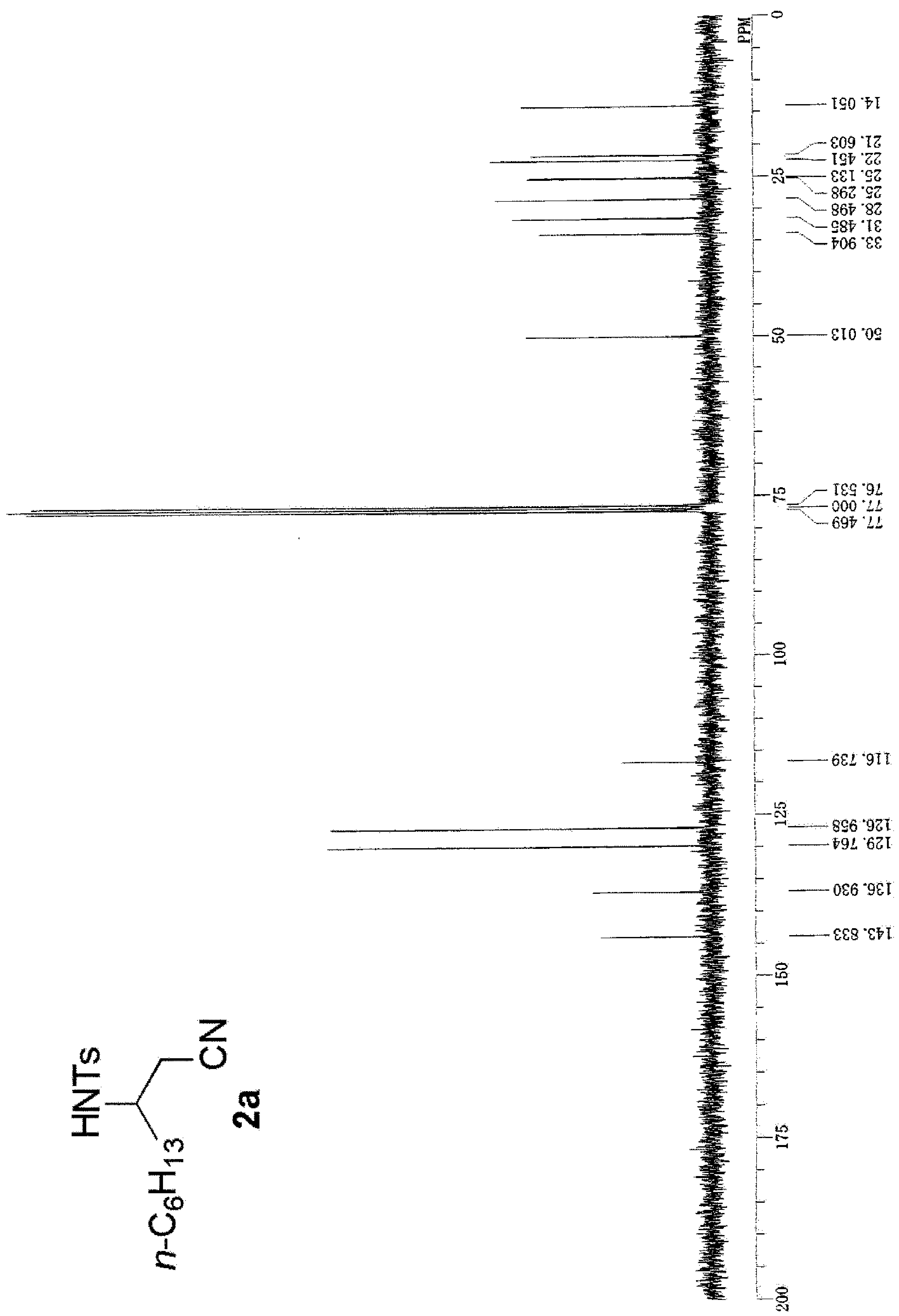




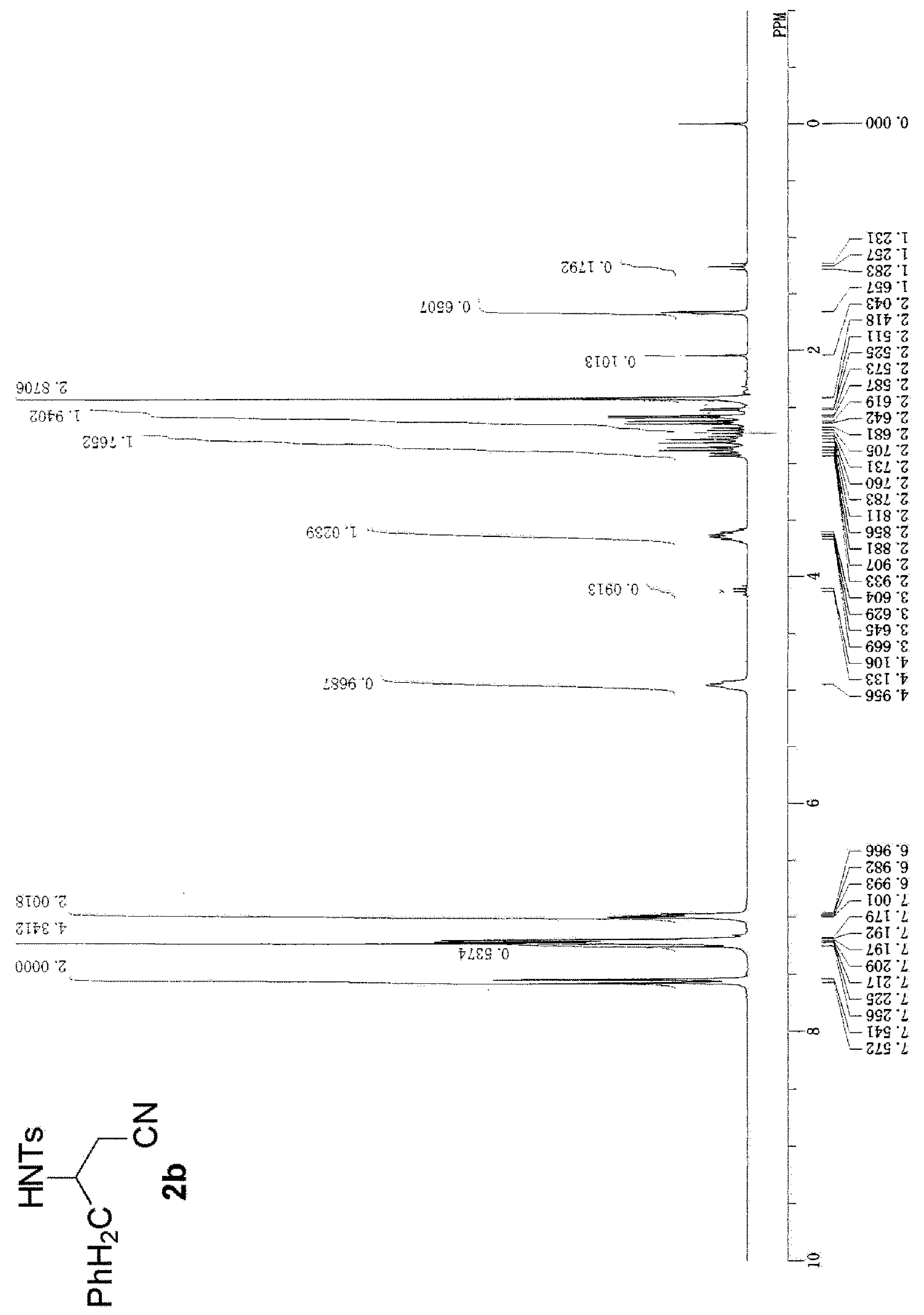




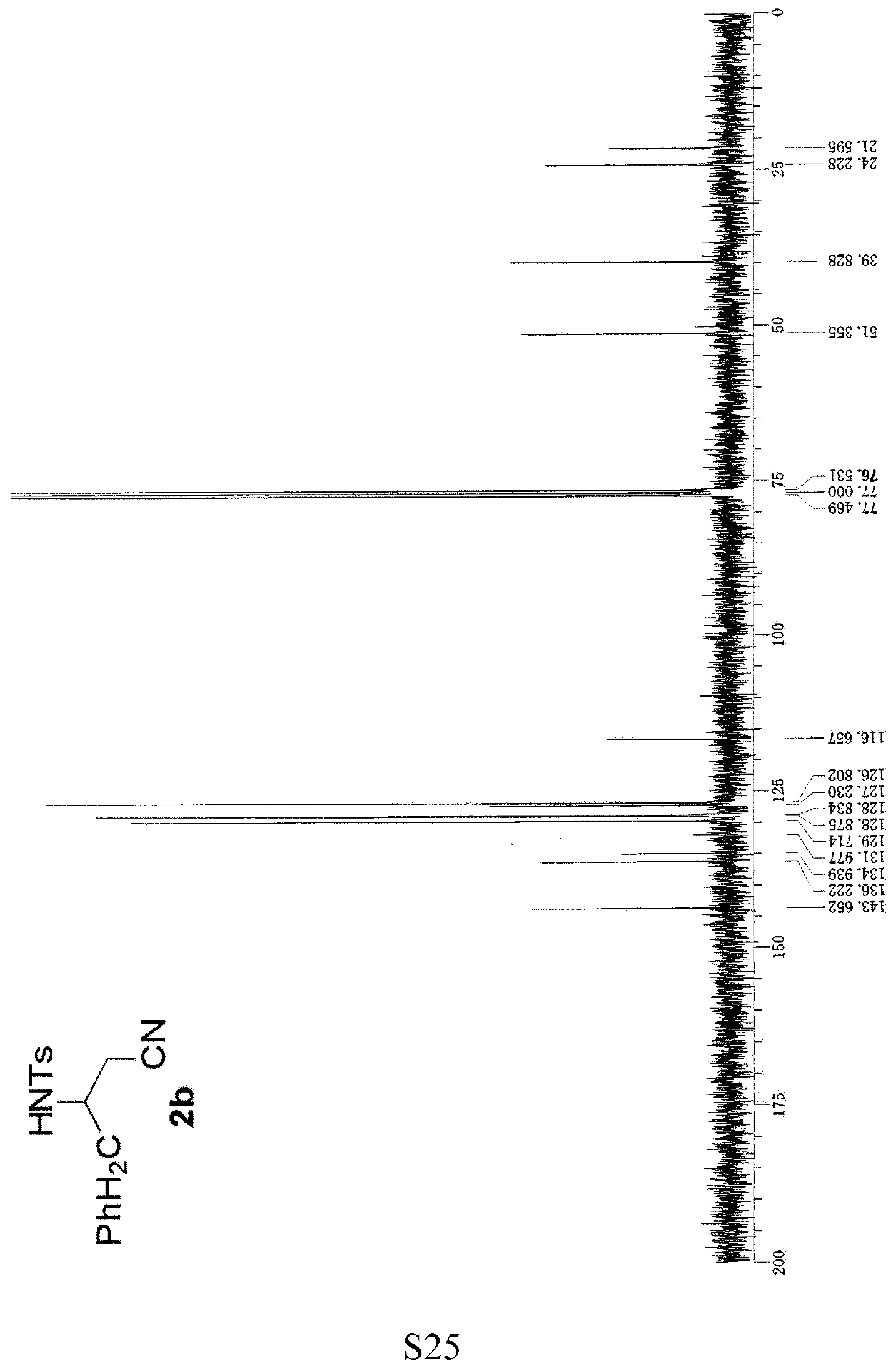




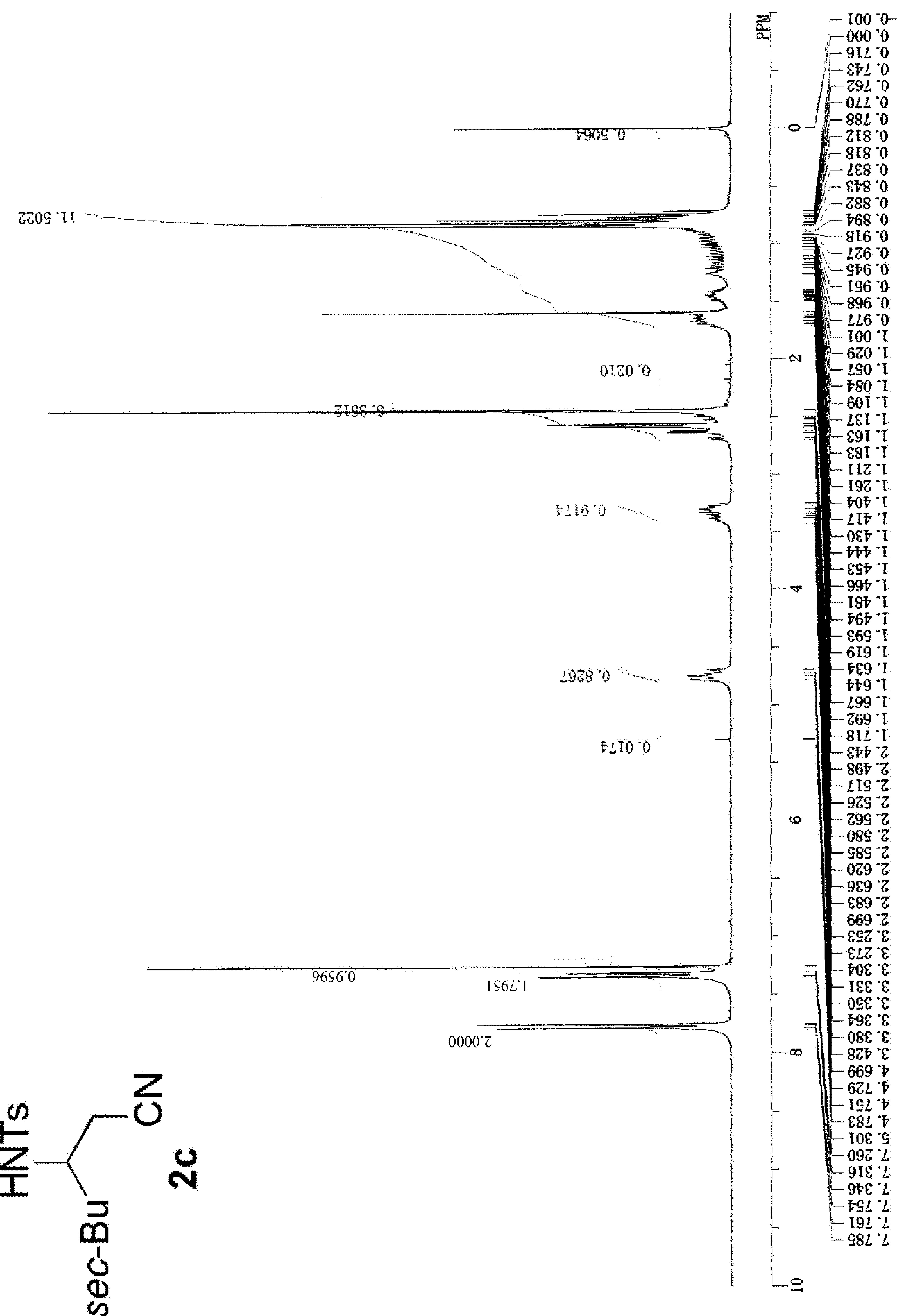




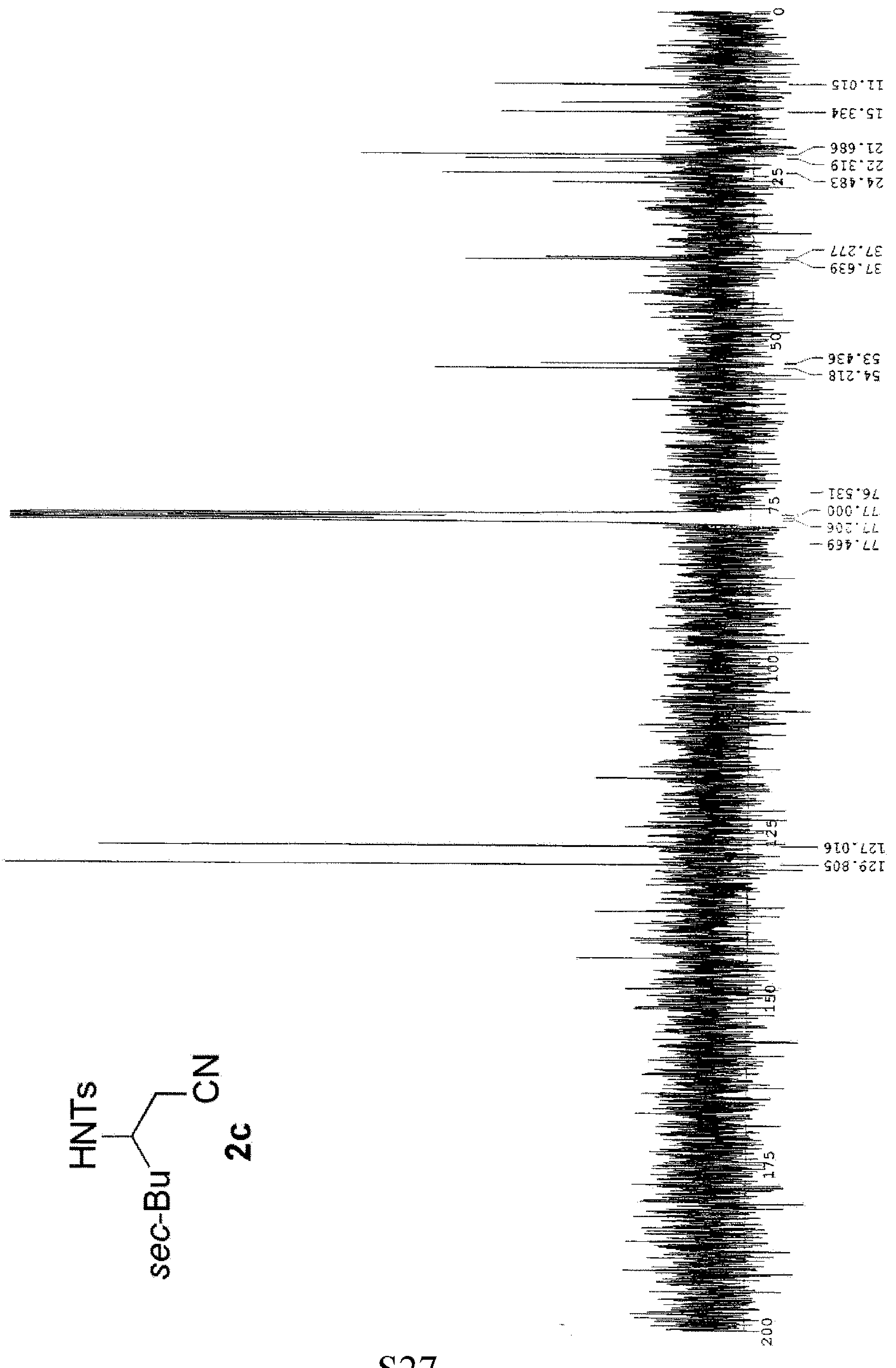




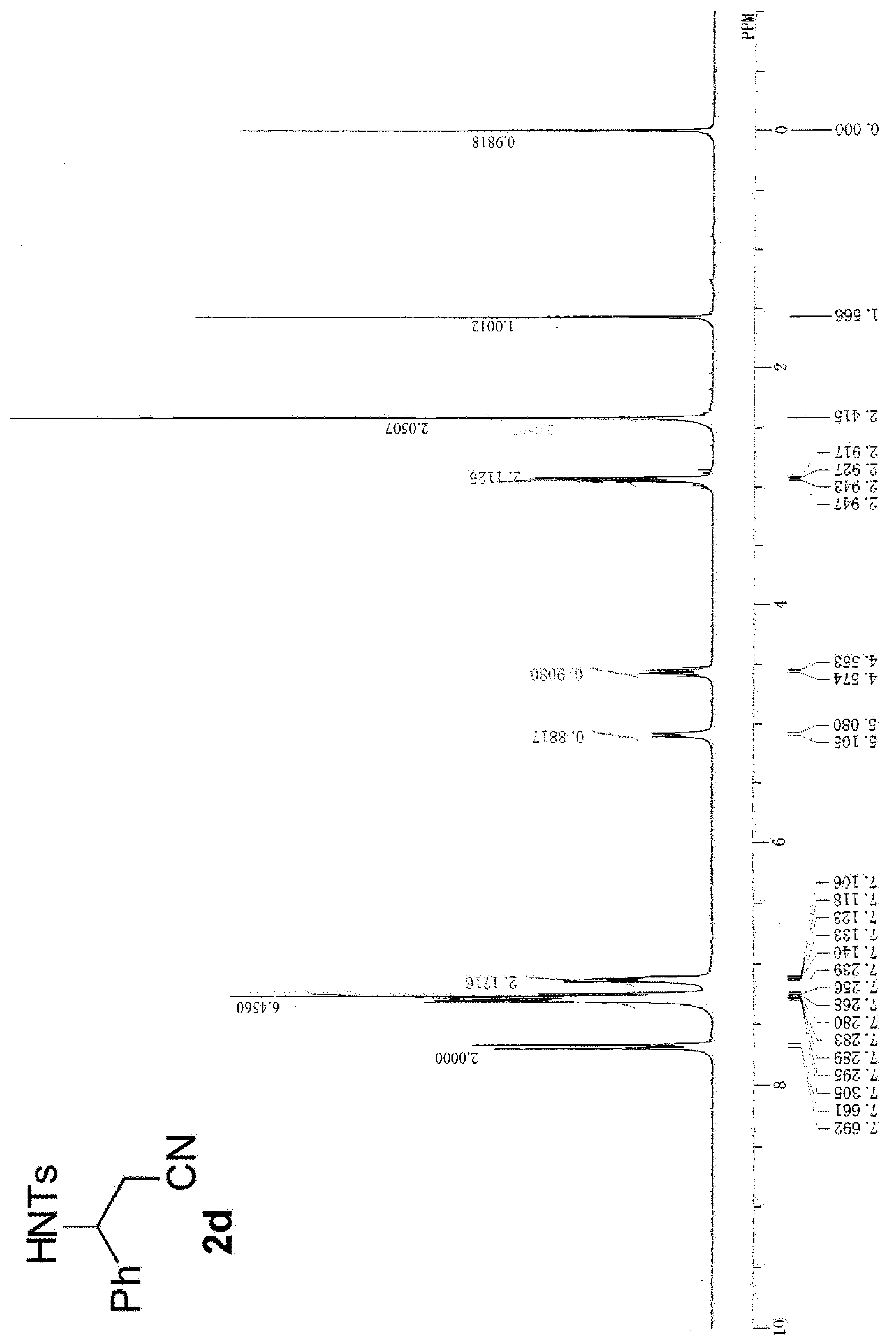

S28 


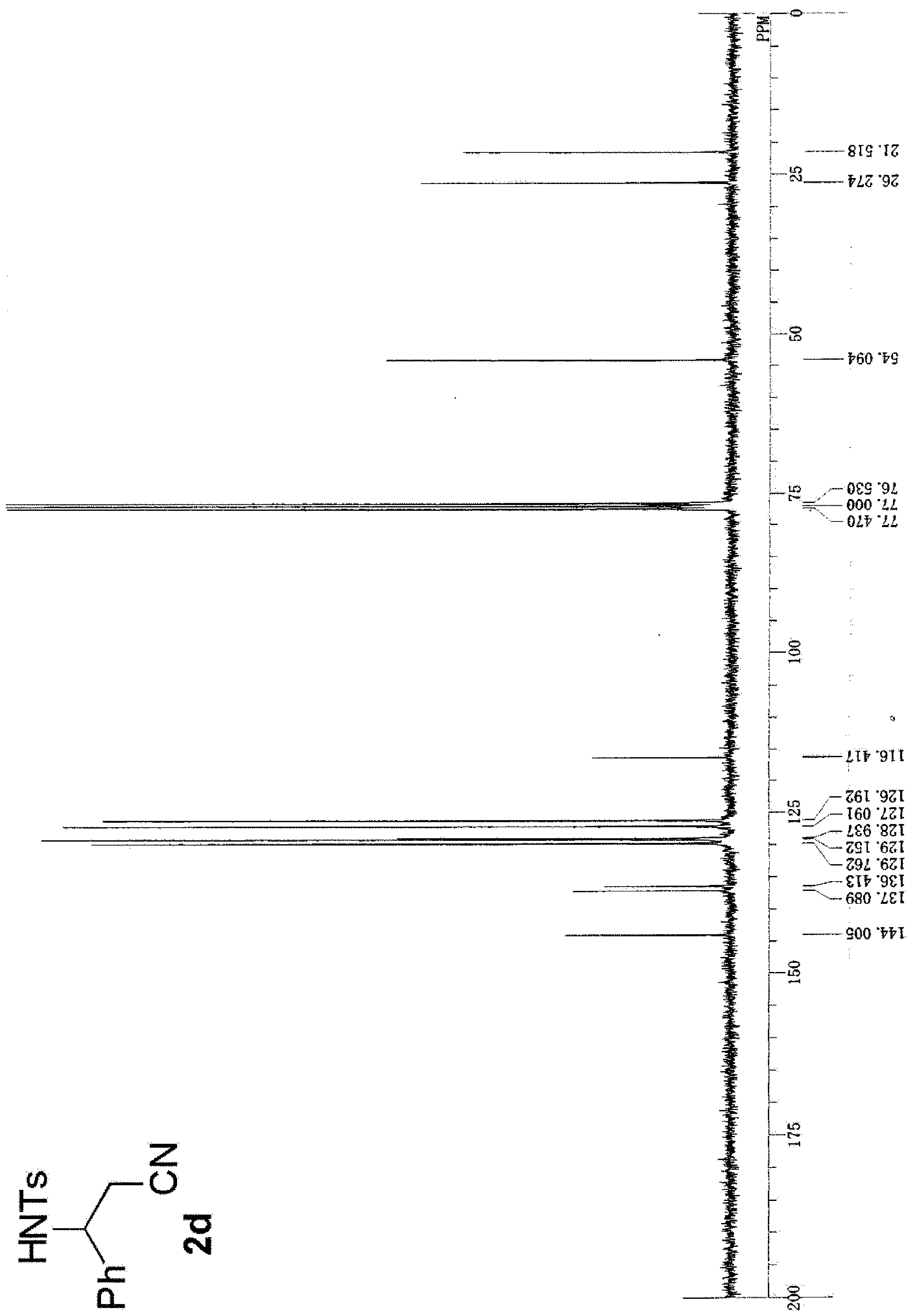

S29 


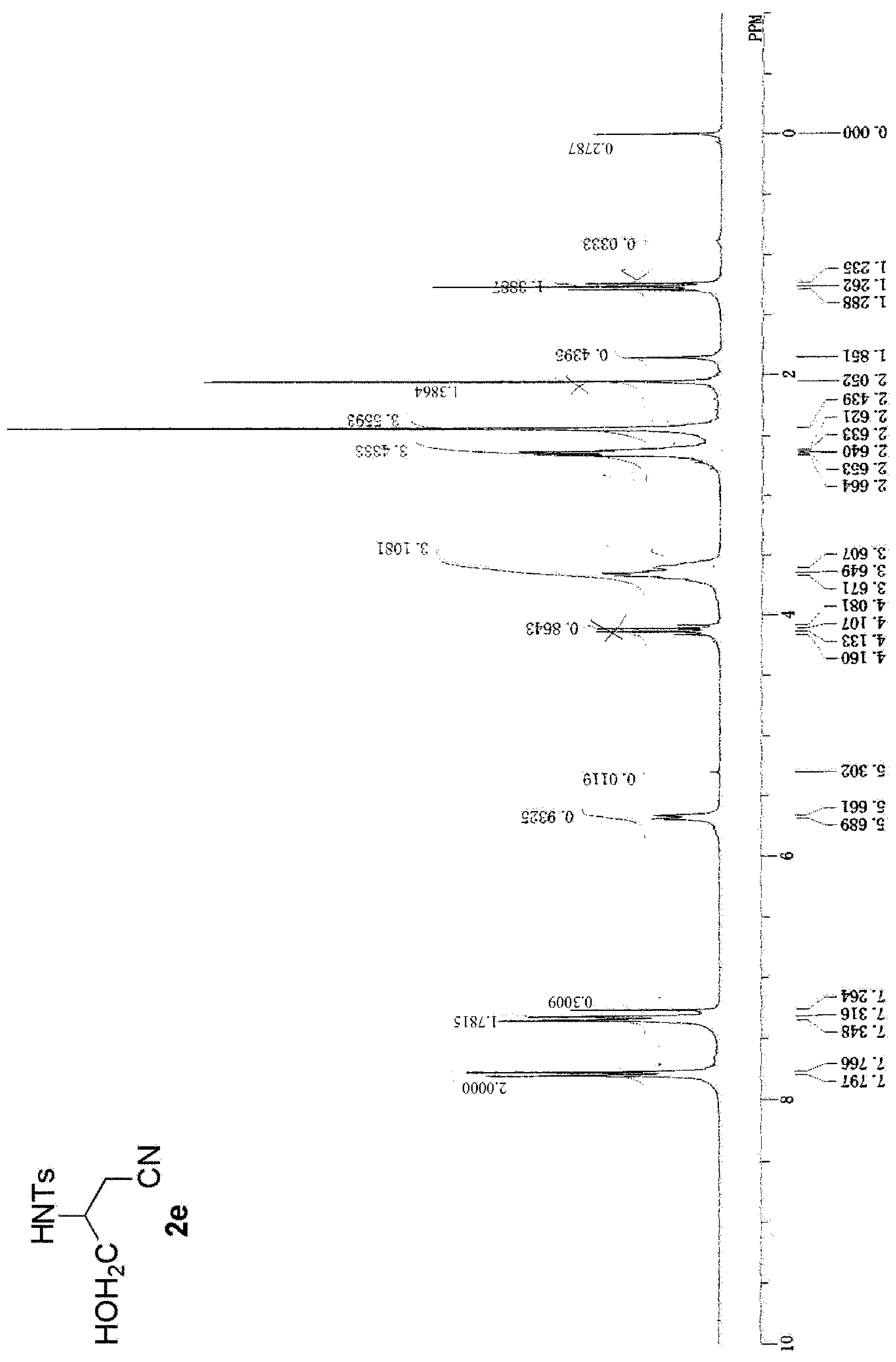




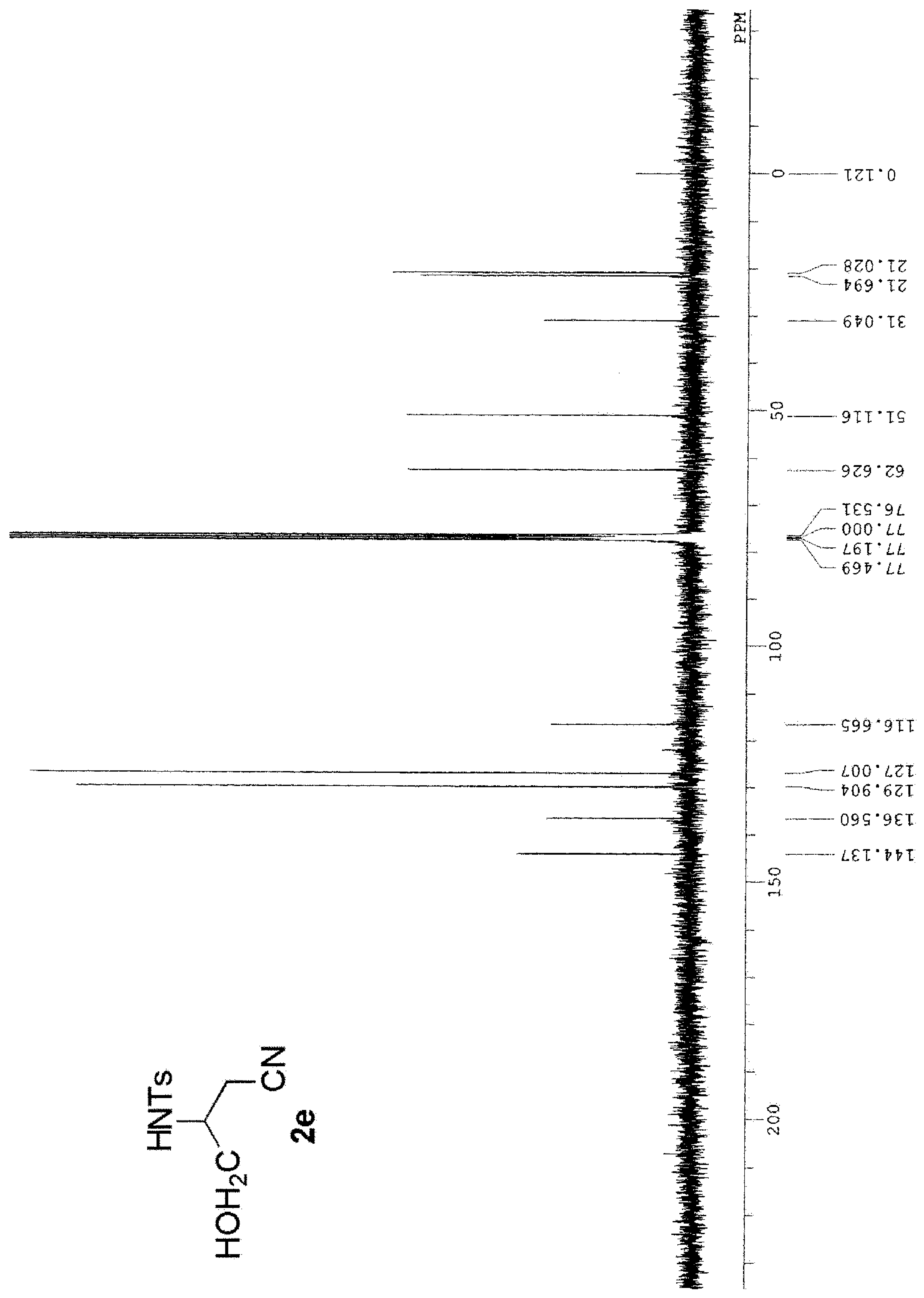

S31 


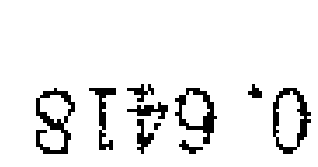

2000

$8619^{\circ}$

64190

99780

HTT

$0000^{\circ}$

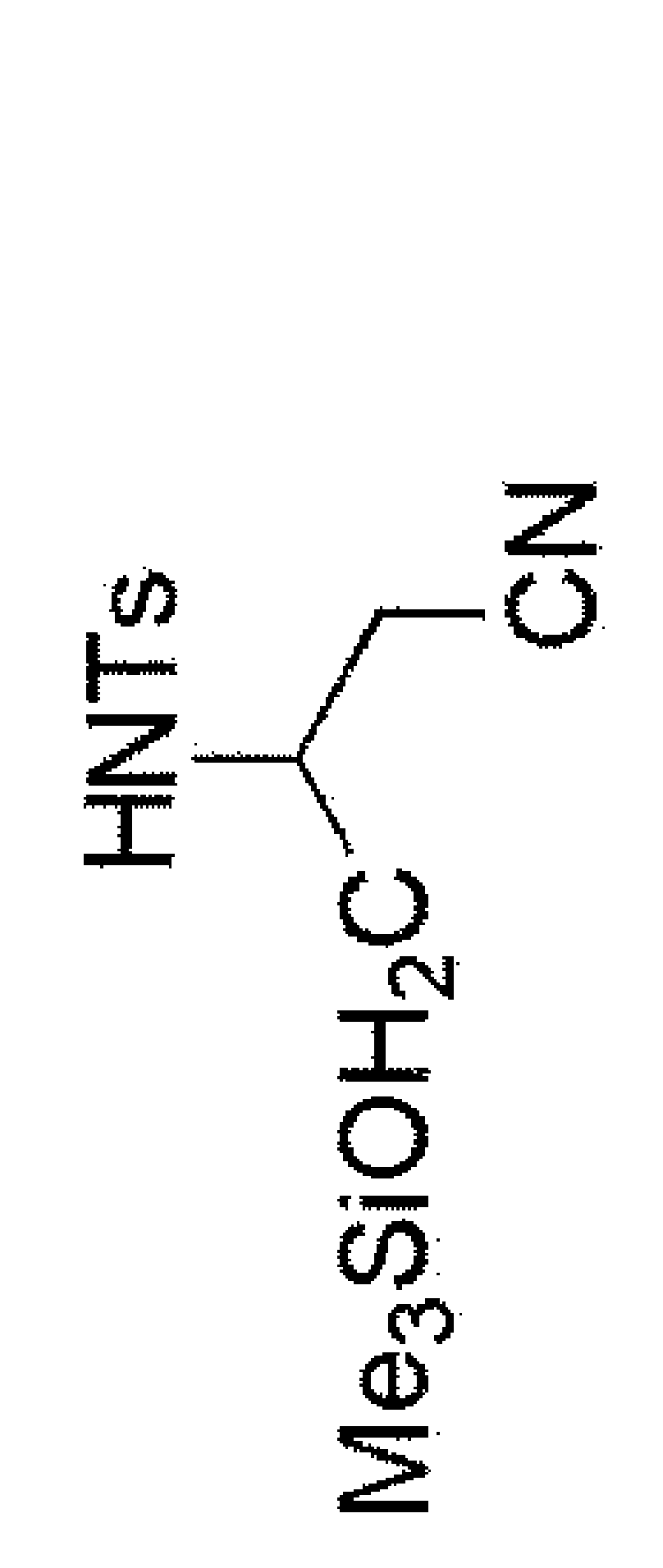

\section{0}

$-820^{\circ} 0-$

$-390^{\circ} 0$

$8200^{\circ}$

$000^{\circ} 0$

$210^{\circ} 0$

$-870^{\circ} 0$

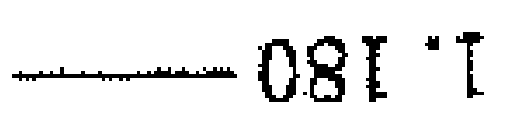

$-\infty$

$-1 L E \cdot$

$-2 \varepsilon 9^{\circ} Z$

$-609$

$-79 \cdot z$
$-699^{\circ}$

$-16 \varepsilon^{\circ} \varepsilon$
$-96 \varepsilon^{\circ} \varepsilon$

- 015

- -129.

$-1 e^{\circ}$

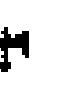

$-299^{\circ} \varepsilon$

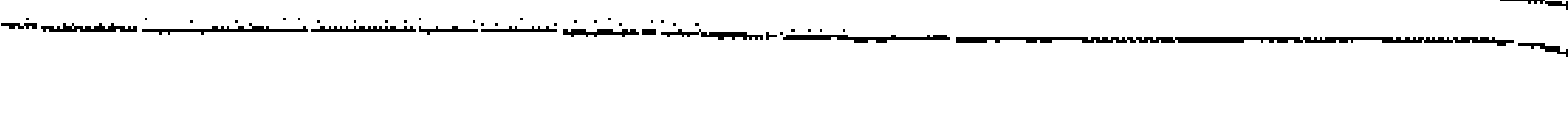




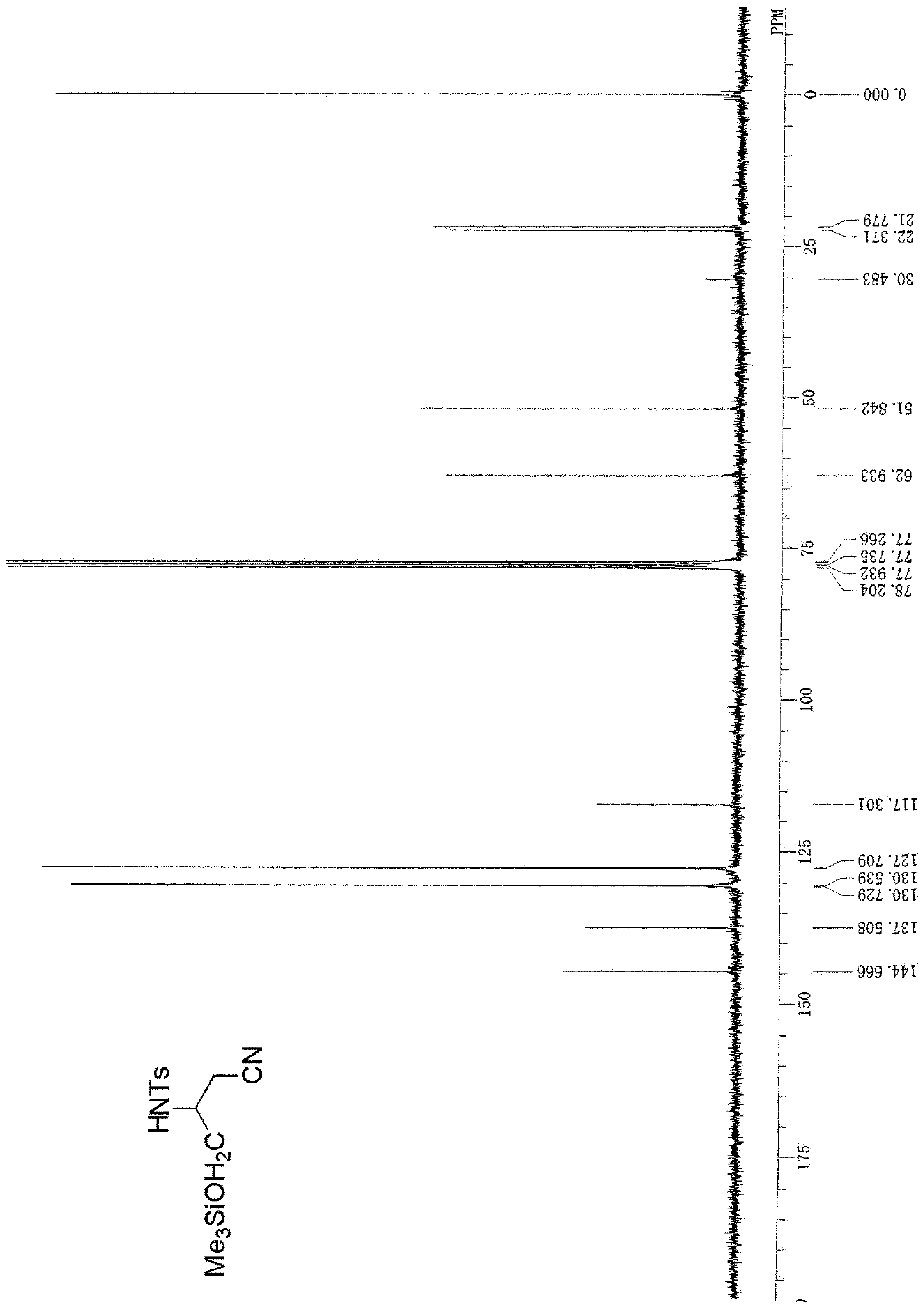

S33 


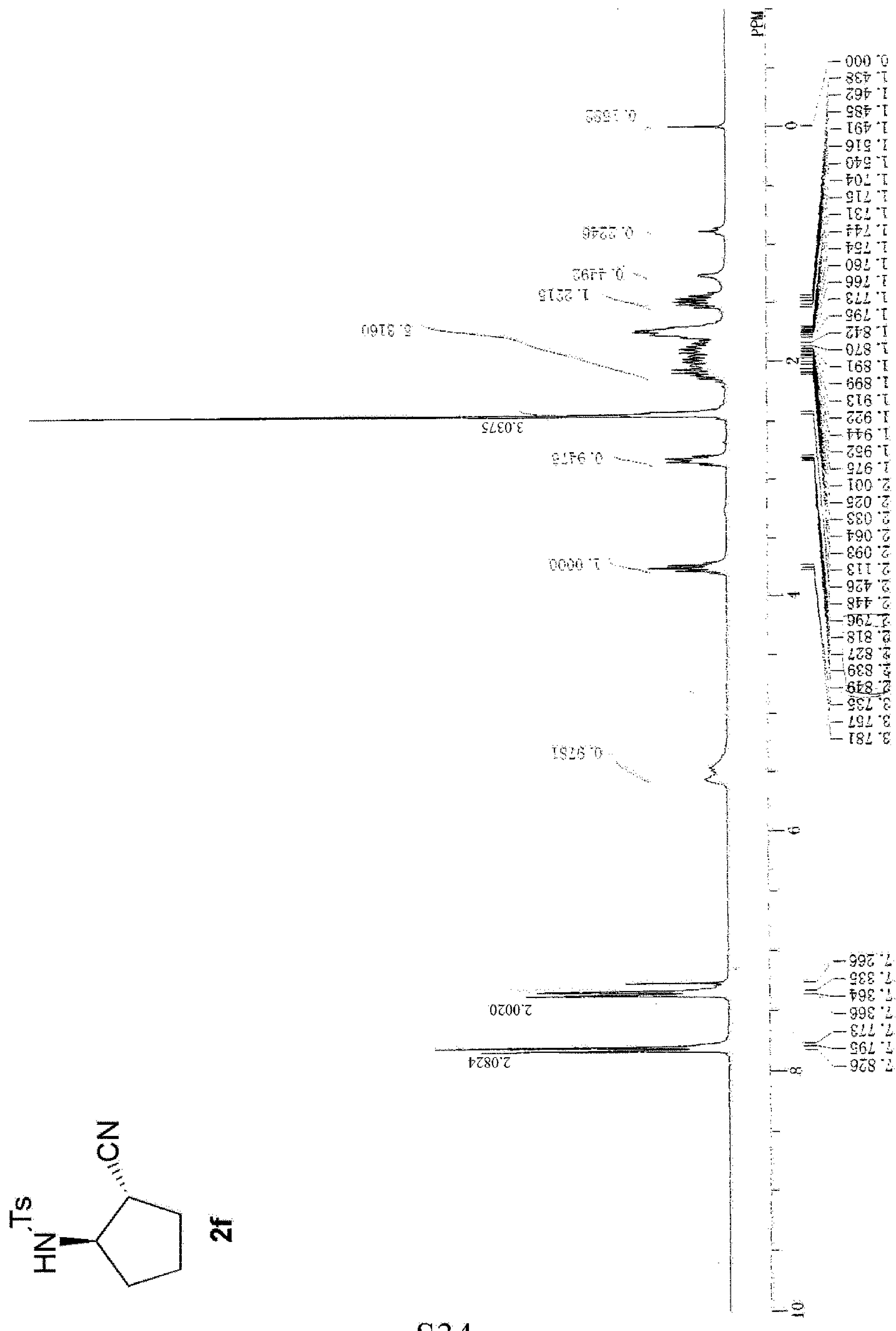




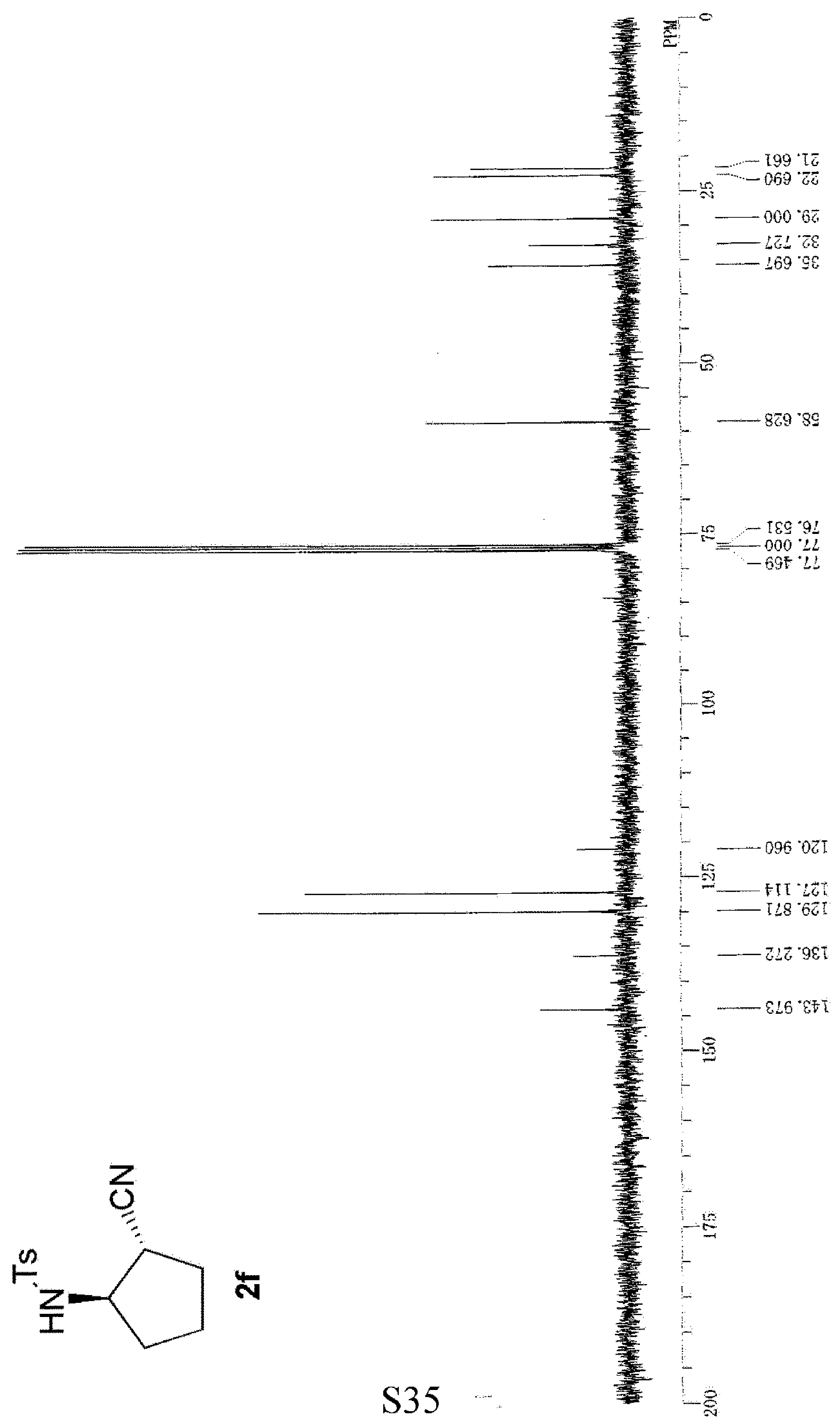




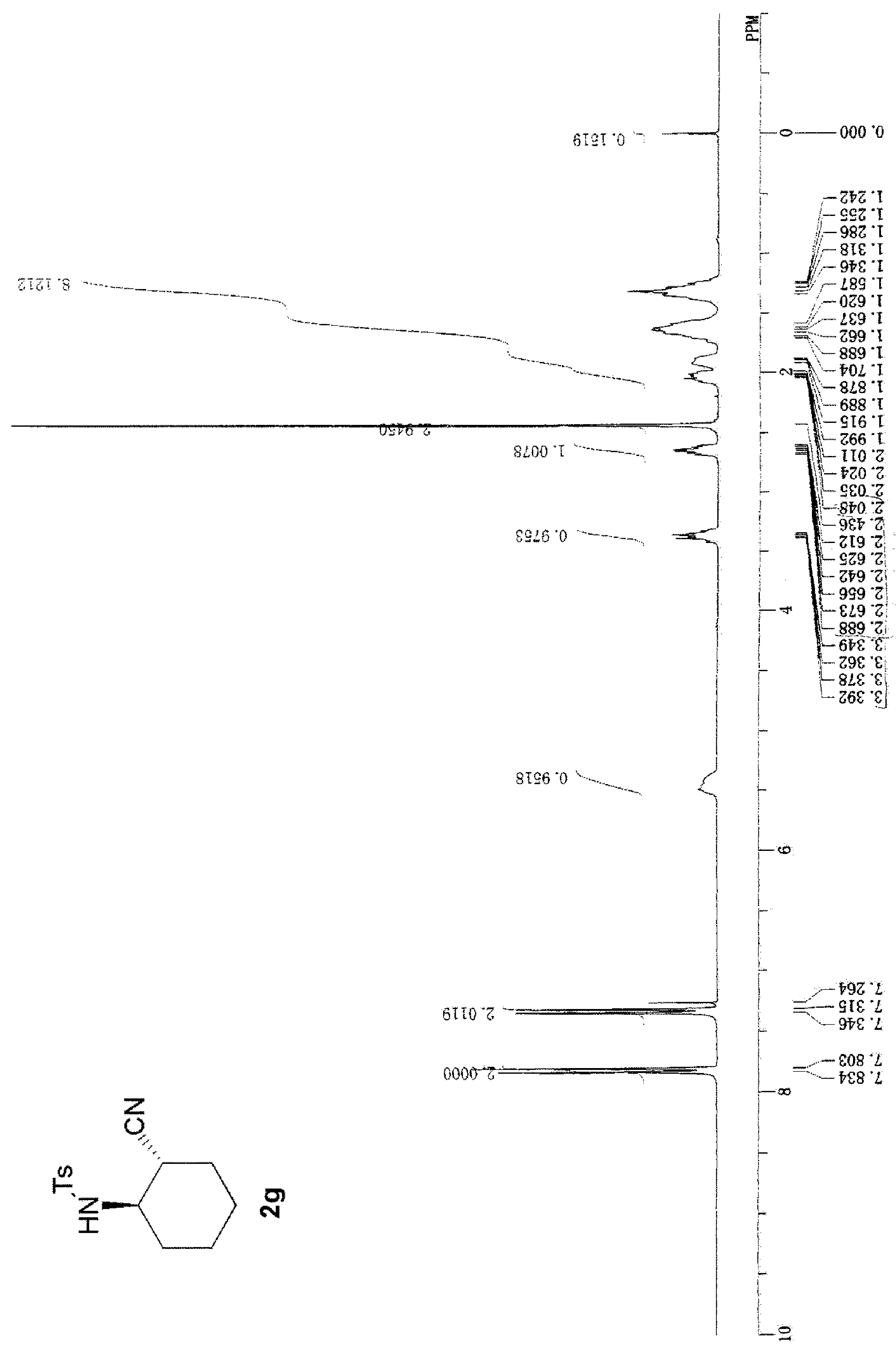




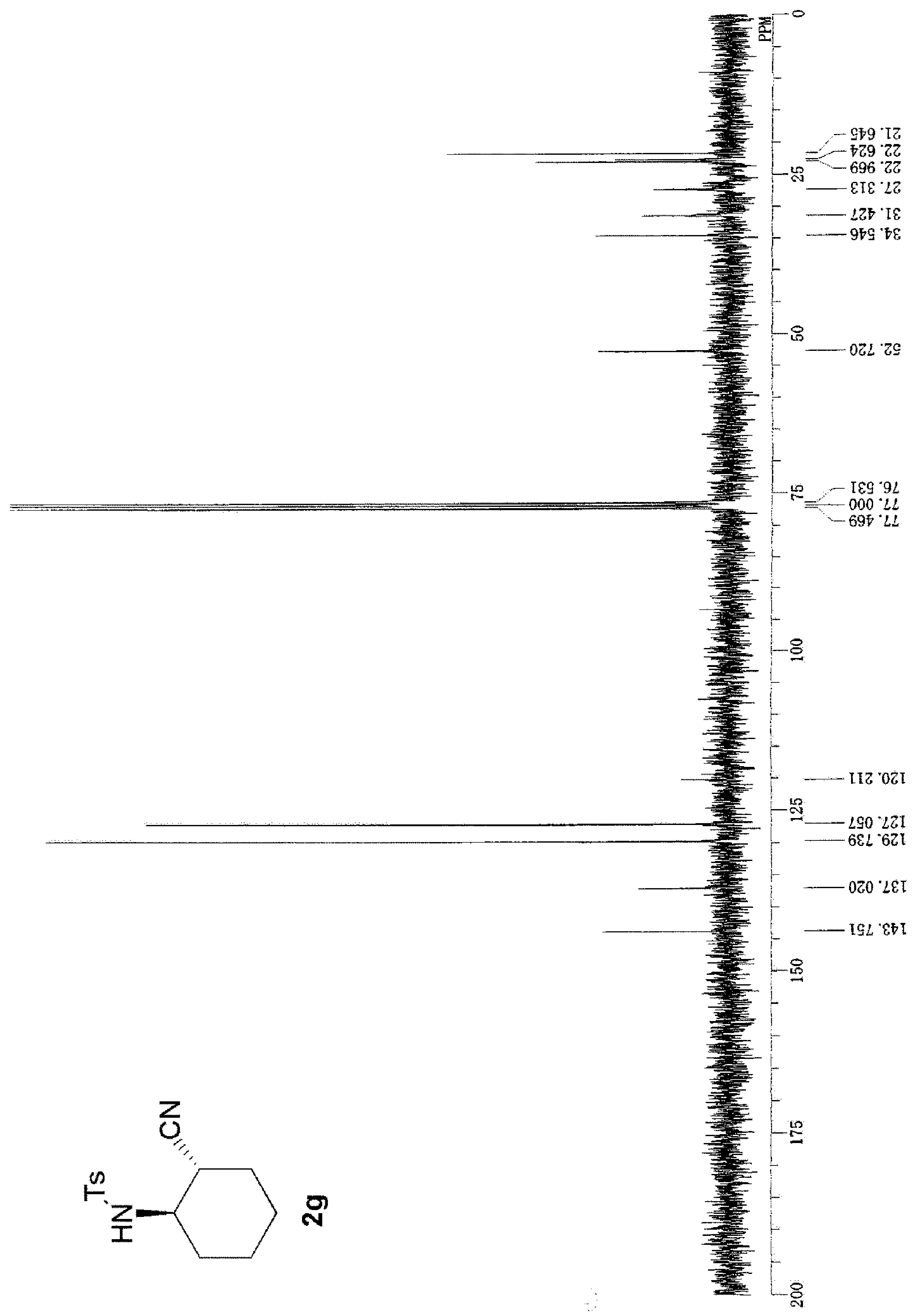



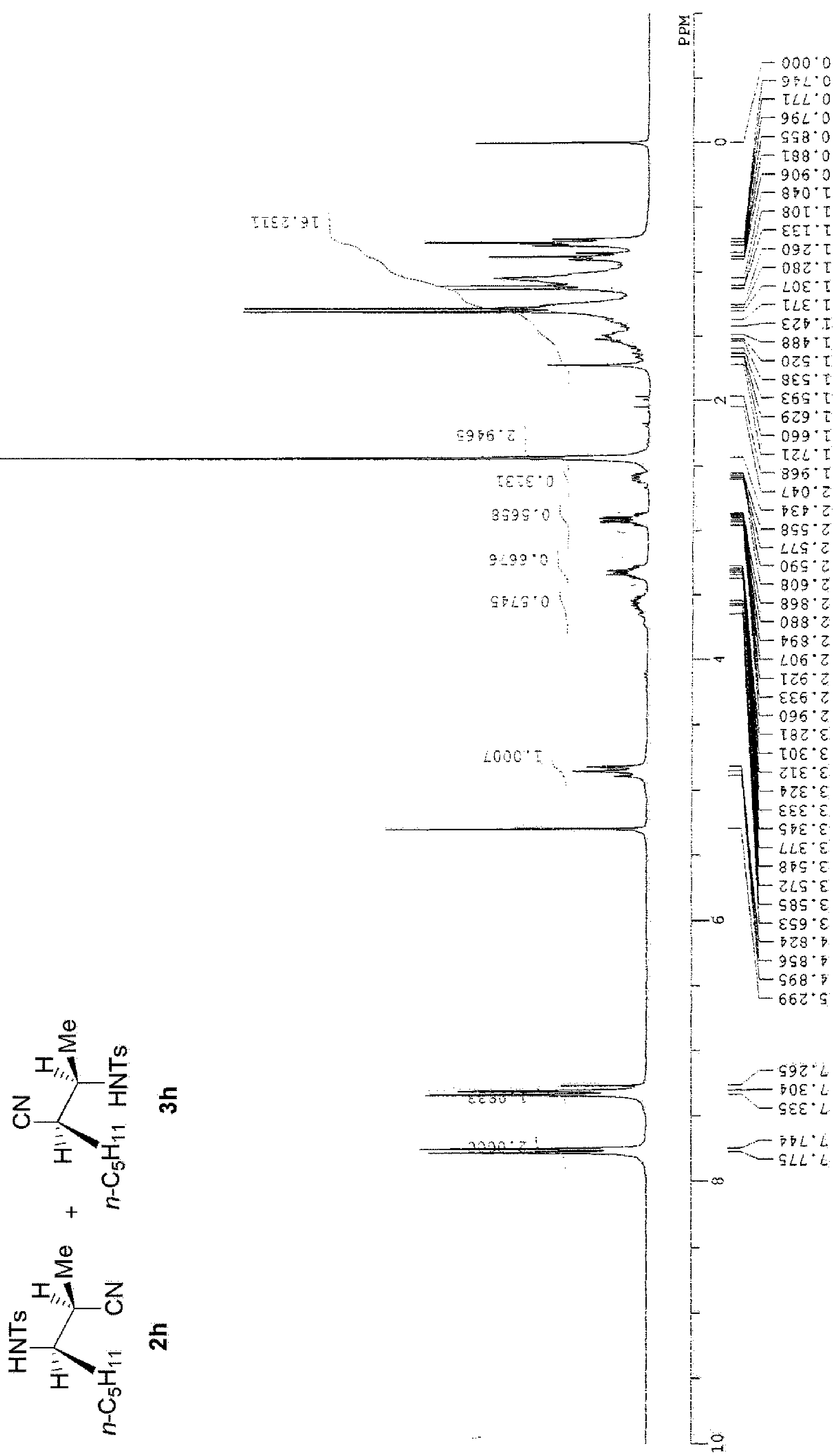


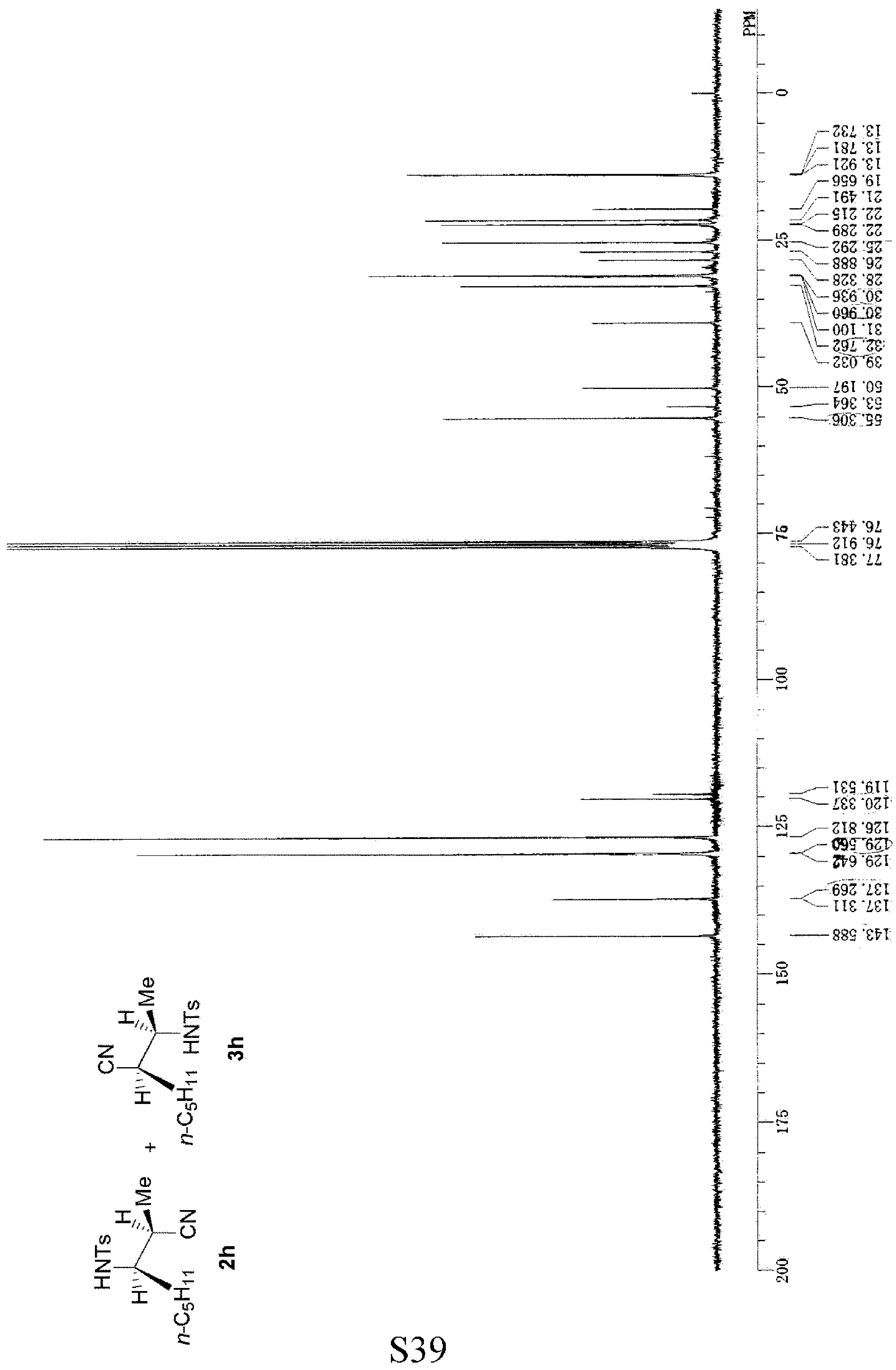




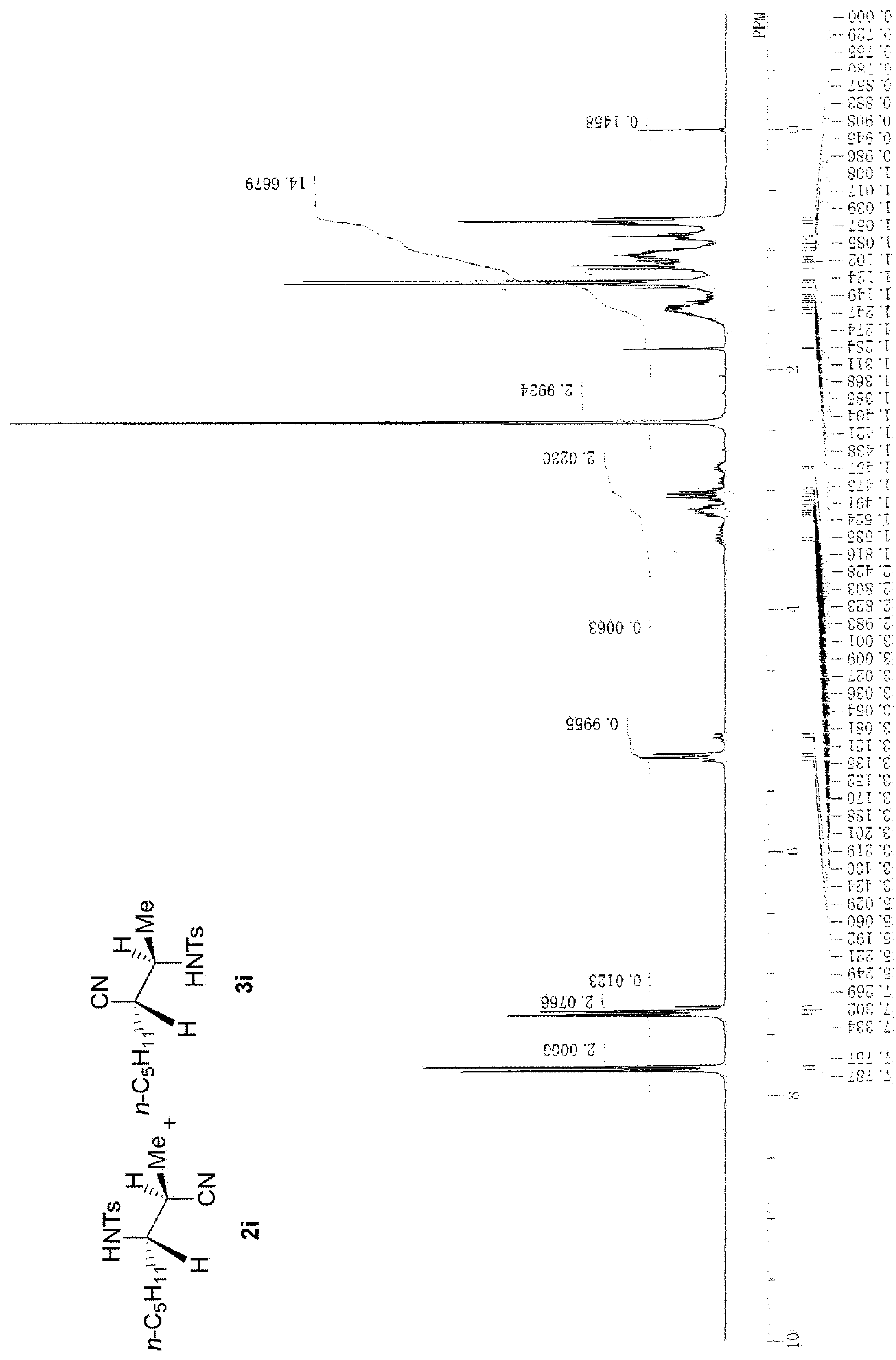




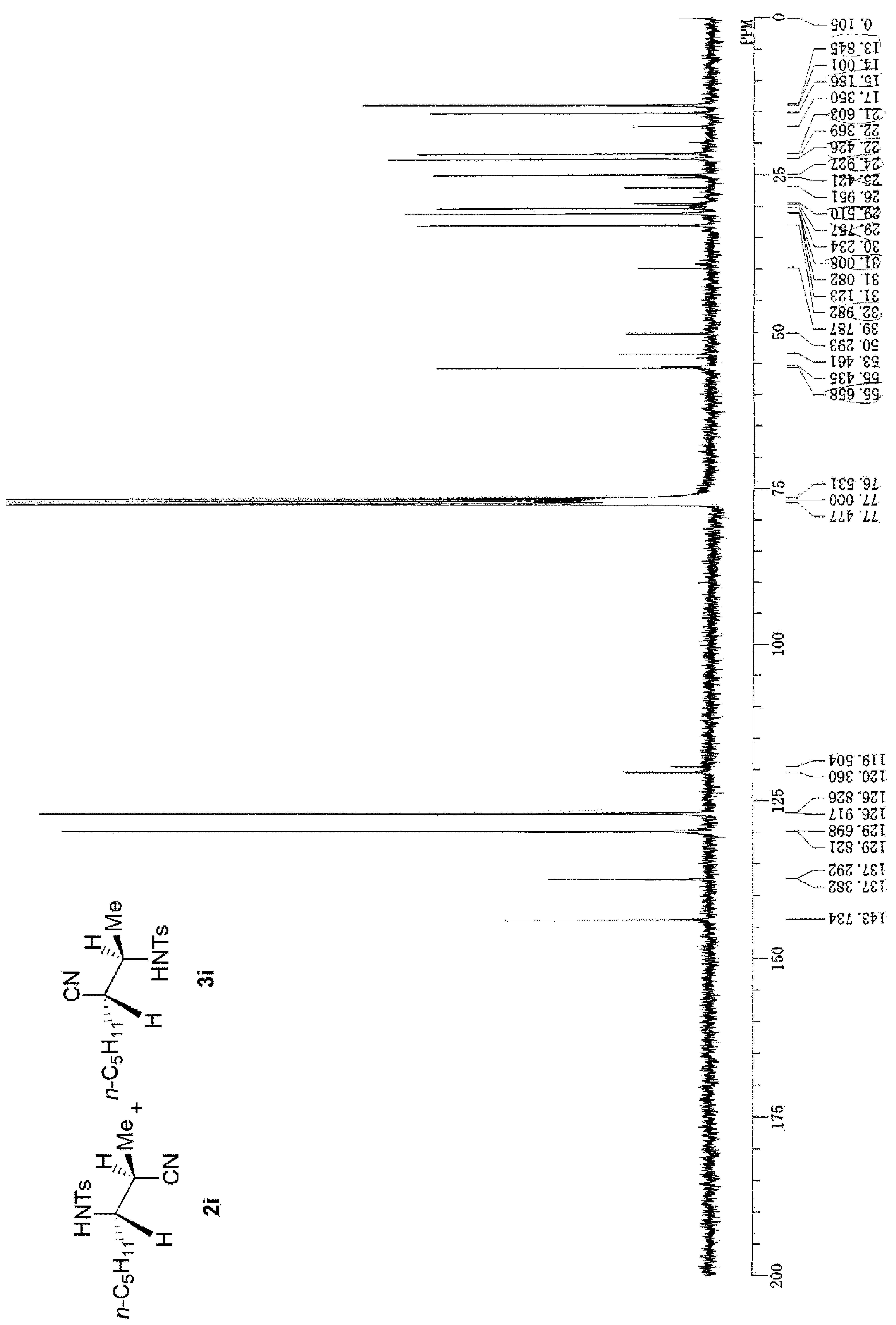




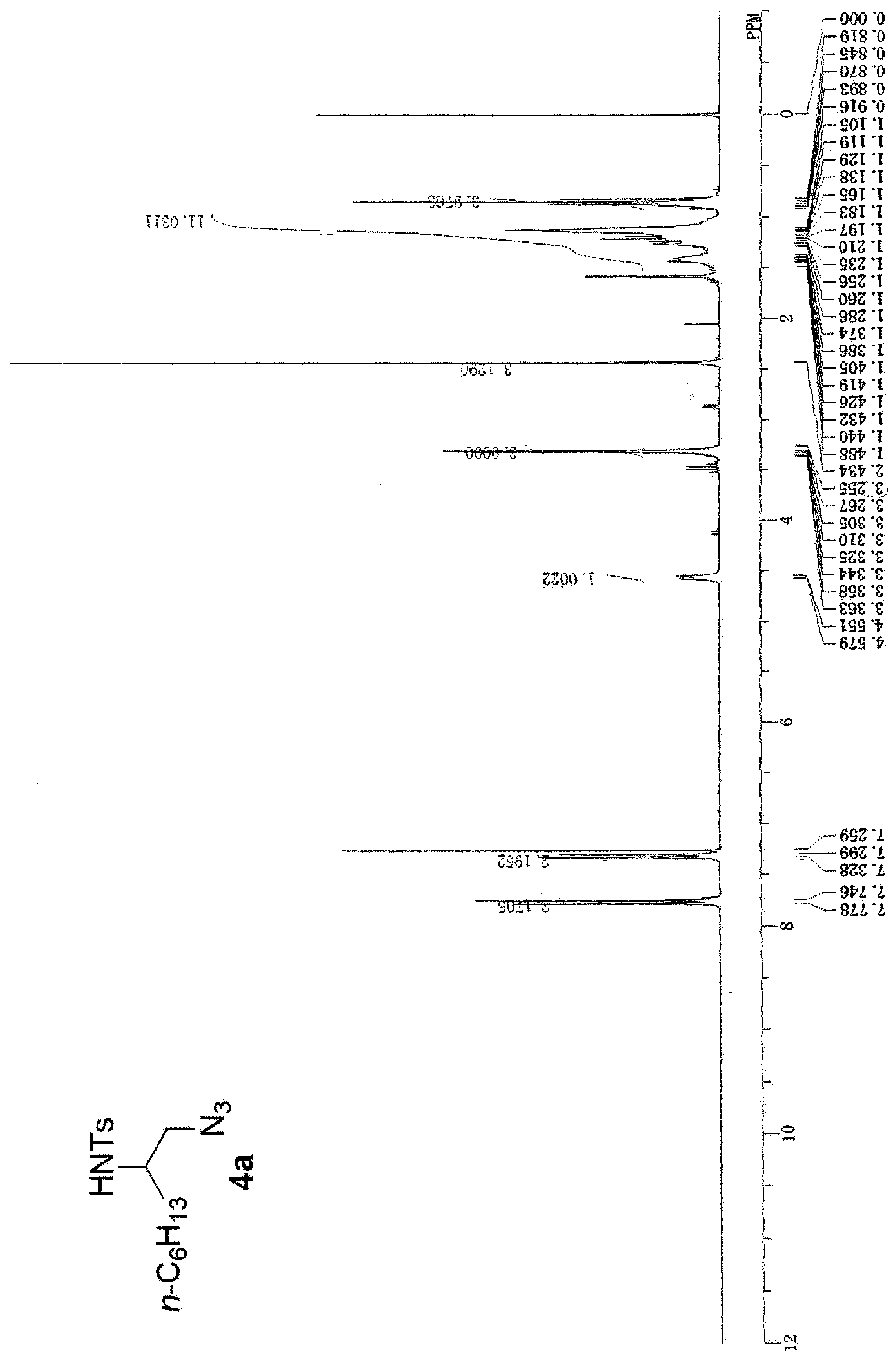

S42 


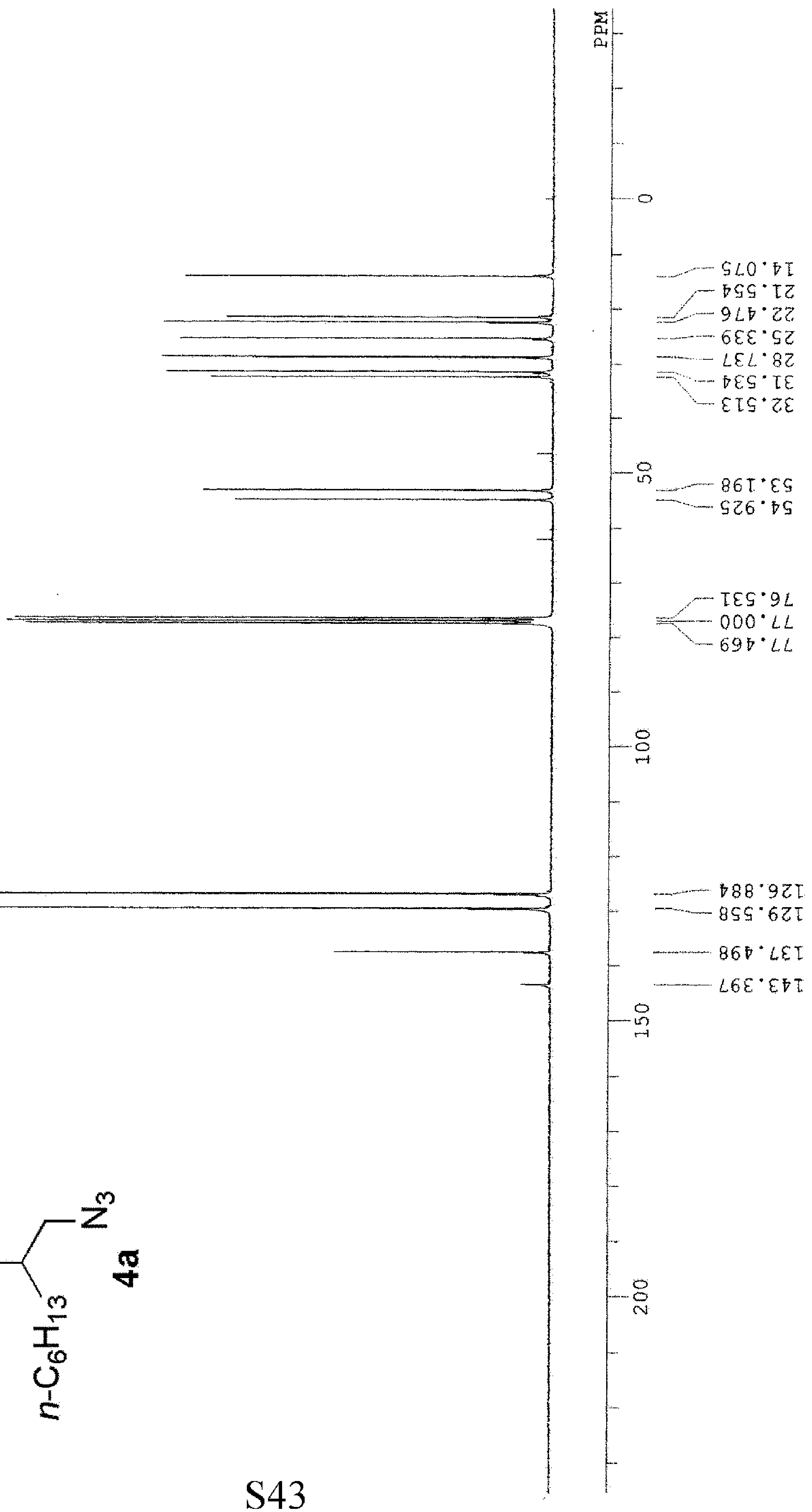




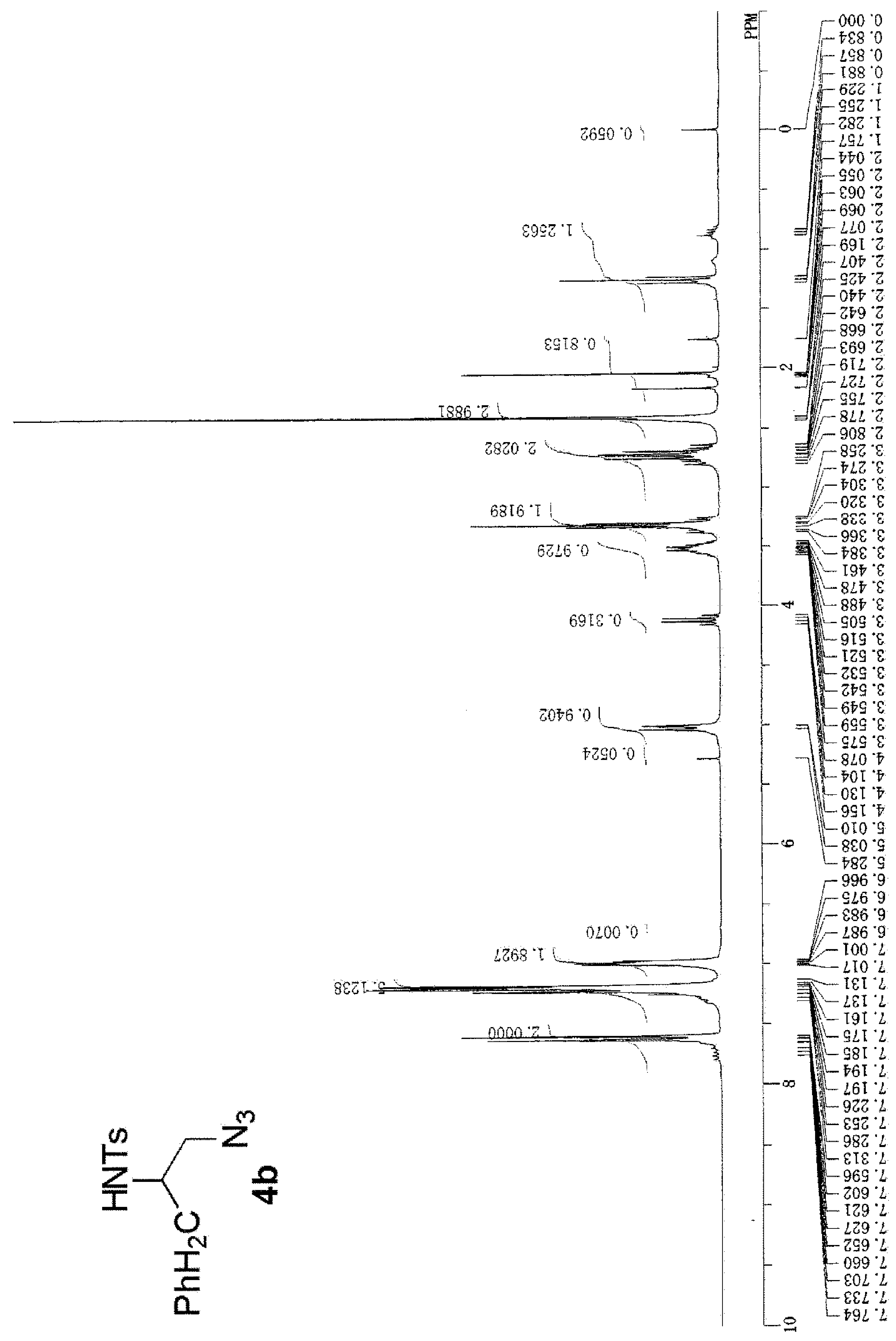




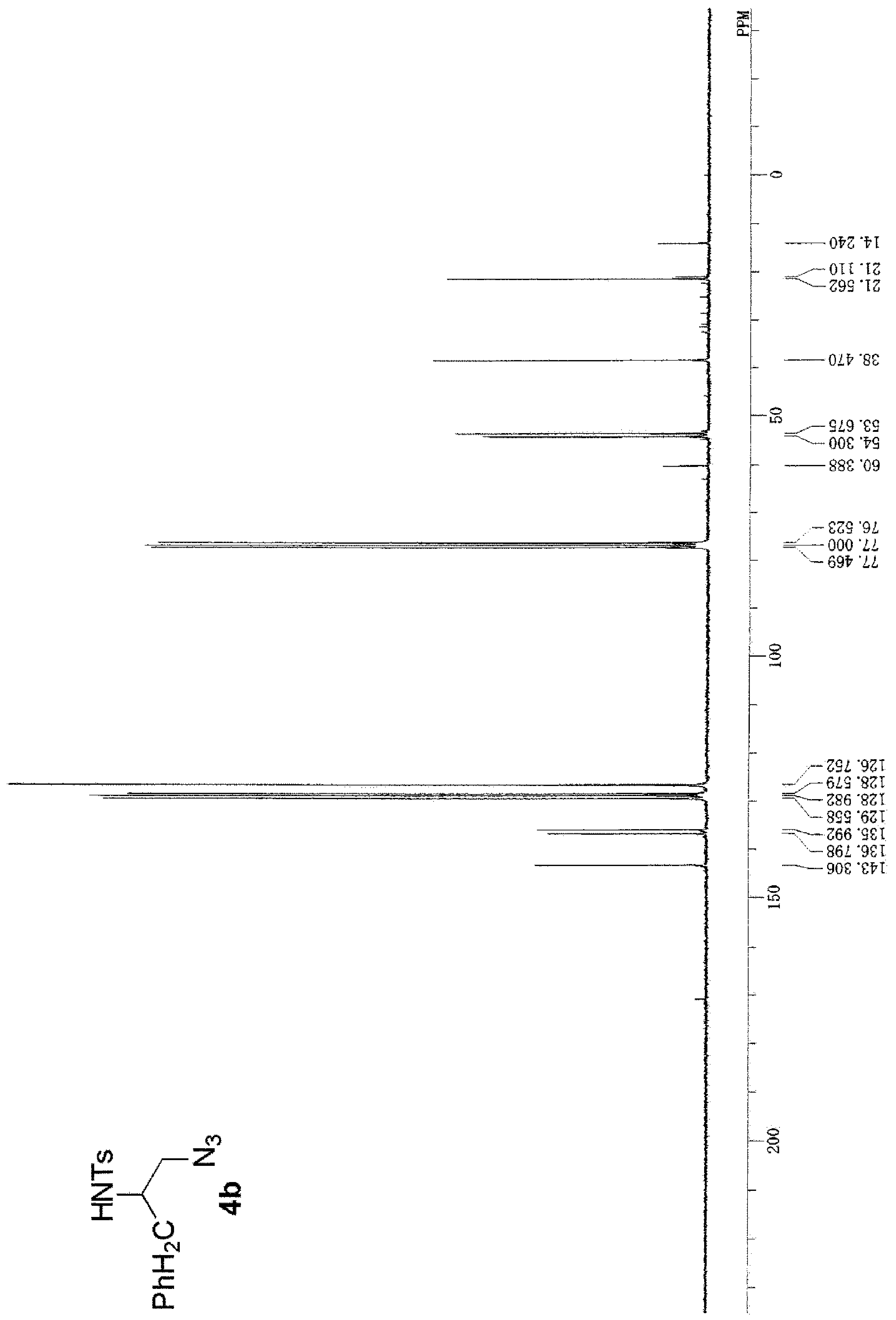




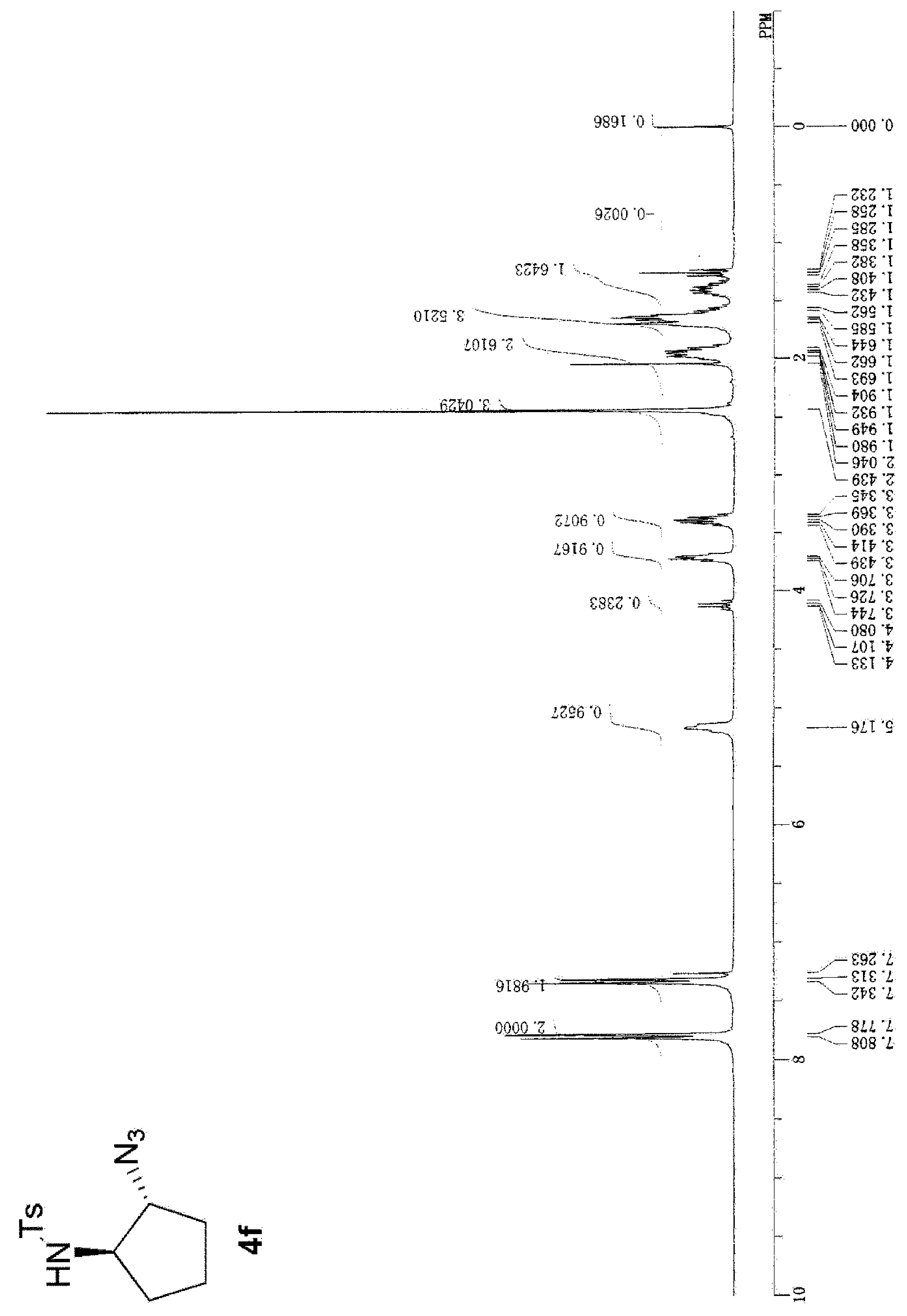




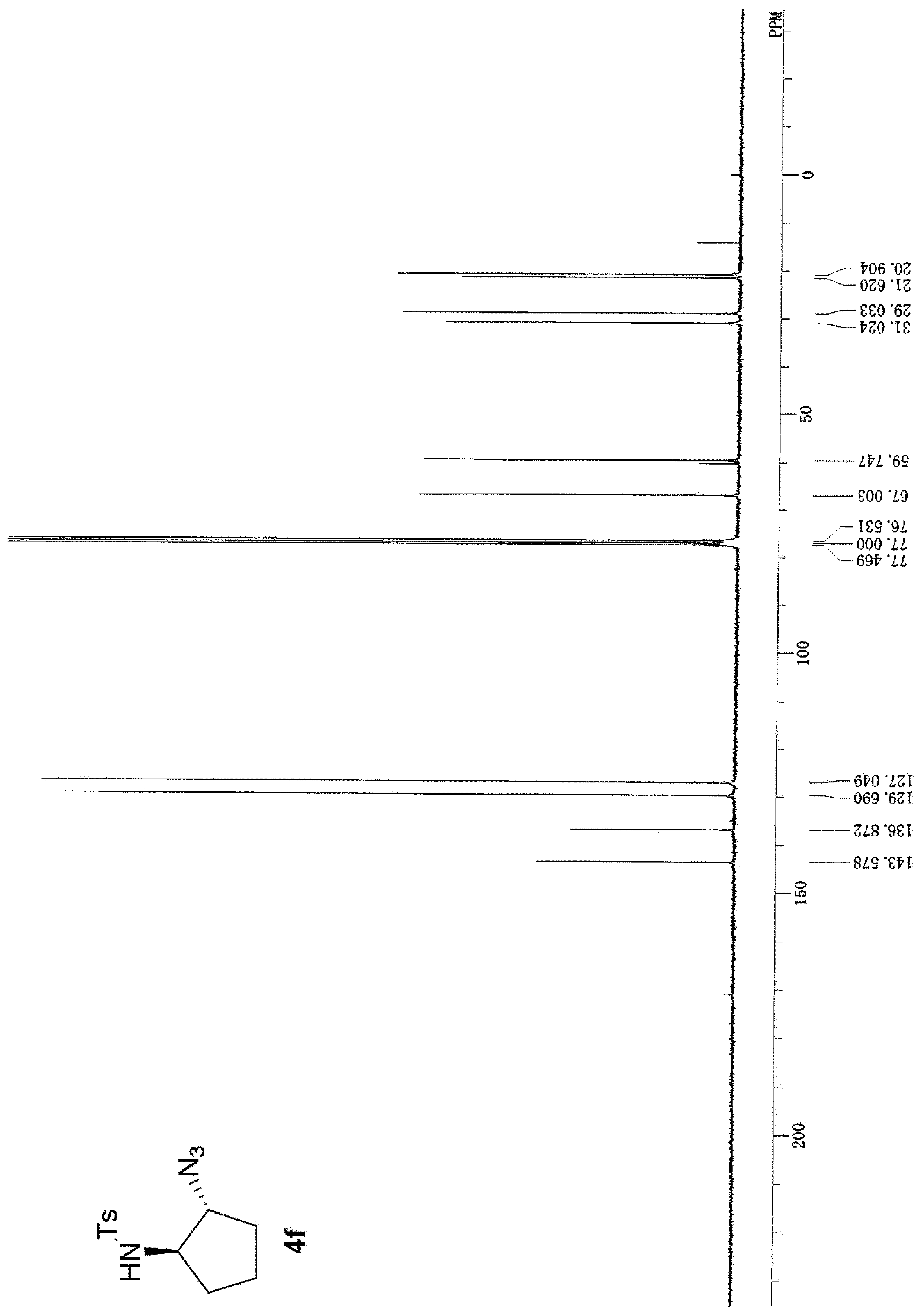




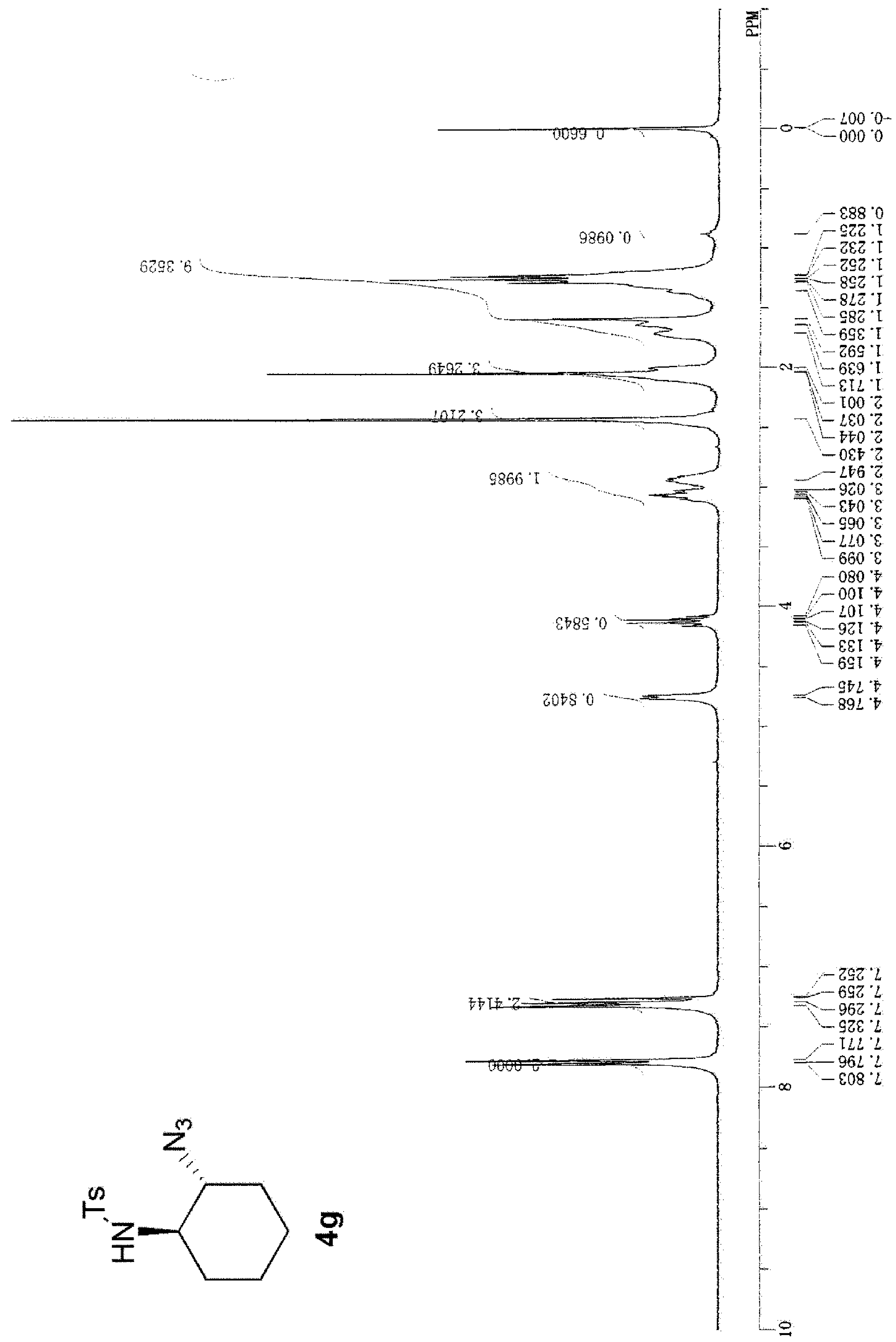

S48 


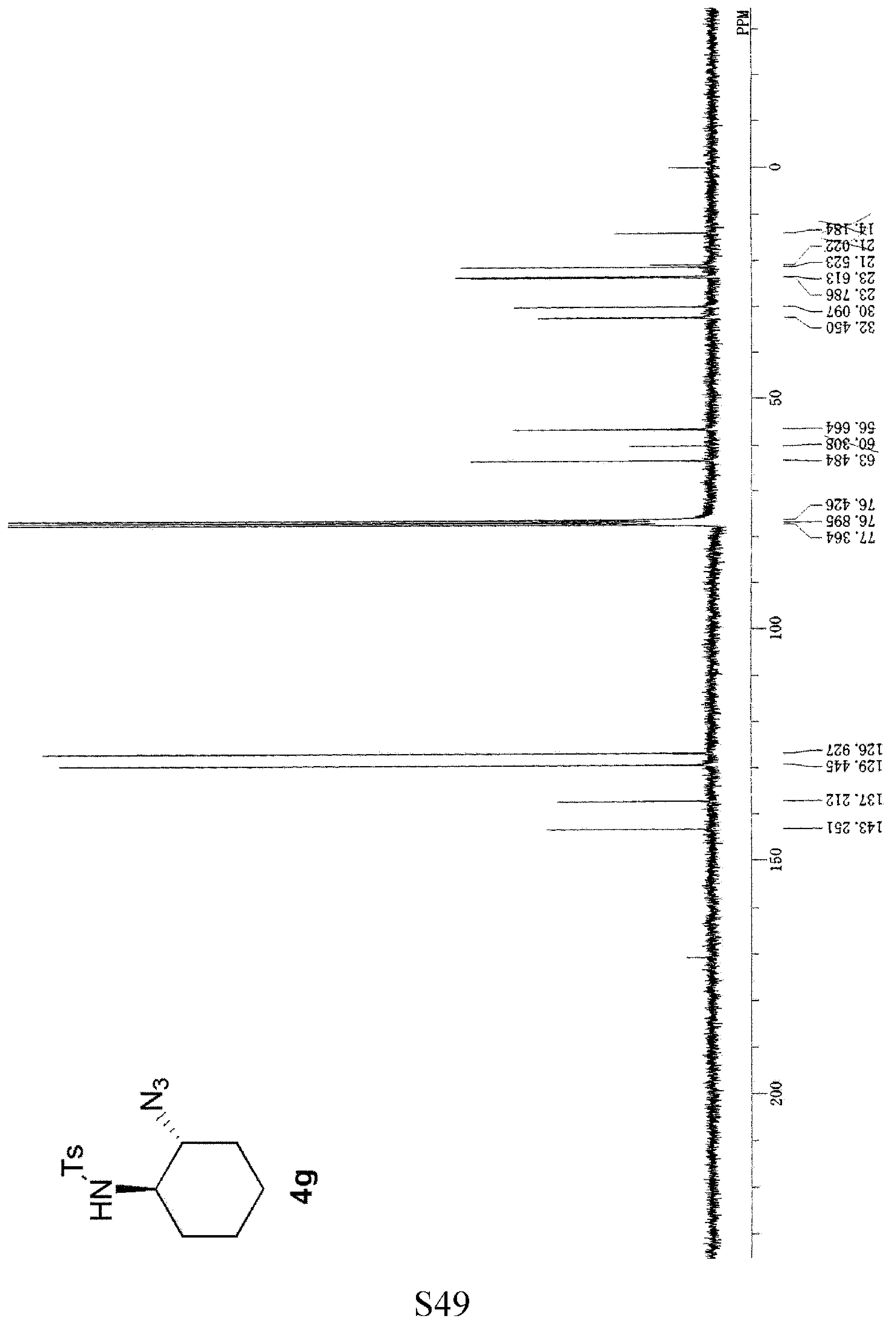




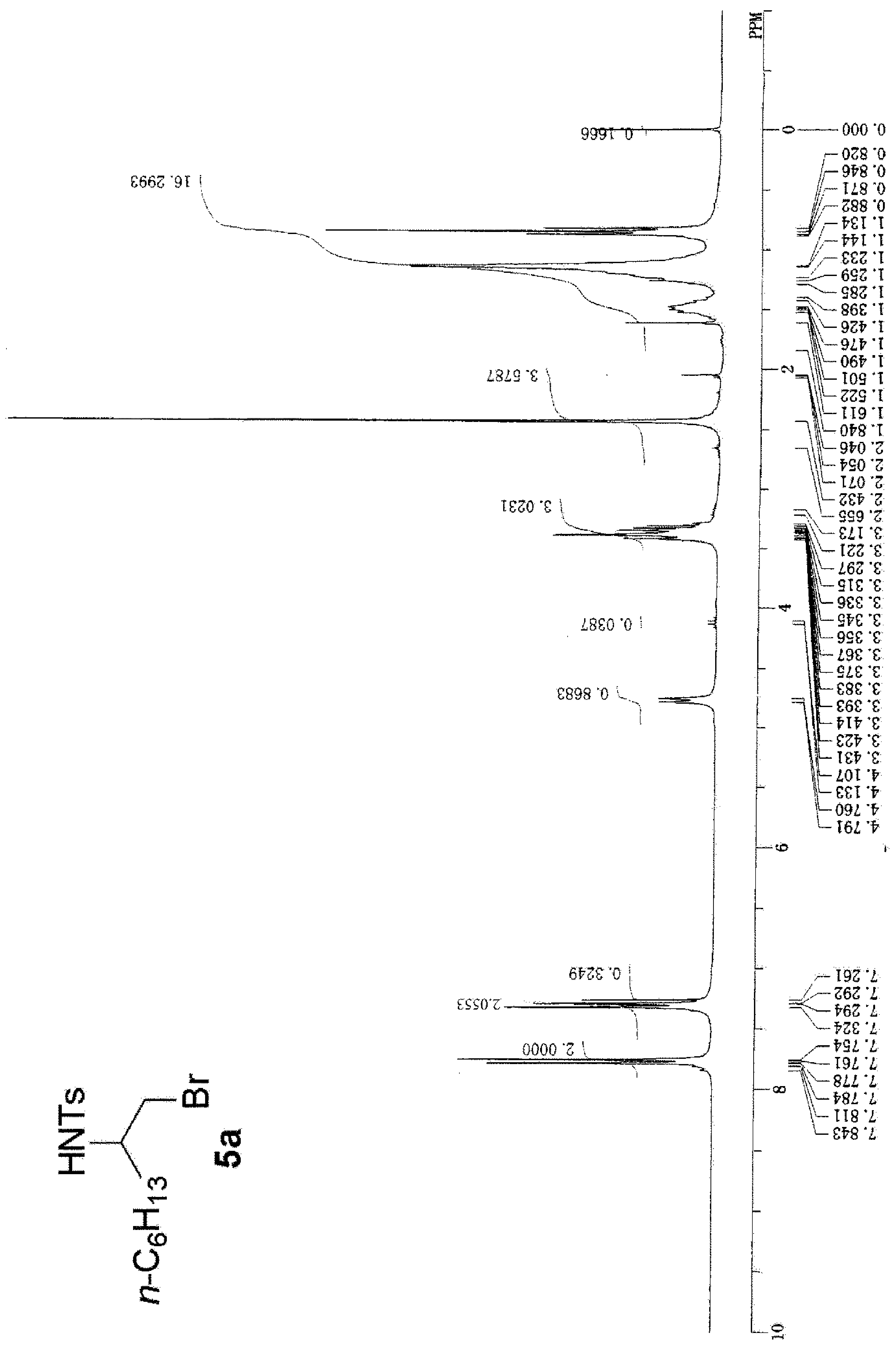




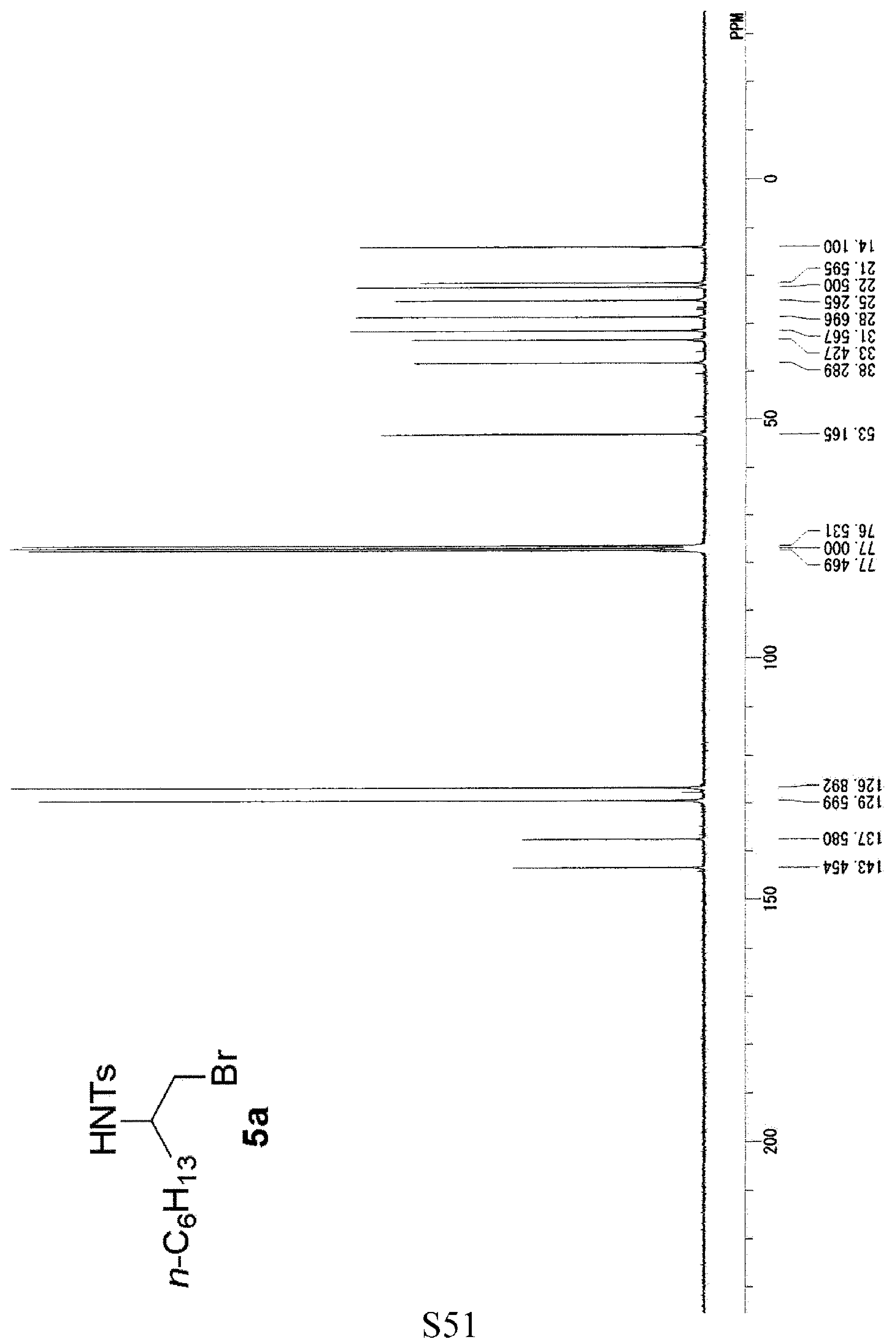




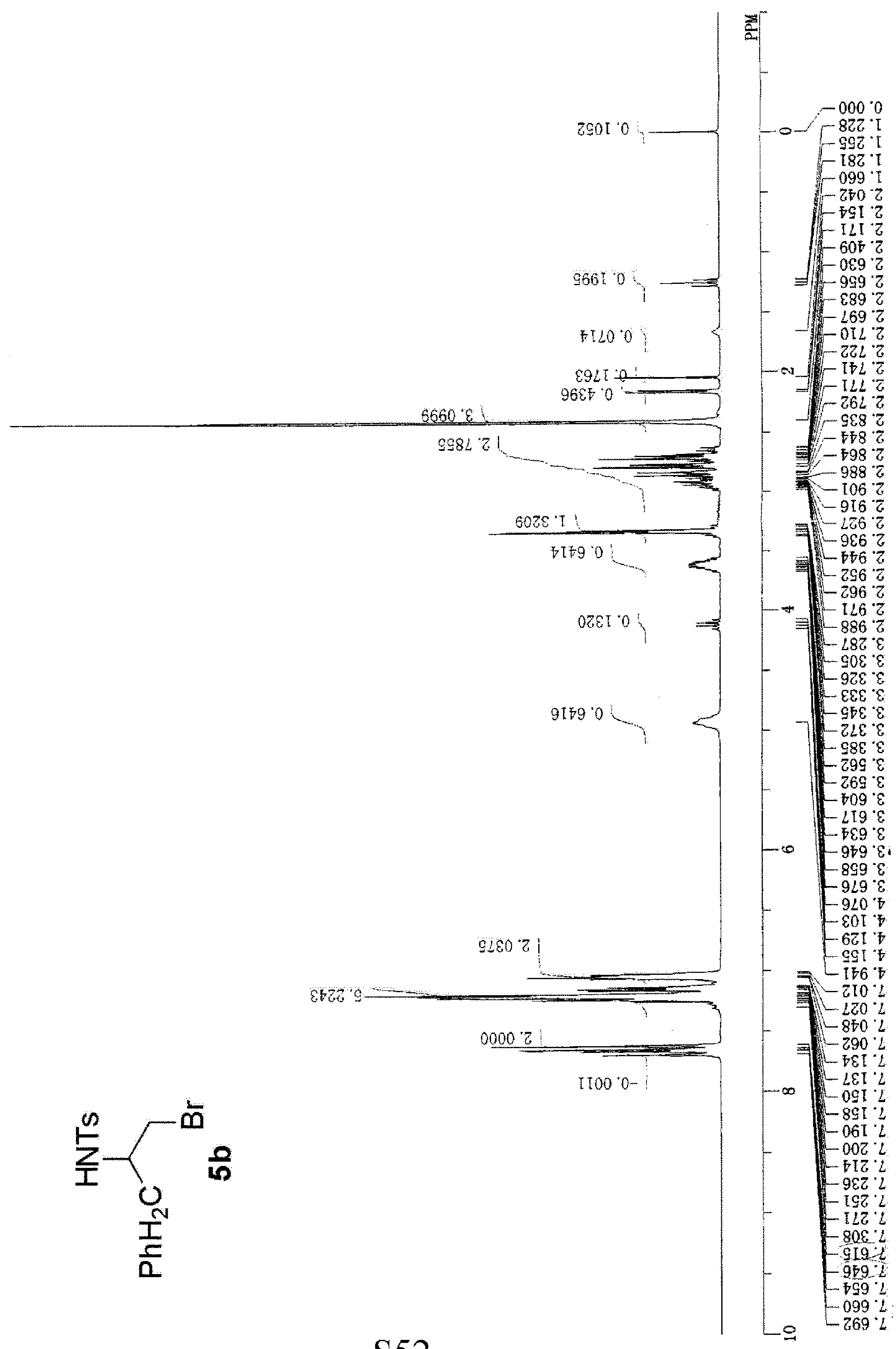

S52 


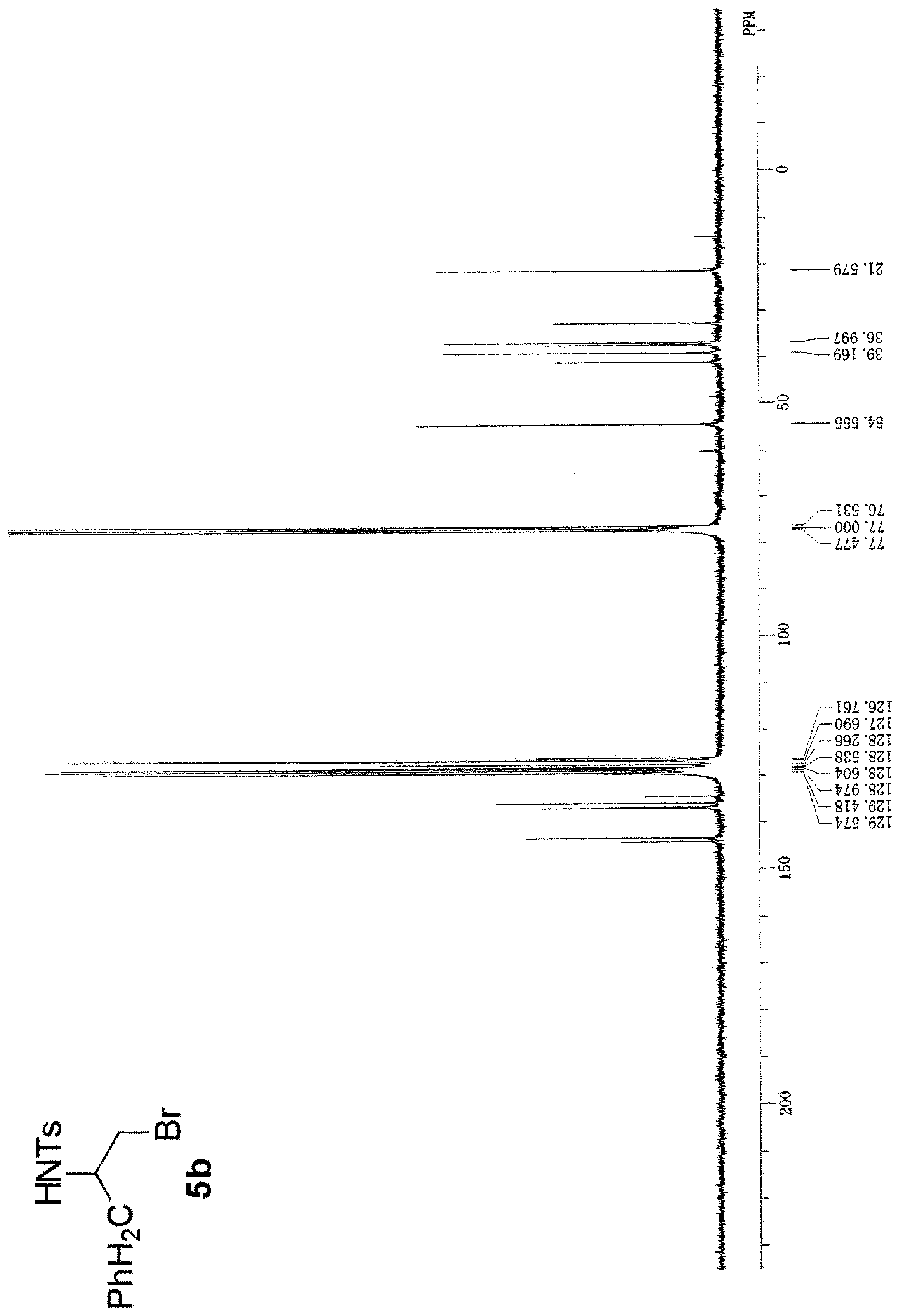

S53 


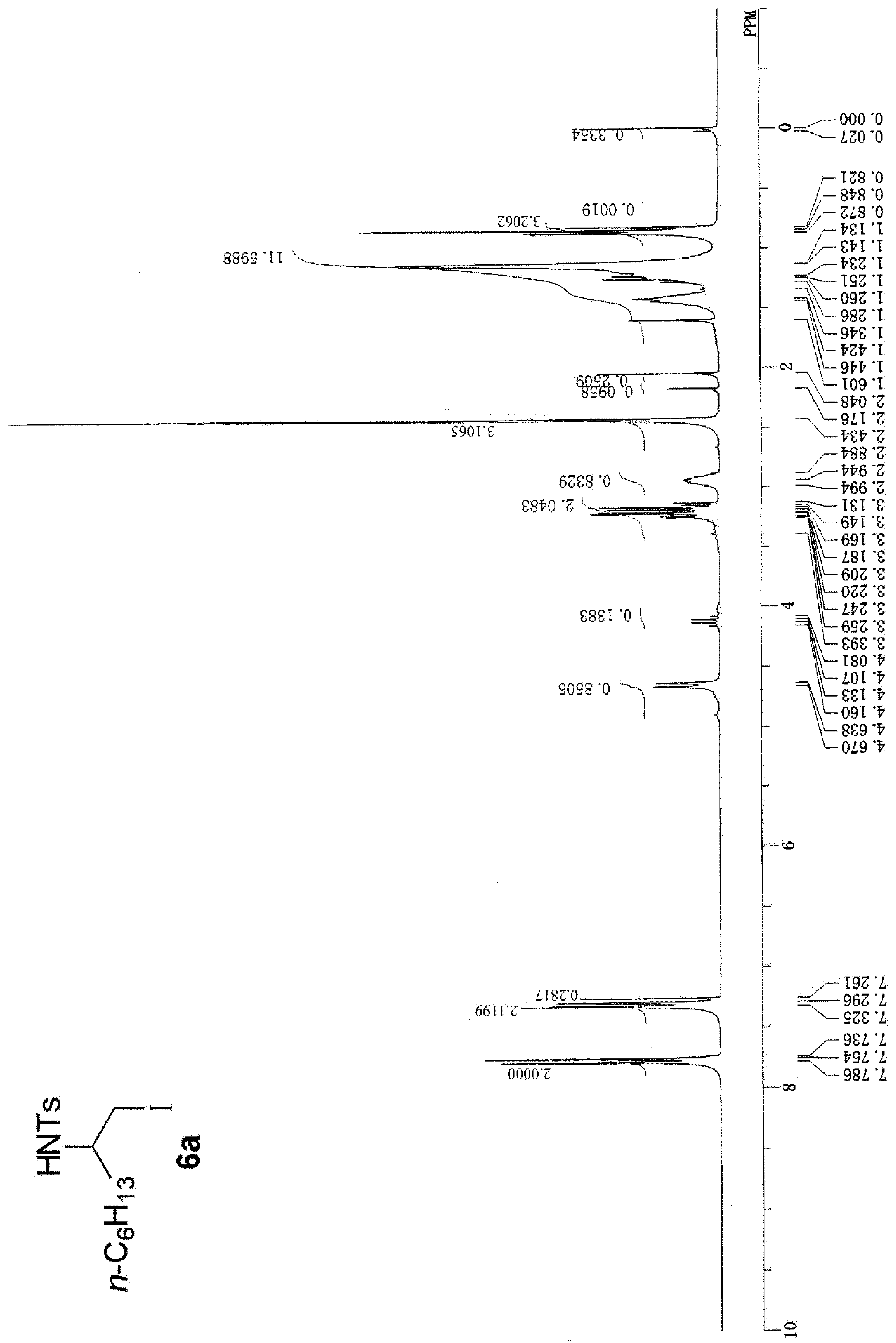

S54 


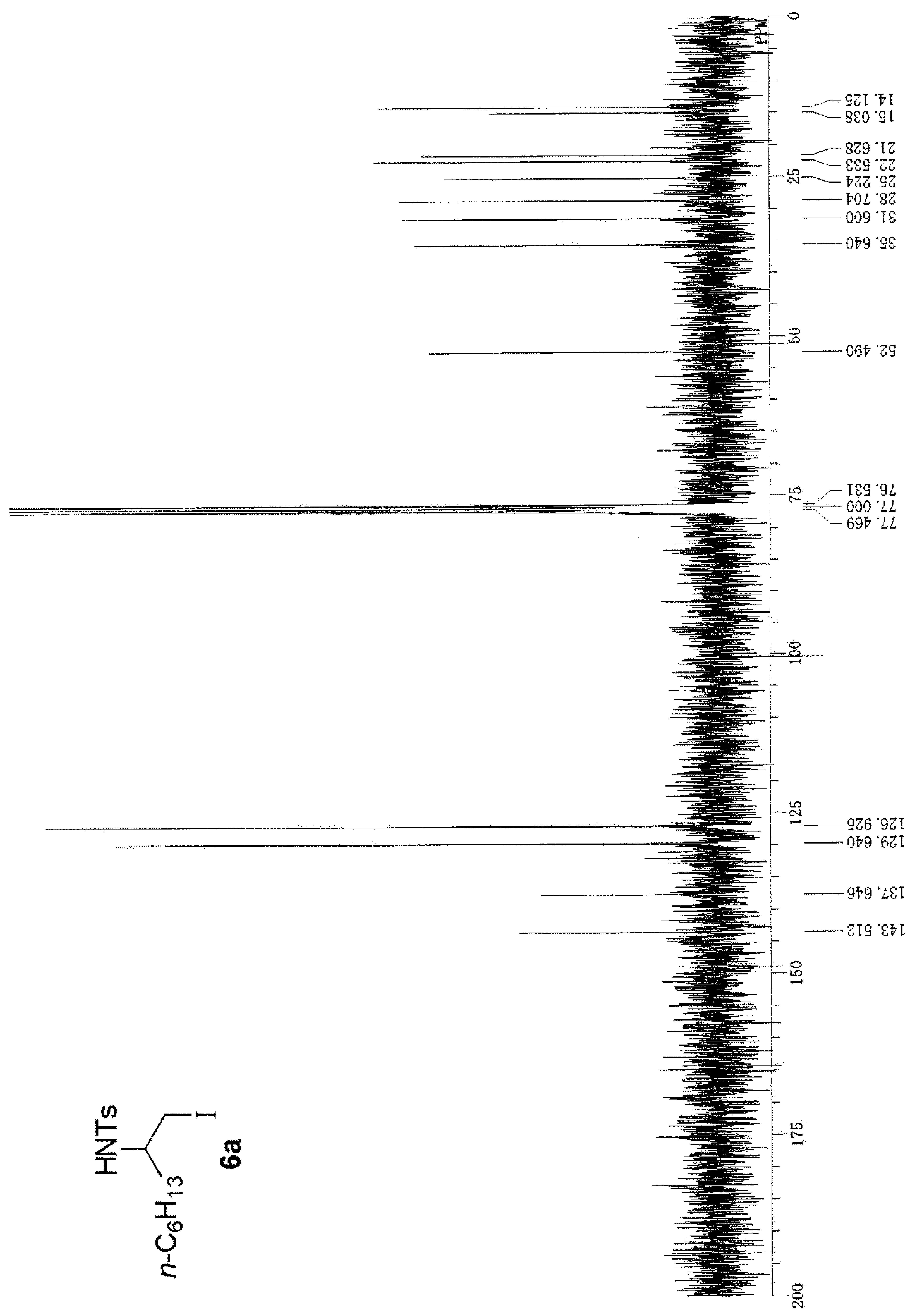




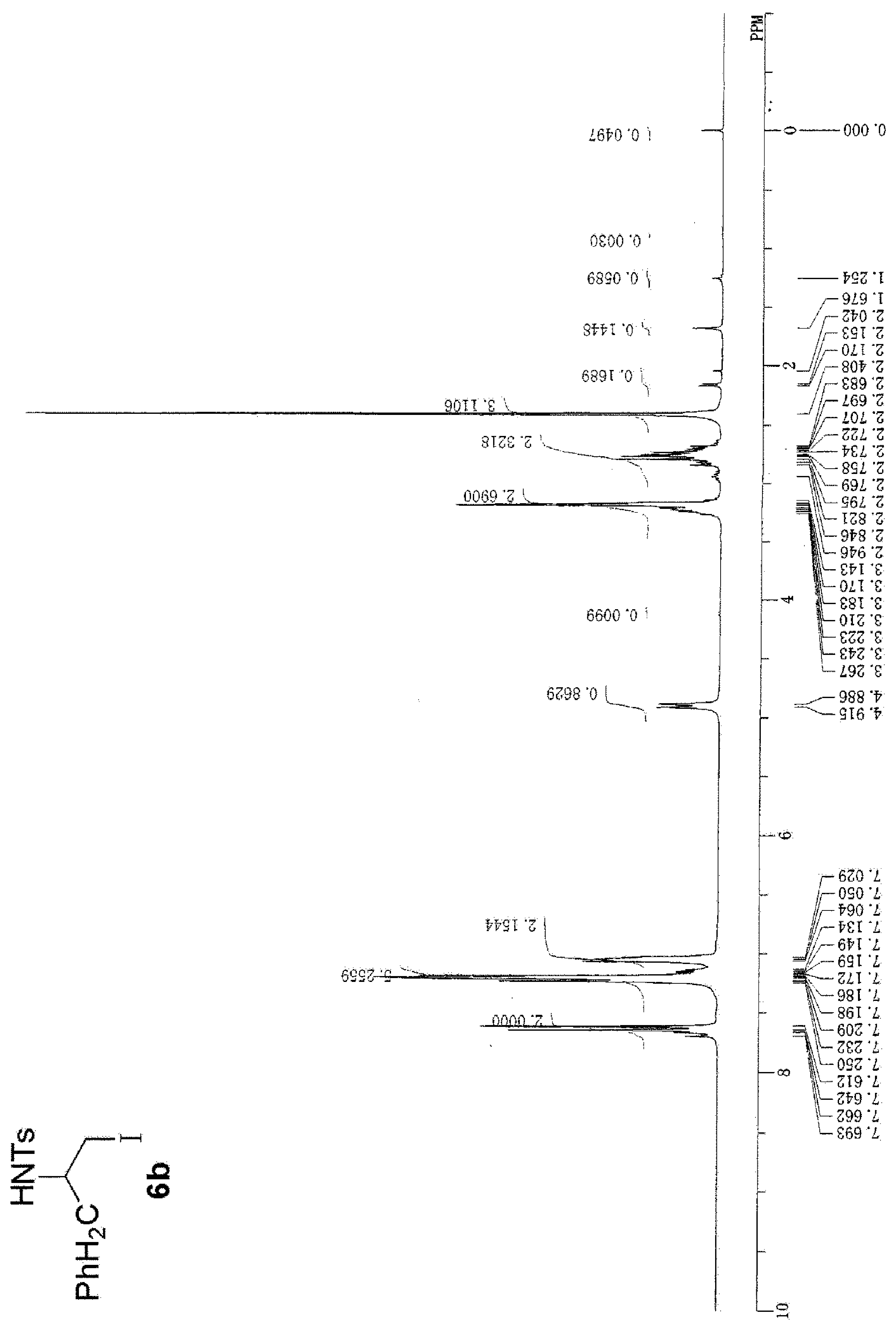




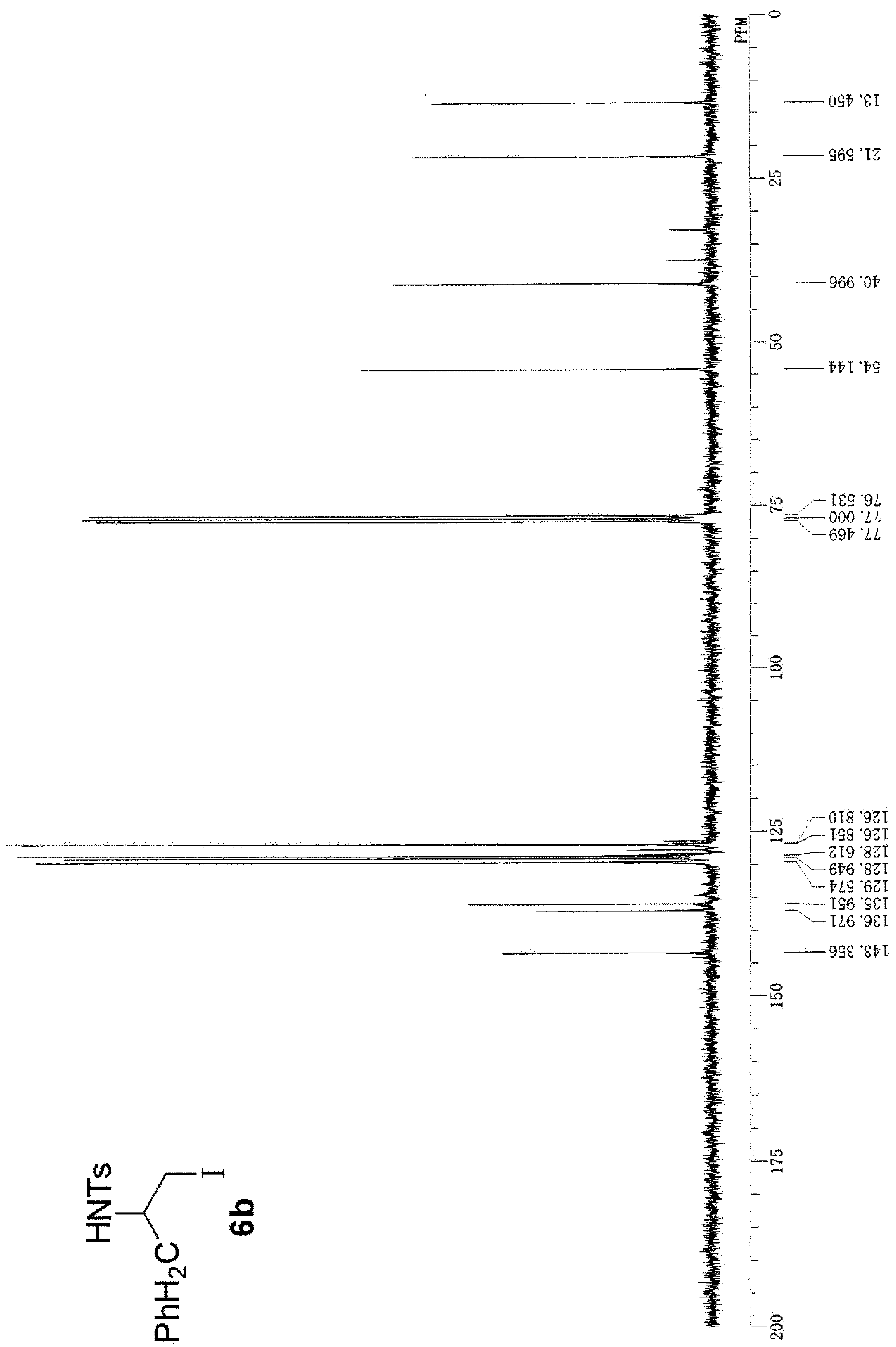

